
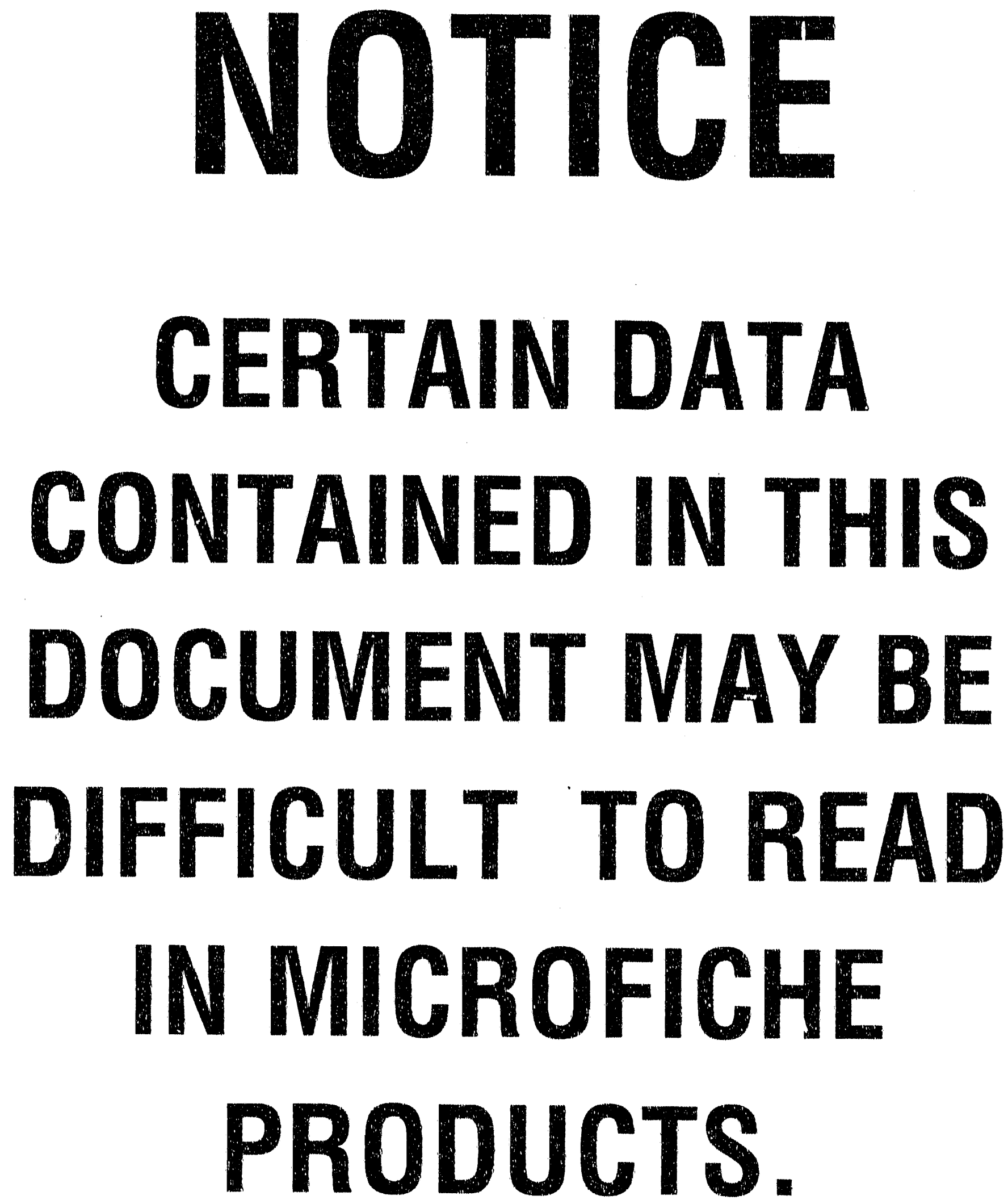


\title{
ENHANCING THE USE OF COALS BY GAS REBURNING-SORBENT INJECTION
}

\author{
DE-FG 22.87PC 79796
}

Environmental Monitoring Report

Quarterly Report No. 8

For the Period

April 1 - June 30, 1992

\author{
Prepared for \\ U.S. Department of Energy \\ Gas Research Institute \\ State of Illinois Department of \\ Energy and Natural Resources
}

\author{
Prepared by \\ Energy and Environmental Research Corporation \\ 18 Mason \\ Irvine, California 92718
}

July 27,1992

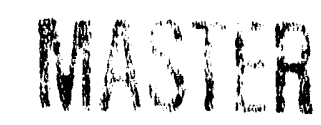




\section{$\therefore \quad$}

TABILE OF CONTENTS

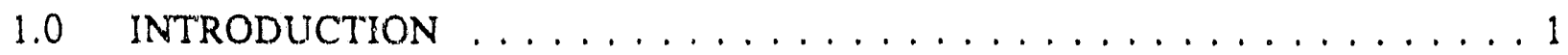

2.0 ENVIRONMENTAL MONTTORING $\ldots \ldots \ldots \ldots \ldots \ldots \ldots \ldots \ldots$

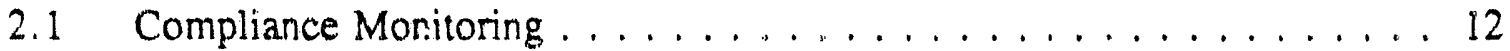

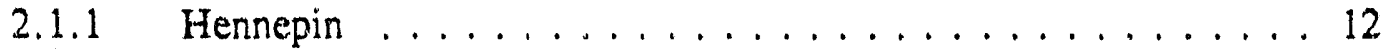

2.1 .2 Lakeside $\ldots \ldots \ldots \ldots \ldots \ldots \ldots \ldots \ldots \ldots \ldots \ldots$

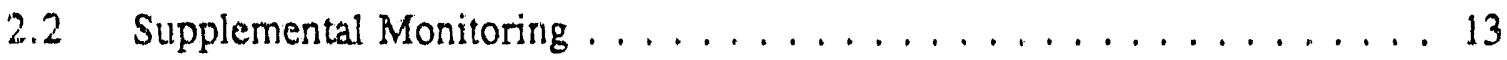

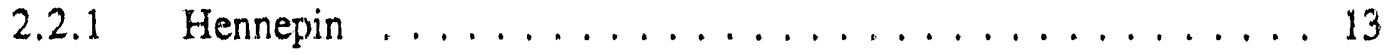

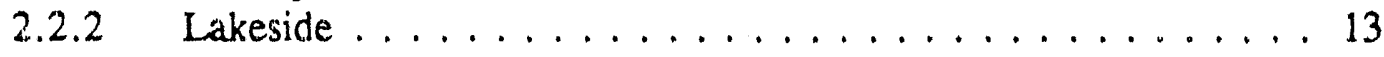

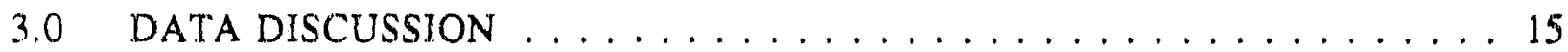

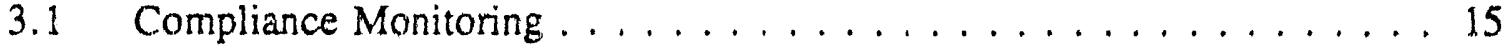

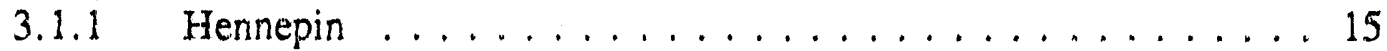

3.1 .2 Lakeside . . . . . . . . . . . . . . . . . . . 15

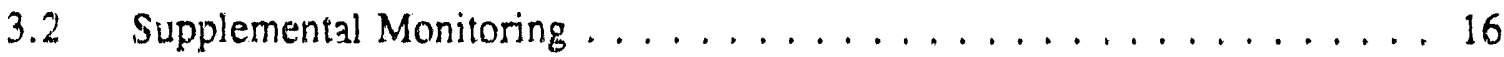

3.2.1 Hennepin ........................ 16

3.2 .2 Lakeside $\ldots \ldots \ldots \ldots \ldots \ldots \ldots \ldots \ldots \ldots$

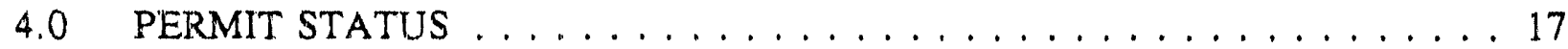

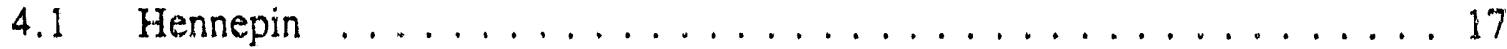

4.2 Lakeside $\ldots \ldots \ldots \ldots \ldots \ldots \ldots \ldots \ldots \ldots \ldots \ldots$

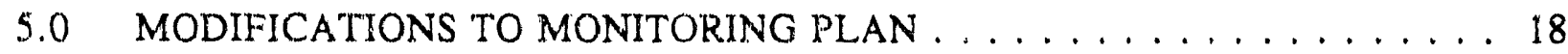

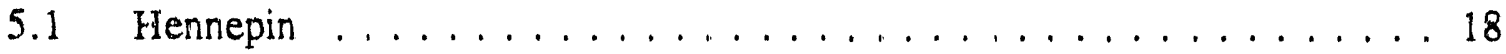

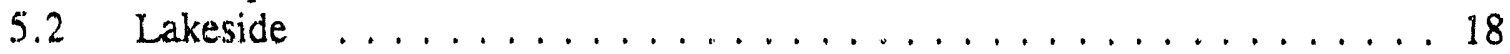

Table 1 Hennepin Project Monitoring in Phases $I$ and II . . . . . . . . . . 4

Table 2 Hennepin Project Monitoring in Phase IIx . . . . . . . . . . . . . . 5

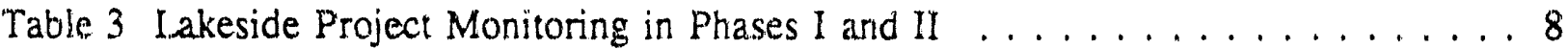

Table 4 Lakeside Project Monitoring in Phase III . . . . . . . . . . . . . . . 10

Table 5 Continuous Emission Monitoring Data for Gaseous Emissions . . . . . . . . 14

APPENDIX A: Hennepin Discharge Monitoring and Coal Analysis Reports

APPENDIX B: Lakeside Discharge Monitoring and Excess Opacity Reports

APPENDIX C: Hennepin Draft NPDES Permit 


\subsection{INTRODUCTION}

Clean Coal Technology implies the use of coal in an environmentally acceptable manner. Coal combustion results in the emission of two types of acid rain precursors: oxides of sulfur $\left(\mathrm{SO}_{\mathrm{x}}\right)$ and oxides of nitrogen $\left(\mathrm{NO}_{\mathrm{x}}\right)$. This Clean Coal Technology project will demonstrate a combination of two developed technologies to reduce both $\mathrm{NO}_{\mathrm{x}}$ and $\mathrm{SO}_{\mathrm{x}}$ emissions: gas reburning an I calcium based dry sorbent injection. The demonstrations will be conducted on two pre-NSPS utility boilers representative of the U.S. boilers which contribute significantly to the inventory of acid rain precursor emissions: tangentially and cyclone fired units. Because of cost growth and lack of available funding, no further work has been done after Phase I at site $B$; the wall fired unit.

Gas reburning is a combustion modification technique that consists of firing 80-85 percent of the fuel (corresponding to the total heat release) in the lower furnace. Reduction of $\mathrm{NO}_{\mathrm{x}}$ to molecular nitrogen $\left(\mathrm{N}_{2}\right)$ is accomplished via the downstream injection of the remaining fuel requirement in the form of natural gas (which also reduces the total $\mathrm{SO}_{\mathrm{x}}$ emissions). In a third stage, bumout air is injected at lower temperatures in the upper furnace to complete the combustion process without generating significant additional $\mathrm{NO}_{\mathrm{x}}$.

Dry sorbent injection consists of injecting calcium based sorbents (such as limestone, dolomite, or hydrated lime) into the combustion products. For sulfation of the sorbent to $\mathrm{CaSO}_{4}$, an injection temperature of about $1230^{\circ} \mathrm{C}$ is optimum, but calcium-sulfur reactions can also take place at lower temperatures. Thus, the sorbent may be injected at different locations, such as with the burnout air, at the exit from the superheater, or into the ducting downstream of the air heater with $\mathrm{H}_{2} \mathrm{O}$ added for humidification. The calcium sulfate or sulfite products are collected together with unreacted sorbent and fly ash by the electrostatic precipitator. The specific goal of this project is to demonstrate $\mathrm{NO}_{\mathrm{X}}$ and $\mathrm{SO}_{\mathrm{x}}$ emission reductions of 60 percent and 50 percent, respectively, on iwo coal fired utility boilers having the design characteristics mentioned above. 
Ast the inception of the project, Host Site Agreements were signed by EER and three utility companies in the State of Illinois: Site A, Illinois Power Company (Hennepin Unit 1, 71MW (net) tangentially fired boiler in Hennepin); Site B, Central Illinois Light Company (Edwards Unit 1, $117 \mathrm{MW}$ (net) front wall fired boiler in Bartonville); and Site C, City Water Light and Power (Lakeside Unit 7, $33 \mathrm{MW}$ (net) cyclone fired boiler in Springfield).

Funding for this project is provided by the Department of Energy (DOE), the Gas Research Institute (GRI), and the State of Illinois Department of Energy and Natural Resources (ENR)--the other funding participants. GRI and ENR are responsible for funding approximately one-third and one-sixth, respectively, of the total project costs

To achieve the objectives of the project, it is conducted in the following three phases at each site:

Phase 1: Design and Permitting

Phase II: Construction and Startup

Phase III: Operation, Data Collection, Reporting and Disposition

Phase I work has been completed for all three sites. Because of cost growth and lack of available funding, no further work has been done after Phase I at Site $B$, the wall fired unit. At Site A, Phase II has been completed and Phase III long-term tests are currently underway. Site $\mathrm{C}$ is currently in Phase II of the program.

In accordance with the cooperative agreement, EER has prepared an Environmental Monitoring Plan to verify that no adverse environmental impacts would be associated with the project, to determine if projected emission control levels are being met, and to maintain a data base for future development of GR-SI technology. Tables 1 through 4 show the environmental monitoring to be conducted at Sites A and C, (Hennepin and Lakeside) during Phases I, II and III of the project. 
The following section provides a description of the monitoring which has been conducted during the previous quarter at the host sites. A discussion of the monitoring results is presented in Section 3. Section 4 provides a status report of permits obtained for the project at each facility, and Section 5 discusses any proposed changes in the original monitoring plan based on available information. 


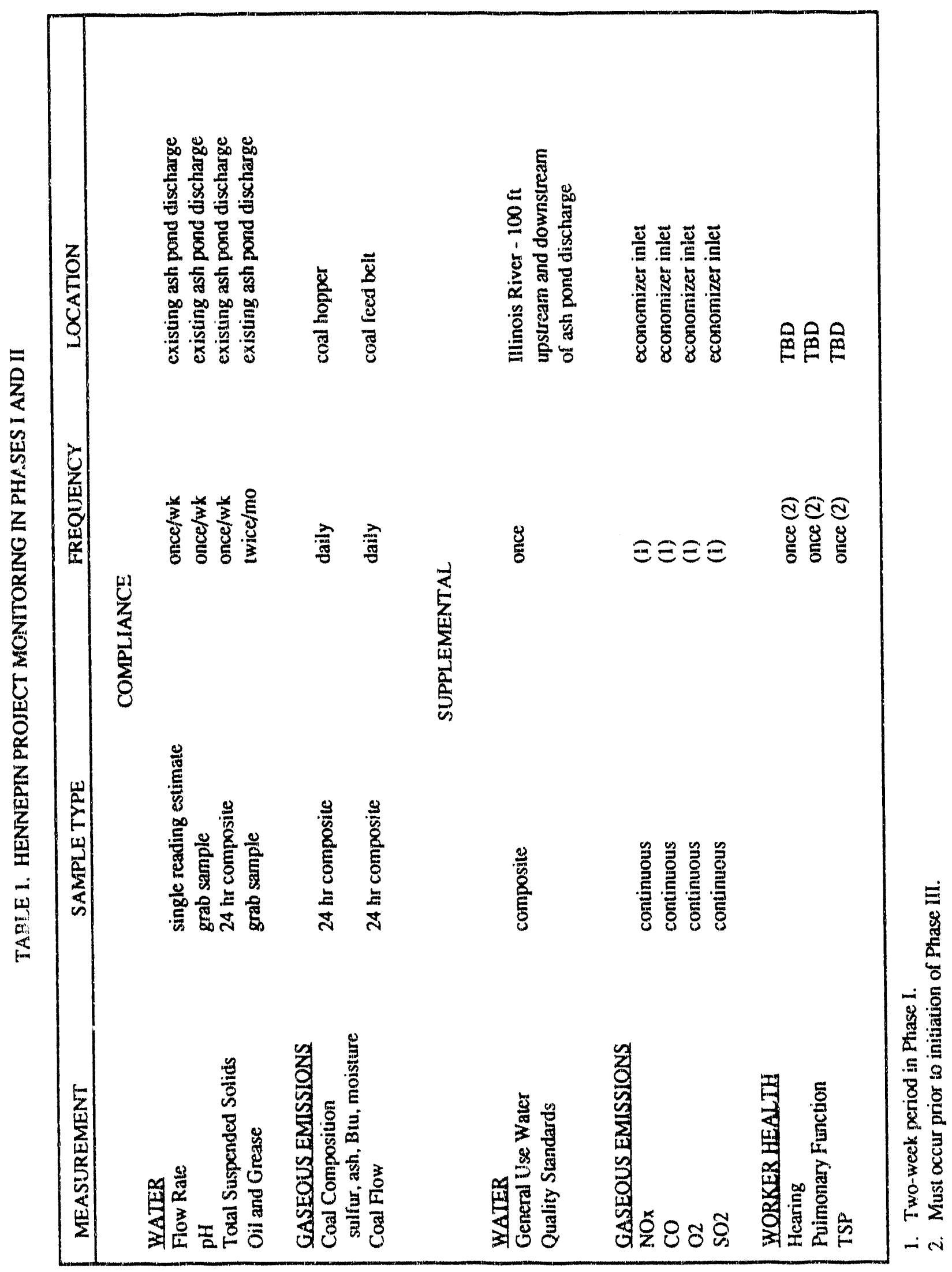




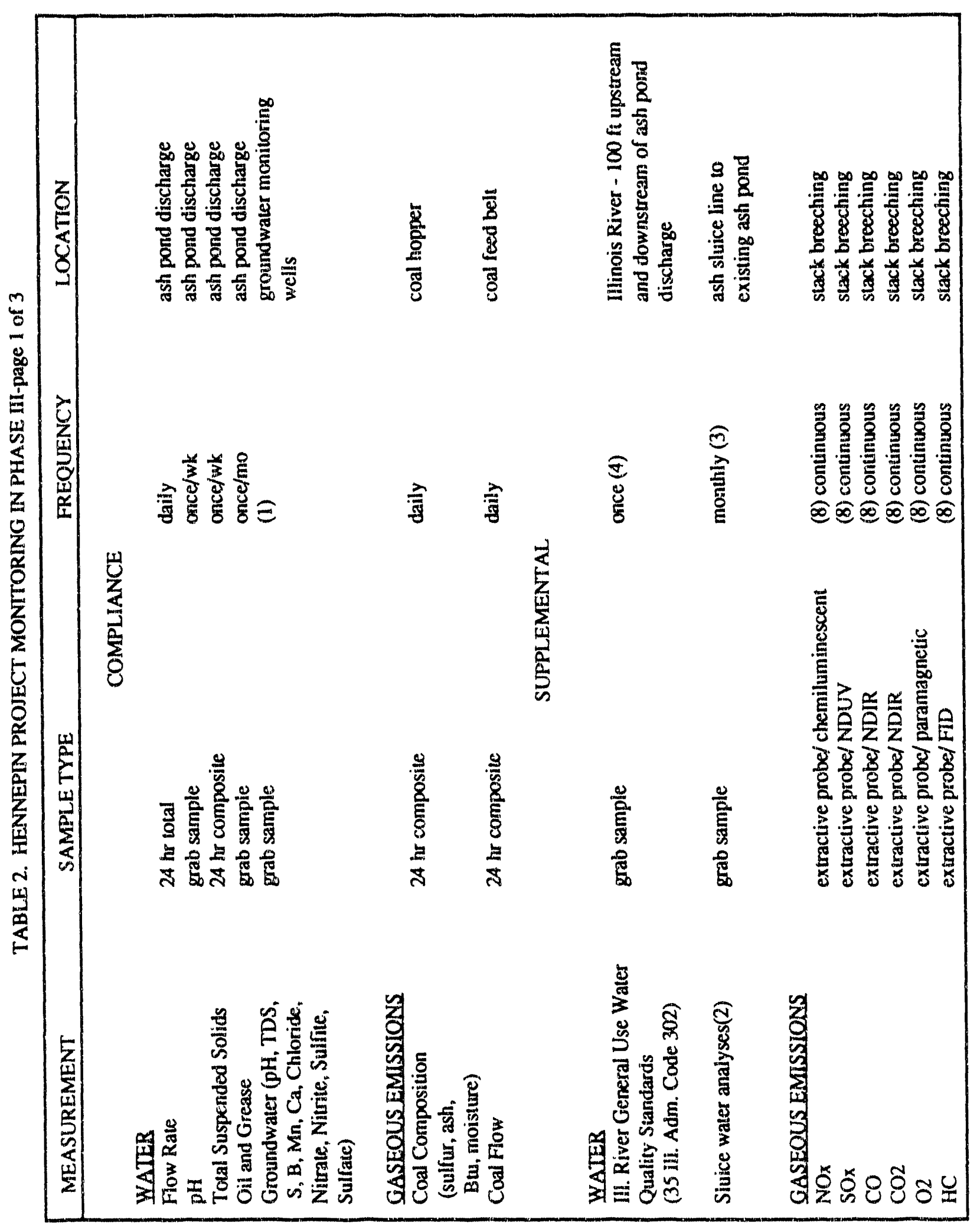




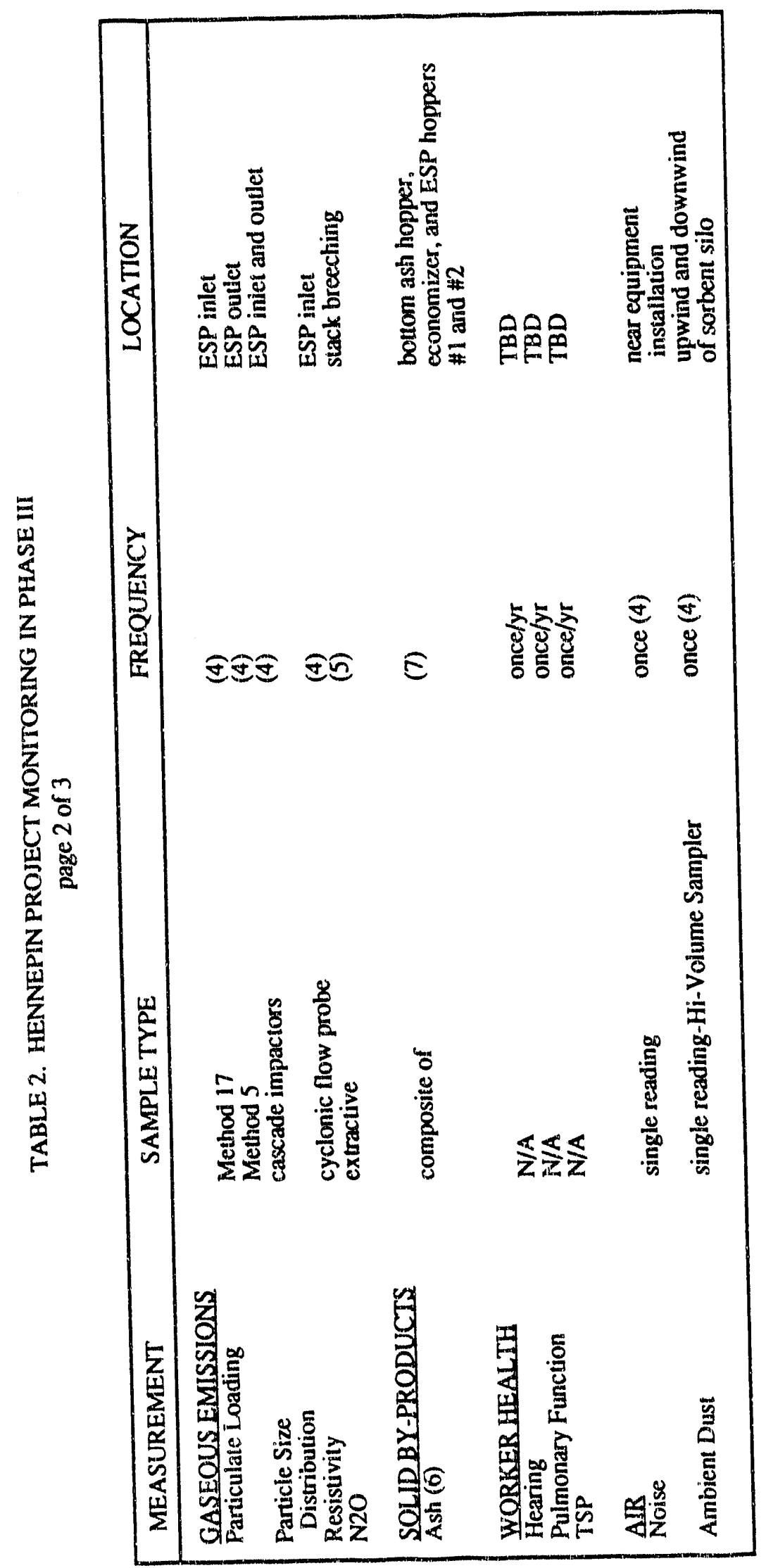




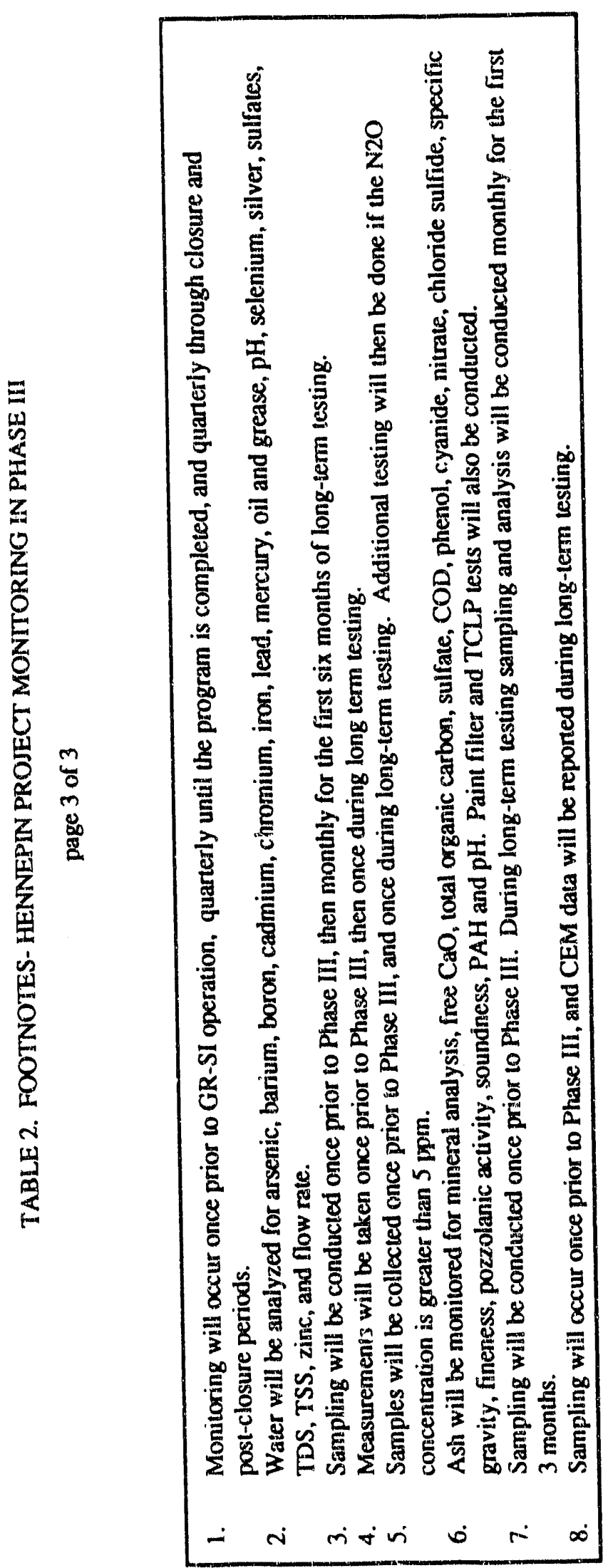




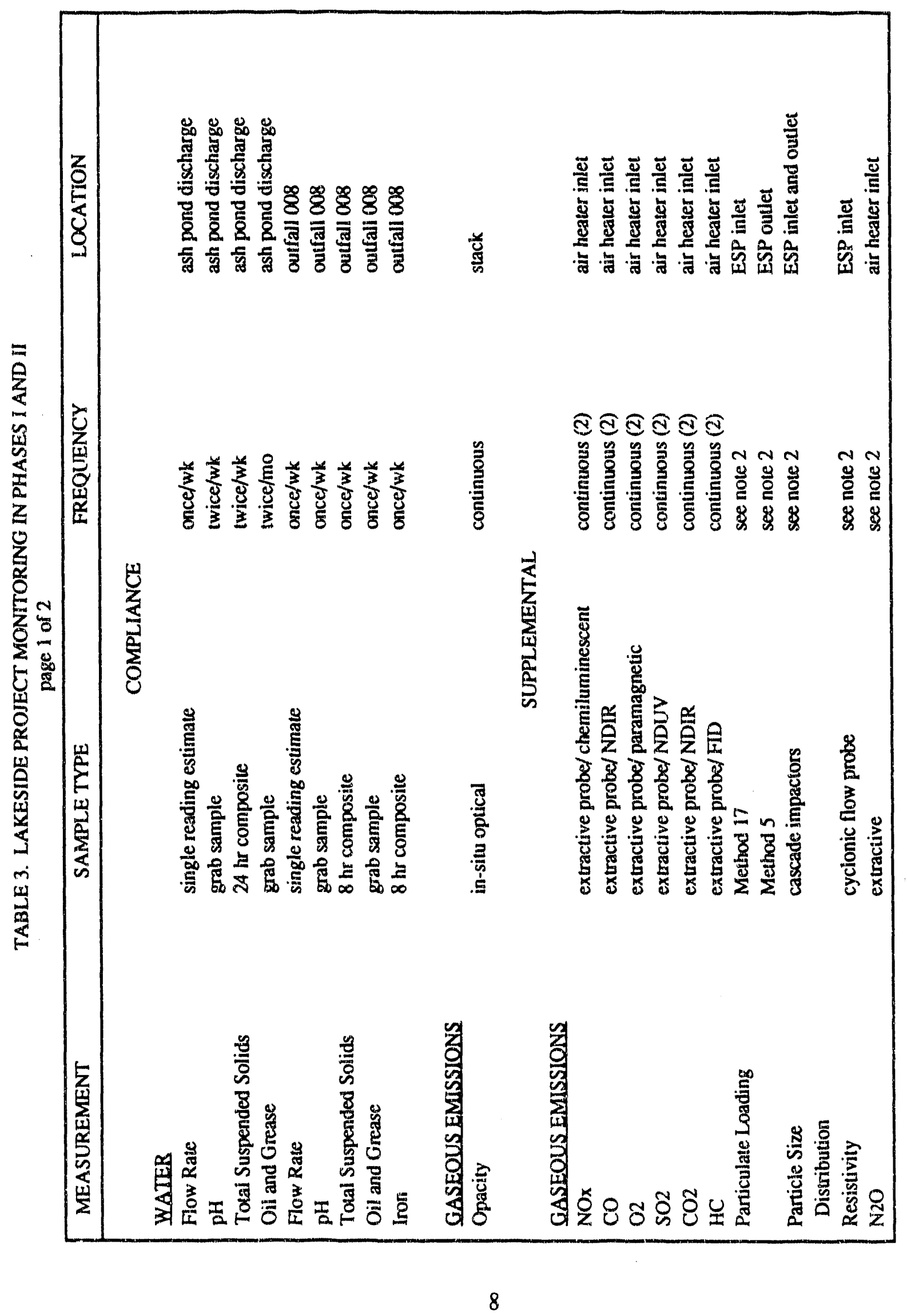




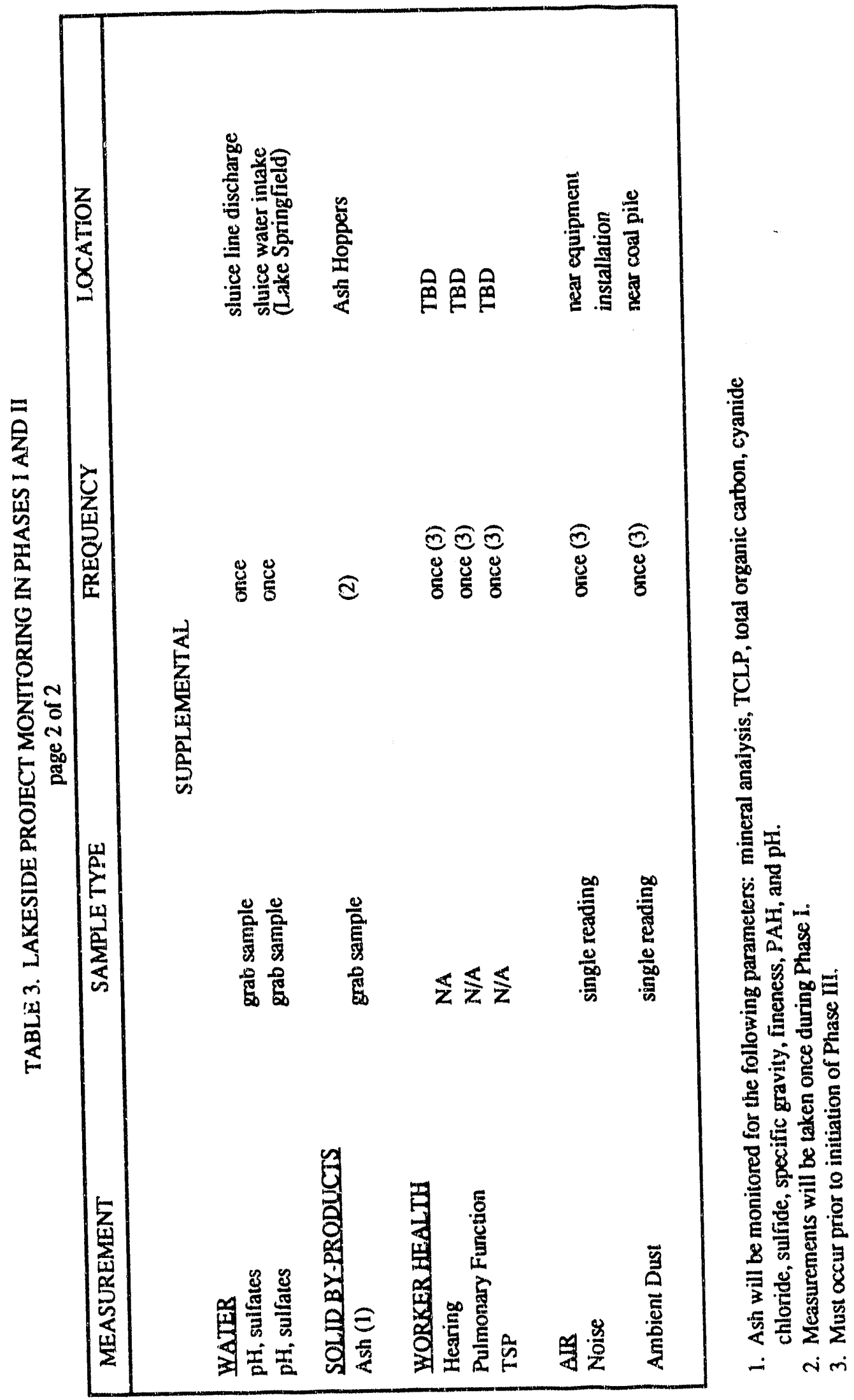




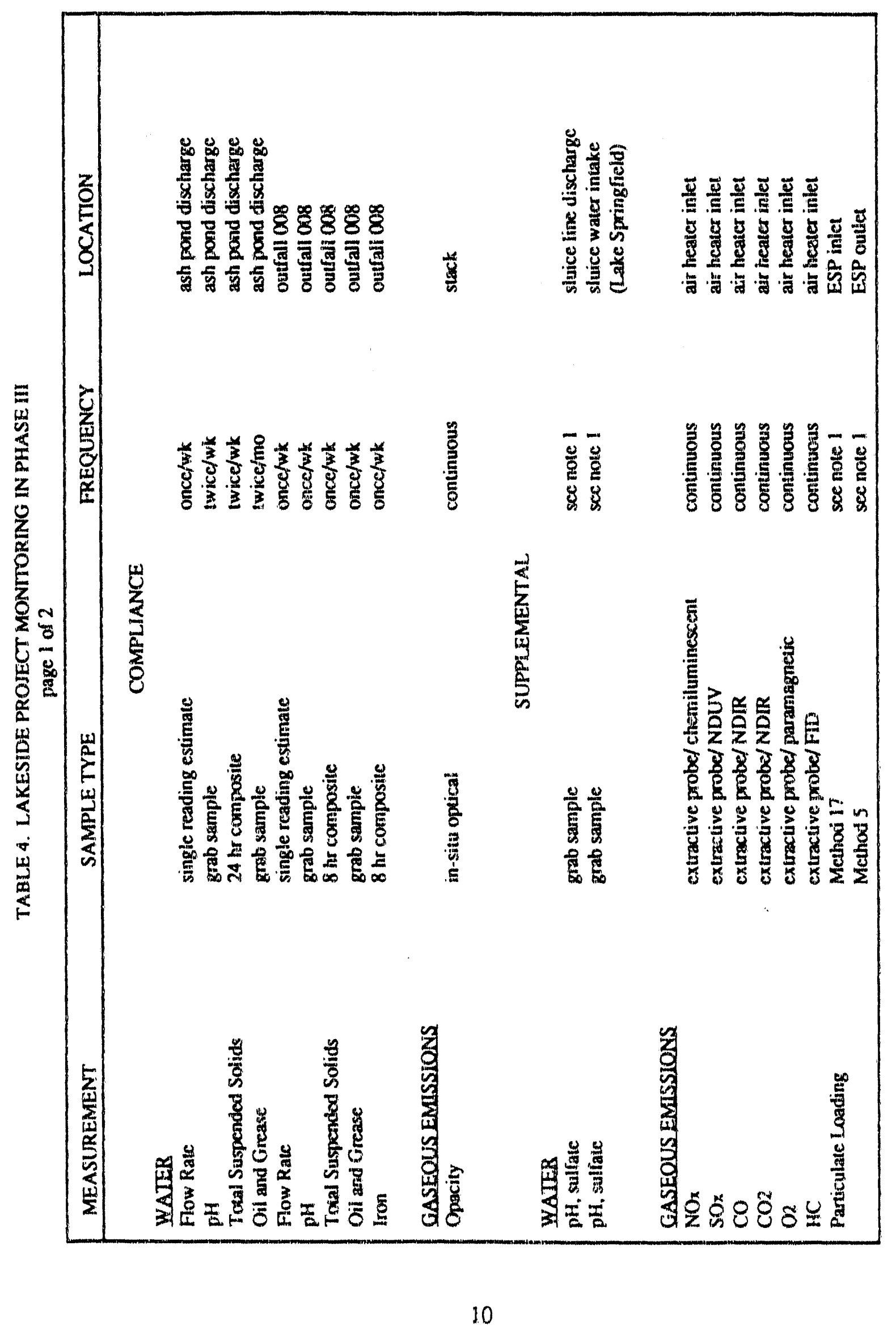




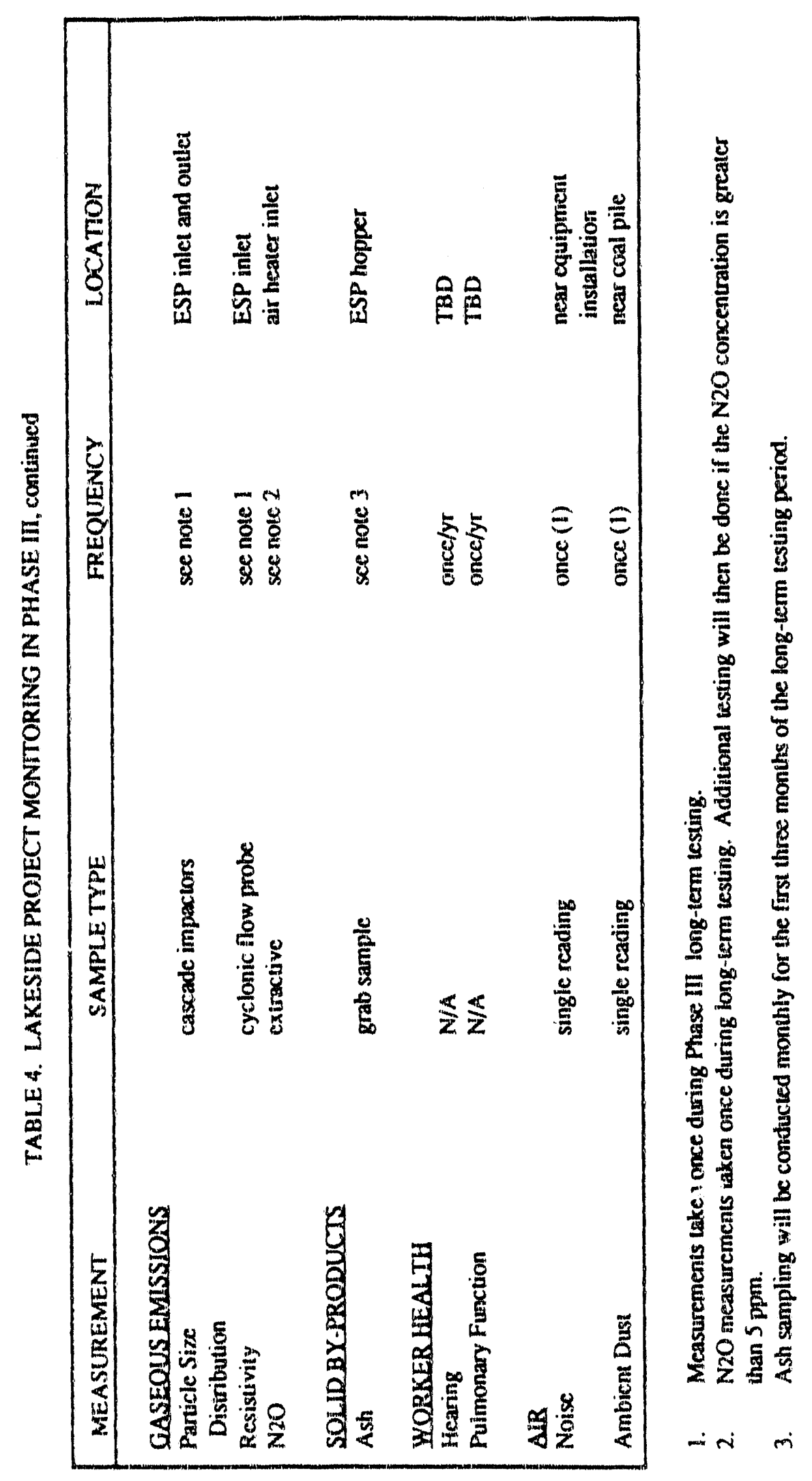




\subsection{ENVIRONMENTAL MONITORING}

Both compliance and supplemental monitoring was conducted at the Hennepin site during the previous quarter. At the Lakeside site, only compliance monitoring was conducted during the previous quarter. The monitoring results from Hennepin and Lakeside are presented in the following sections.

\section{$2.1 \quad$ Compliance Monitoring}

Compliance monitoring is that monitoring required by Federal, State or local authorities.

\subsubsection{Hennepin}

The compliance monitoring conducted at Hernepin during the quarter which directly influences this project includes NPDES permit monitoring, coal quality reports, and groundwater monitoring data. Illinois Power submits NPDES Discharge Monitoring Reports to the lllinois EPA, on a monthly basis. Coal quality reports are submitted to Illinois EPA on a quarterly basis. Copies of the discharge monitoring and coal quality reports for April, May, and June are included in Appendix $A$ of this report. Groundwater monitoring results for this quarter were unavailable; they will be reported in the October, 1992 quarterly report.

\section{$2.1 .2 \quad$ Lakeside}

The compliance monitoring conducted at Lakeside during the quarter which directly influences this project includes NPDES permit monitoring and gaseous emissions opacity reports. Copies of the NPDES and excess opacity reports for April, May, and June are included in Appendix $B$ of this report. 


\section{$2.2 \quad$ Supplemental Monitoring}

2.2.1 Hennepin

The supplemental monitoring reported during this quarter consisted only of Continuous Emission Monitor daily averages for $\mathrm{SO}_{2}, \mathrm{NO}_{\mathrm{x}}, \mathrm{CO}_{2}, \mathrm{CO}, \mathrm{O}_{2}$, and $\mathrm{HC}$. The results of the continuous emission monitoring are outlined in Table 5.

\subsubsection{Lakeside}

No supplemental monitoring was conducted at Lakeside during this quarter. 


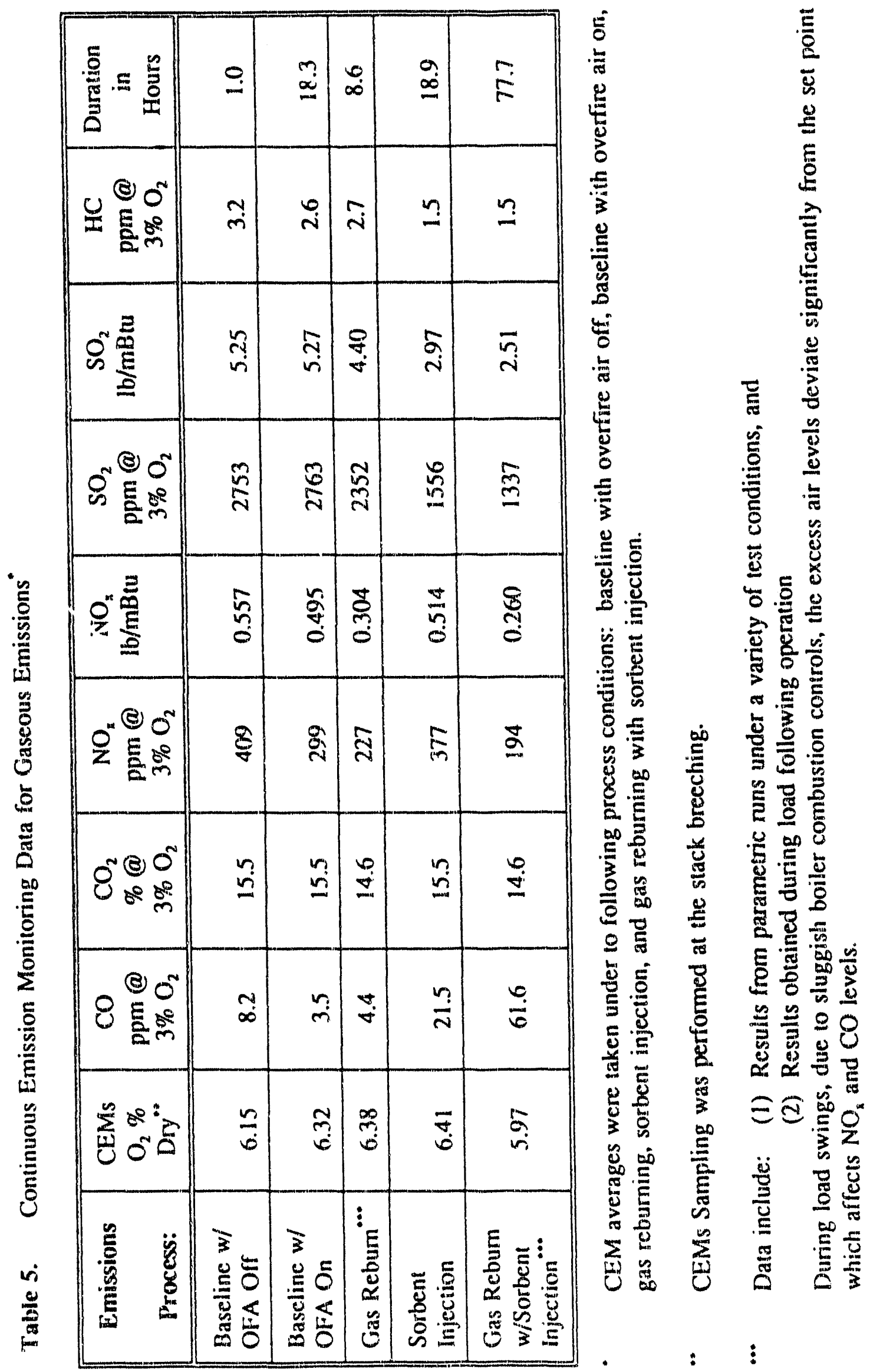




\section{$3.1 \quad$ Compliance Monitoring}

\subsubsection{Hernepin}

The discharge monitoring reports for April through June indicated that no effluents were discharged from the ash pond to the Illinois River. The gaseous emissions for the coal composition did not exceed the regulatory emission rate of $17,050 \mathrm{lbs}$. of $\mathrm{SO}_{2}$ per hour. The average $\mathrm{lbs}$. of $\mathrm{SO}_{2}$ per MBTU for this quarter was 5.30, based on the coal analysis. The GRSI demonstration program substantially reduced the $\mathrm{SO}_{2}$ emissions during the process operational hours.

\subsubsection{Lakeside}

The discharge monitoring reports for the ash pond outfall structure and the coal pile runoff during the months of April through June indicate that the ash pond outfall did not exceed the NPDES permit limitations. The coal pile runoff did not produce a discharge stream during this quarter.

The gaseous emissions opacity reports for April through June indicate that the opacity limit of 30 percent was exceeded during each of the three months. April exceedances were due startup, shutdown, precipitator failure, maintenance repairs, or unknown causes. Excess opacity readings in May were attributed to startup, shutdown, unit trip, precipitator field trip, air damper malfunction, and switching precipitator fields. June excess opacity measurements were due to startup, shutdown, precipitator malfunction, or unknown causes. 


\section{$3.2 \quad$ Supplemental Monitoring}

\subsubsection{Hennepin}

Continuous Emission Monitoring averages were taken under the following process conditions: baseline with over fire air off; baseline with over fire air on; gas reburning, sorbent injection, gas reburning with sorbent injection. These averages show that there was a 53 percent reduction in $\mathrm{NO}_{\mathrm{x}}$ from baseline with overfire air off, when implementing gas reburning with sorbent injection. In addition, there was a 51 percent reduction in $\mathrm{SO}_{2}$ from baseline with overfire air off, when implementing gas reburning with sorbent injection.

\subsubsection{Lakeside}

No supplemental monitoring was conducted at Lakeside during this quarter. 
Permits have been obtained for construction and operation of the GR-SI system at Hennepin. At Lakeside, construction is in progress, and operating permits have not been obtained yet. The following sections describe the permits obtained at each host site.

\section{1 $\quad$ Hennepin}

IP applied for a construction permit for the GR-SI project at Hennepin on July 7, 1989, and Illinois Environmental Protection Agency granted that permit on October 18, 1989. A modification to the construction permit for operation of the GR-SI demonstration for a period of 270 days was requested. This permit was granted June 21, 1990. A permit also was required to install the $\mathrm{pH}$ adjustment system for the GR-SI ash transport water to the existing ash pond. This permit was granted on July 25, 1990. Negotiations between Illinois Power, the Illinois EPA, and the U.S. EPA has resulted in the issuance of a revised draft Hennepin NPDES permit. During August, compliance monitoring will be performed on the unit's air emissions per requirements of the draft permit. A copy of this draft permit is included in Appendix $\mathrm{C}$ of this report.

\subsection{Lakeside}

On January 8, 1990, CWLP submitted a PSD permit application for the GR-SI demonstration at Lakeside Unit 7. On July 5, 1990, CWLP granted the Illinois Environmental Protection Agency a waiver from its statutory deadline to review the permit application (180 days) for a period of 120 days. Permit approval was received November 16, 1990. EER is in the process of applying for a Special Waste Permit through the Christian County Landfill for the future disposal of the Lakeside fly ash waste stream. A pre-permit for the disposal of special waste at the Christian County Landfill was obtained on March 18, 1992. 
5.1

Hennepin

There are no recommended modifications to the proposed monitoring plan as outlined in Table 1 and 2.

5.2 Lakeside

There are no recommended modifications to the proposed monitoring plan as listed in Tables 3 and 4. 
APPENDIX A 


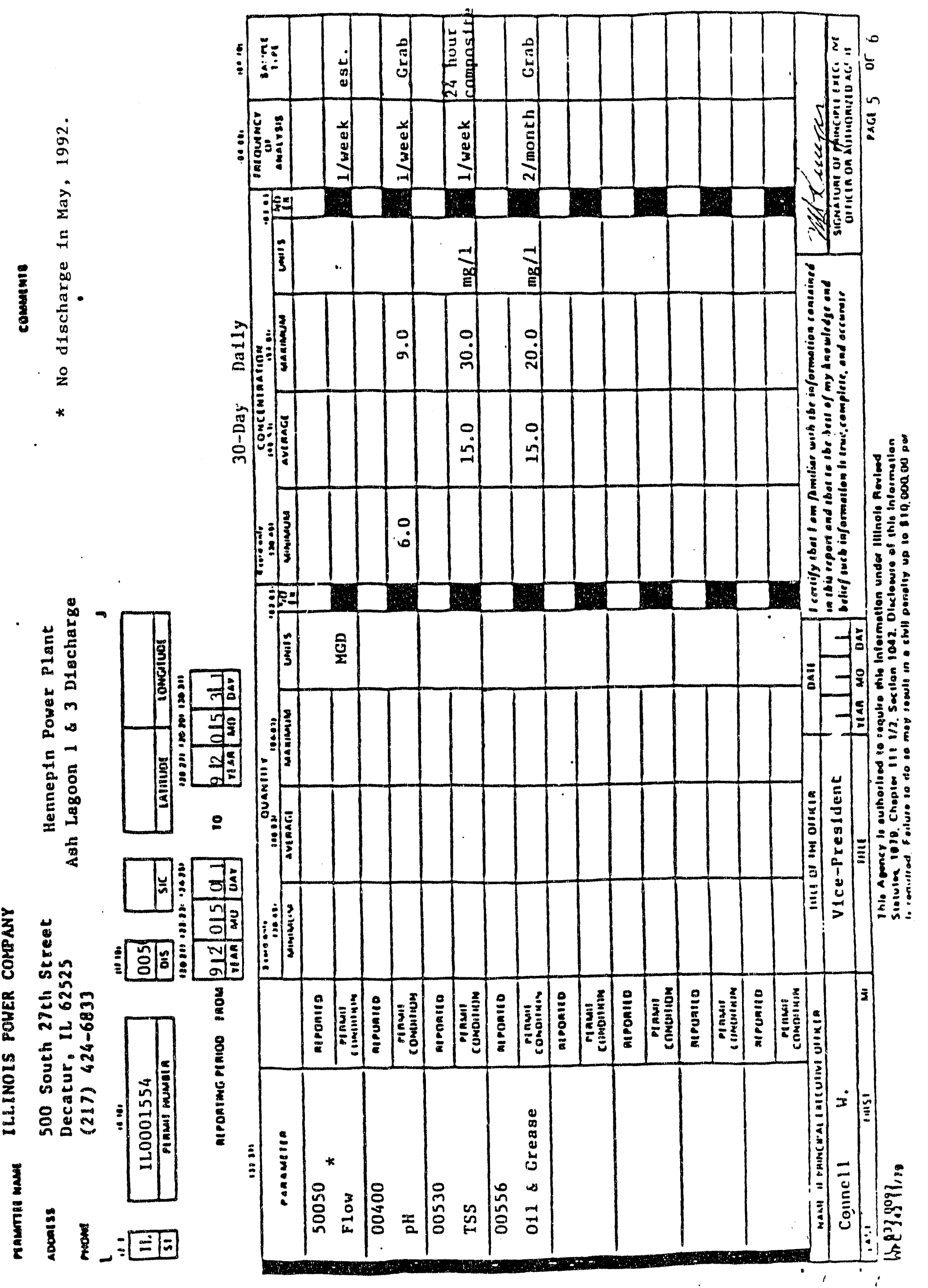




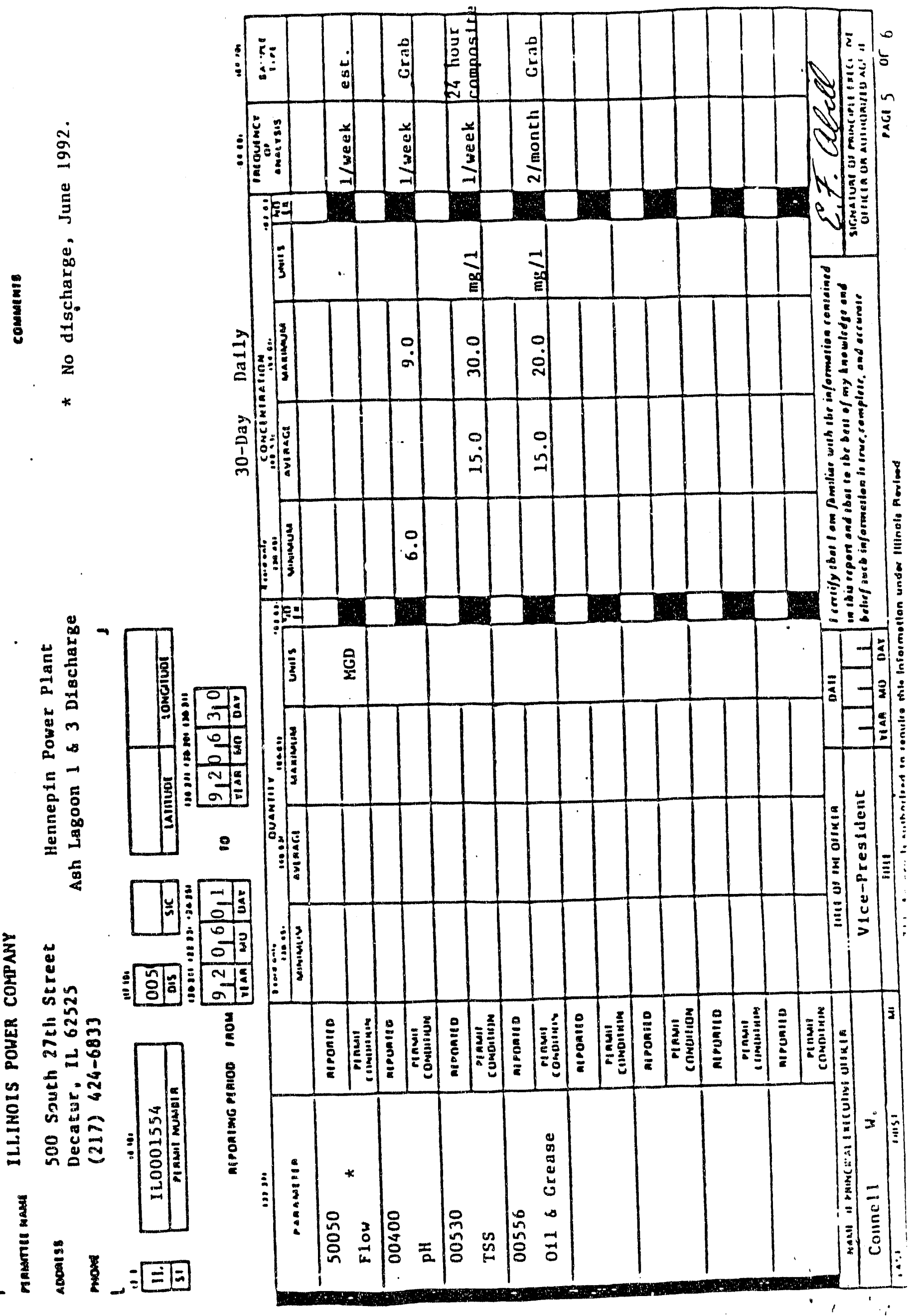




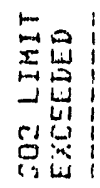

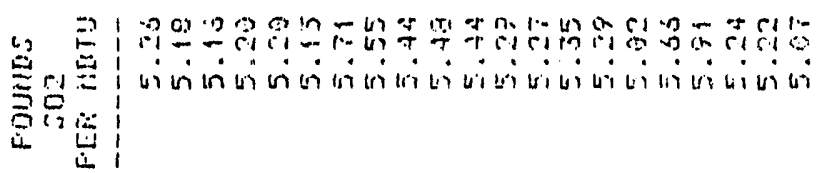

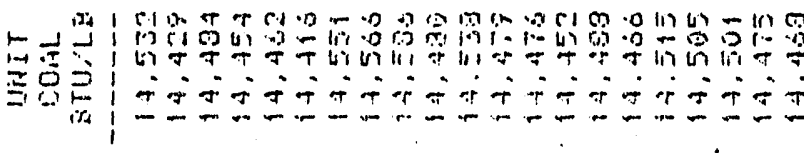

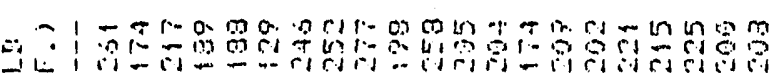

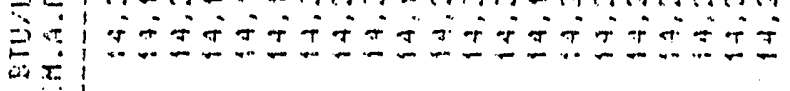

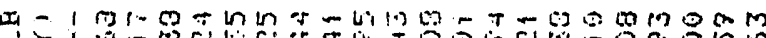

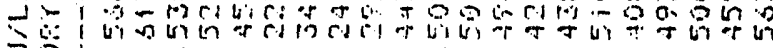

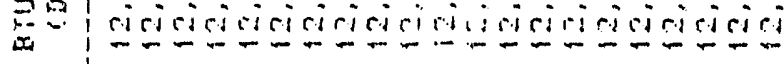

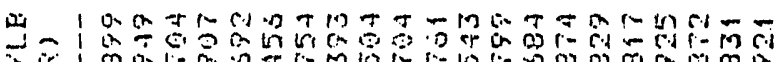

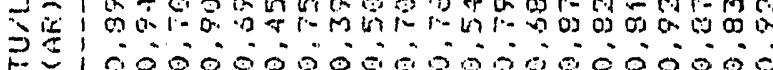
1

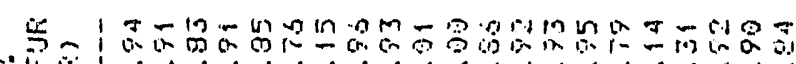

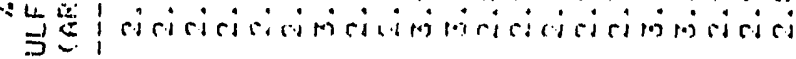

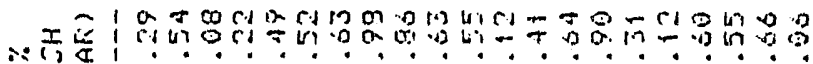

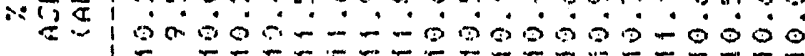

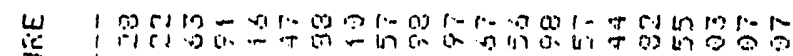
$\therefore F \hat{G}$ 量
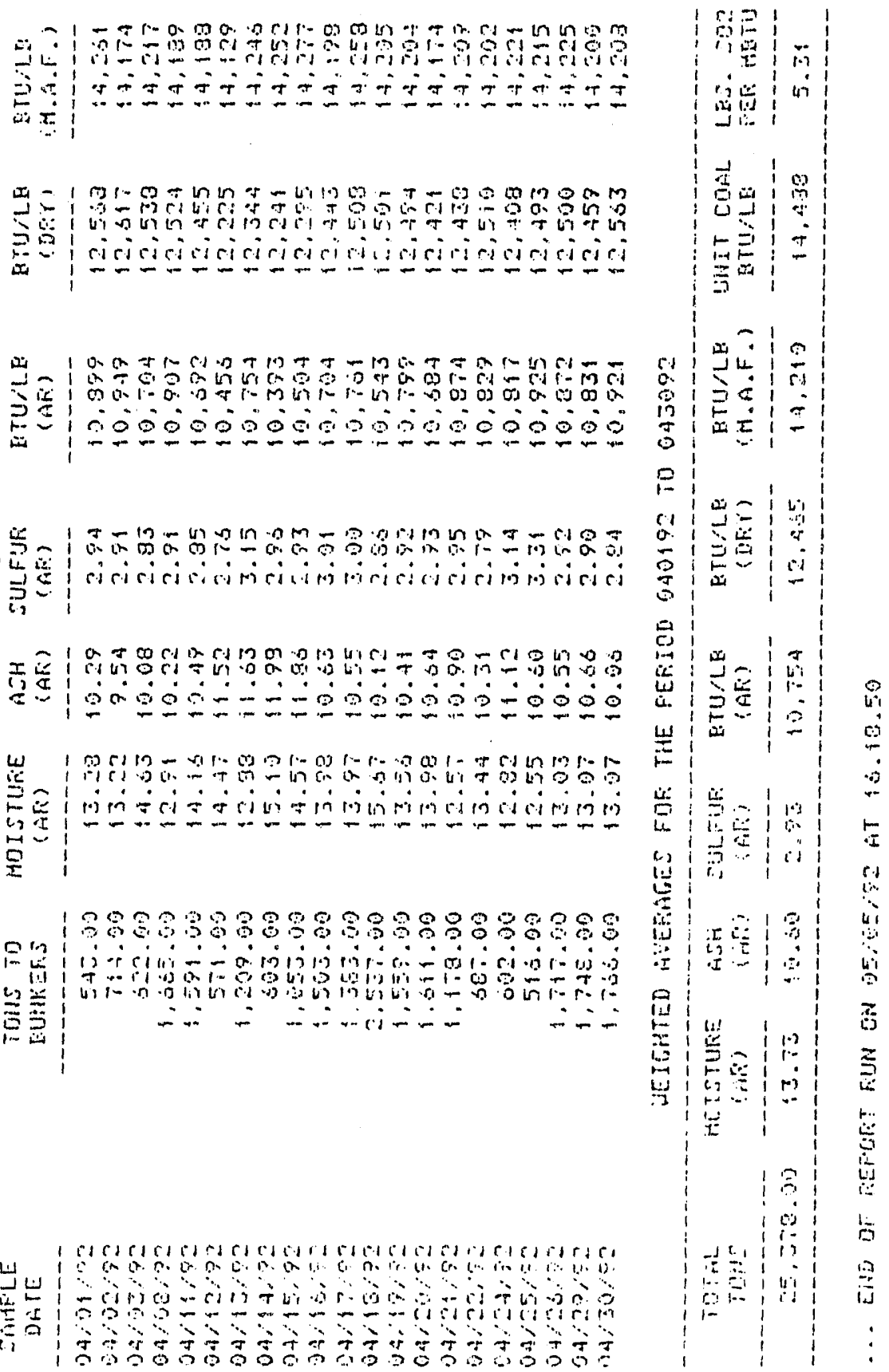

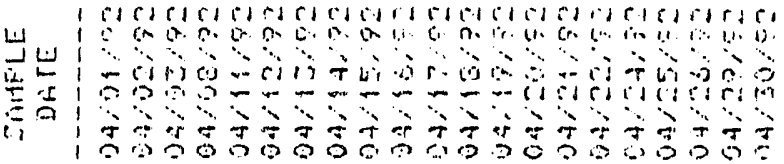




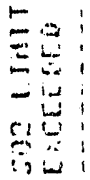

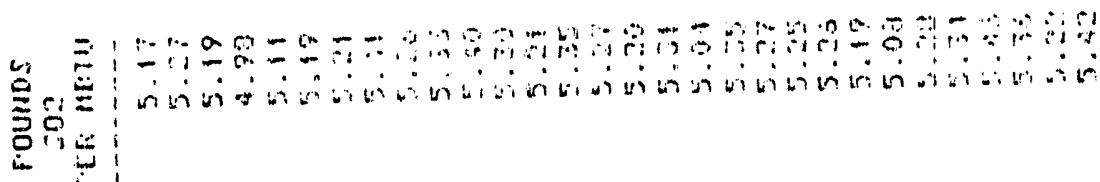

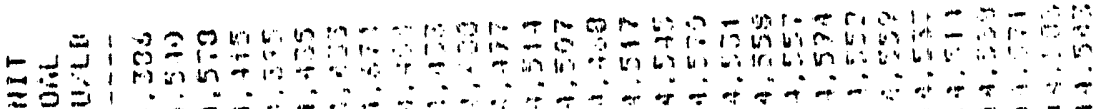

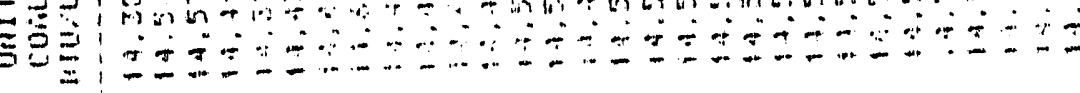

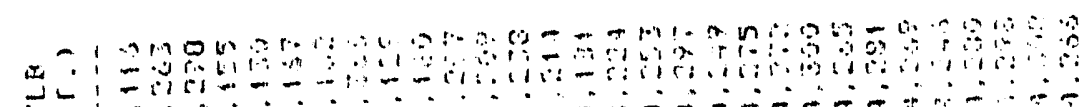

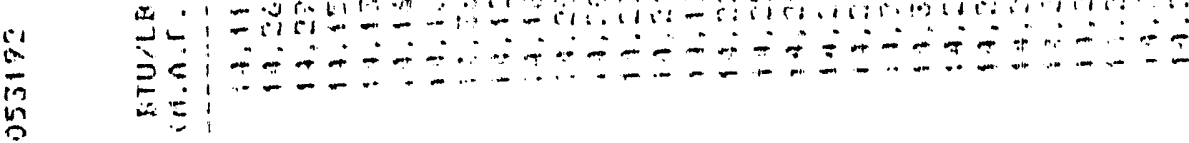

0

等

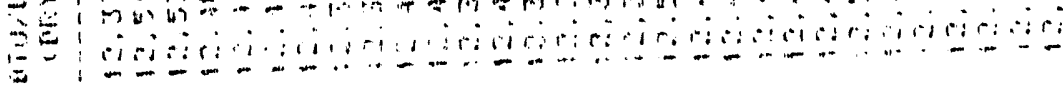

-

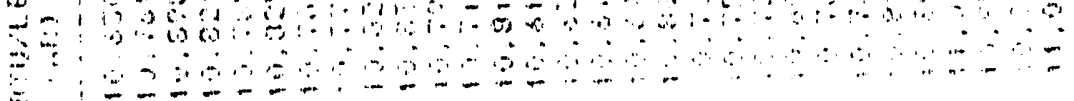

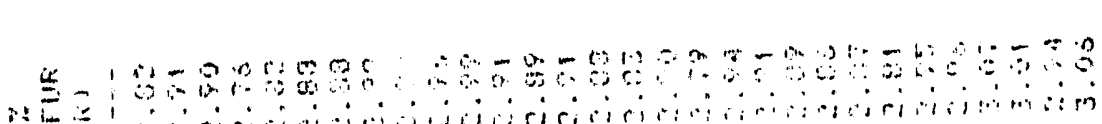

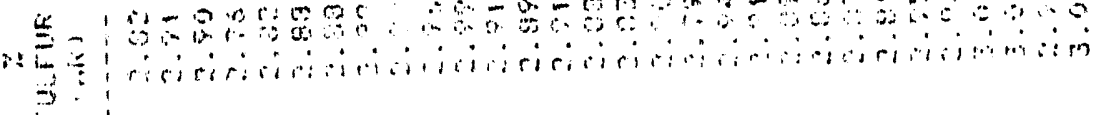

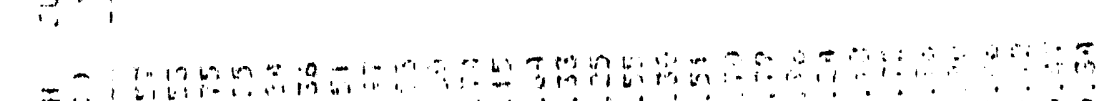
a $=\begin{aligned} & 0 \\ & 0\end{aligned}$

w 1 a

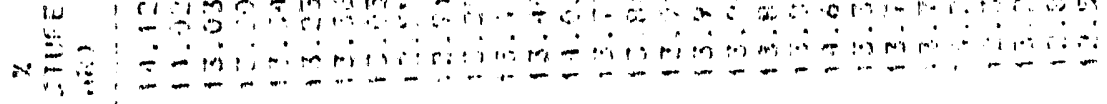
$\stackrel{c}{\underline{E}}$ e $0=-a_{0}$

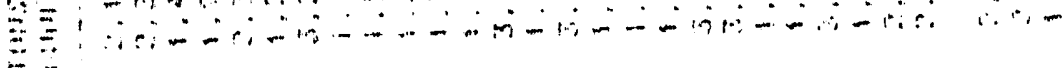

年 


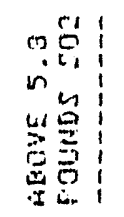

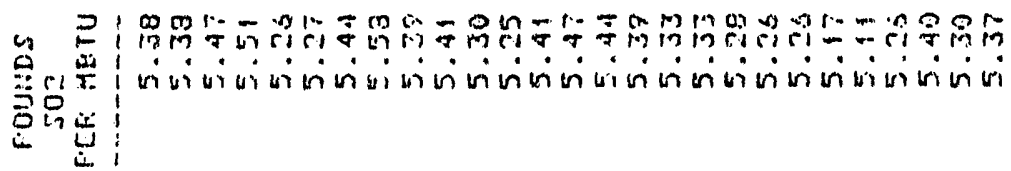

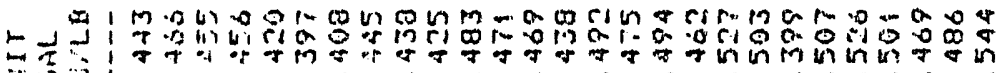

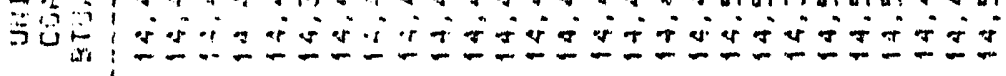

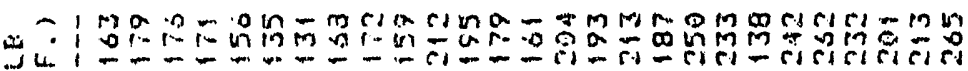

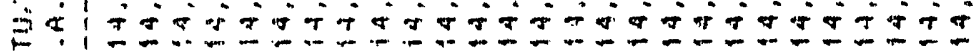

至

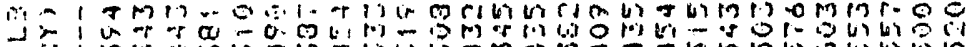

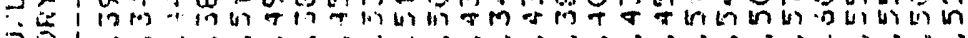

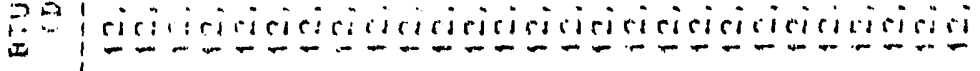

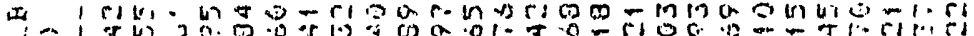

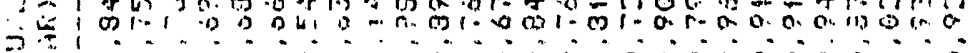

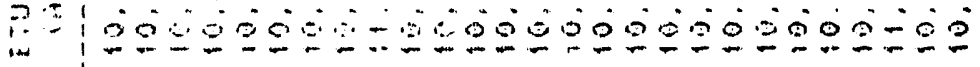

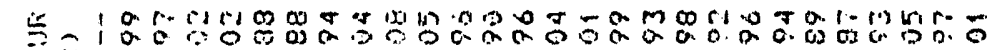

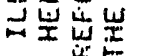

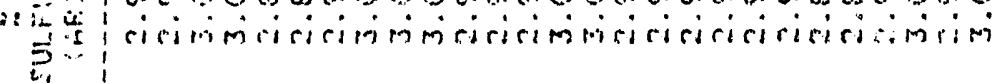

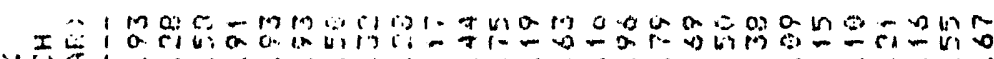

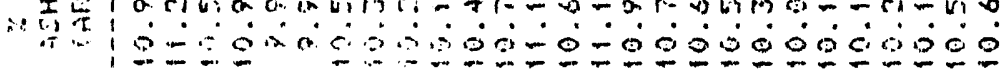

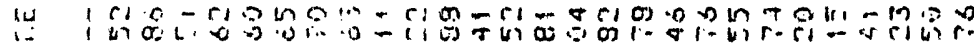

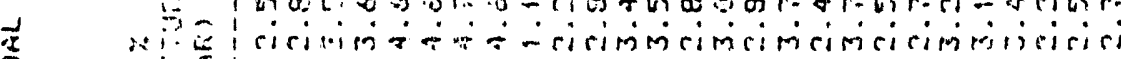
齐

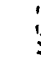

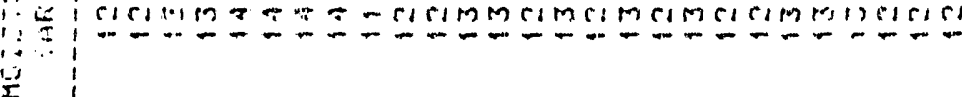

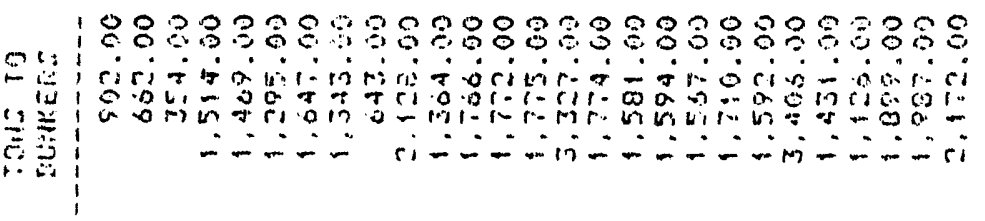

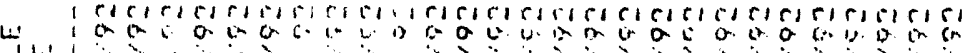

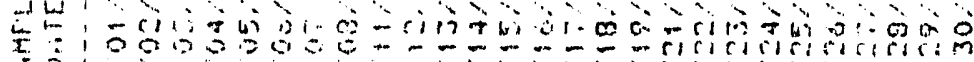
औ

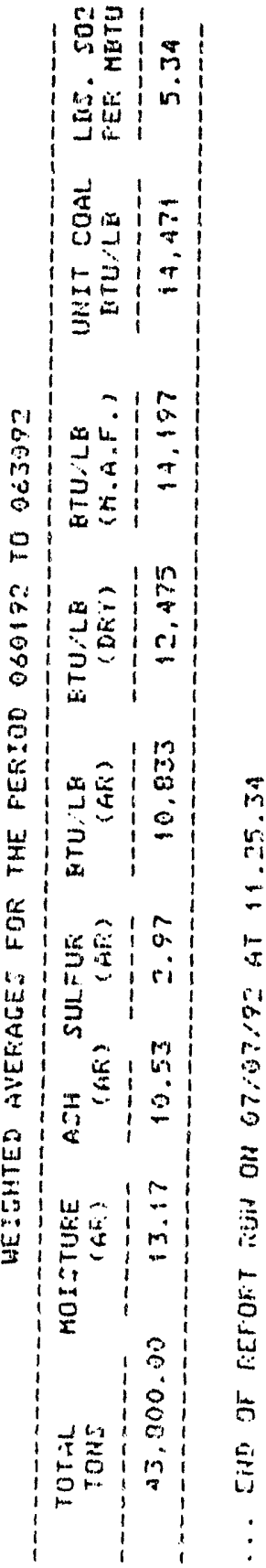


APPENDIX B 


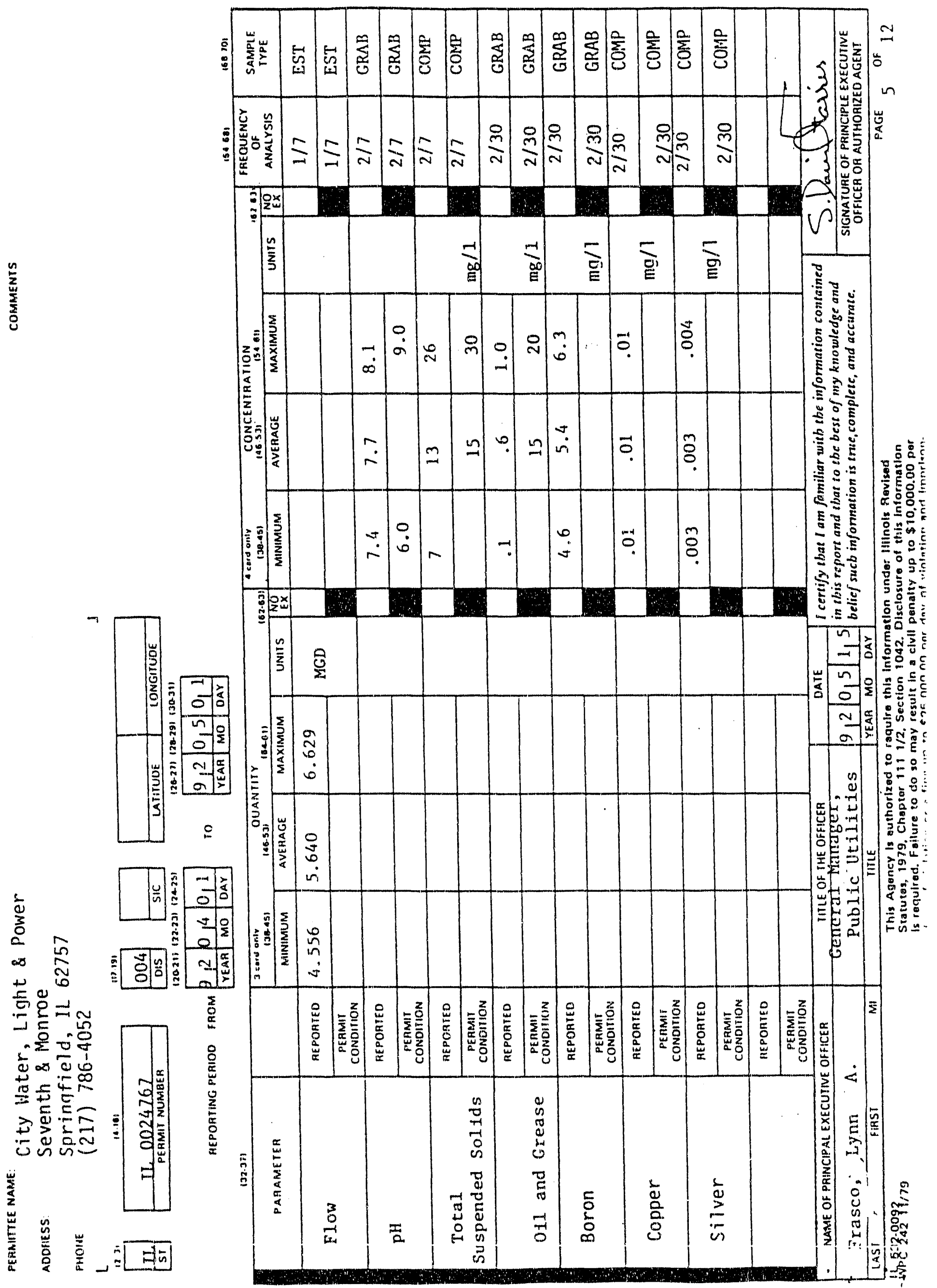




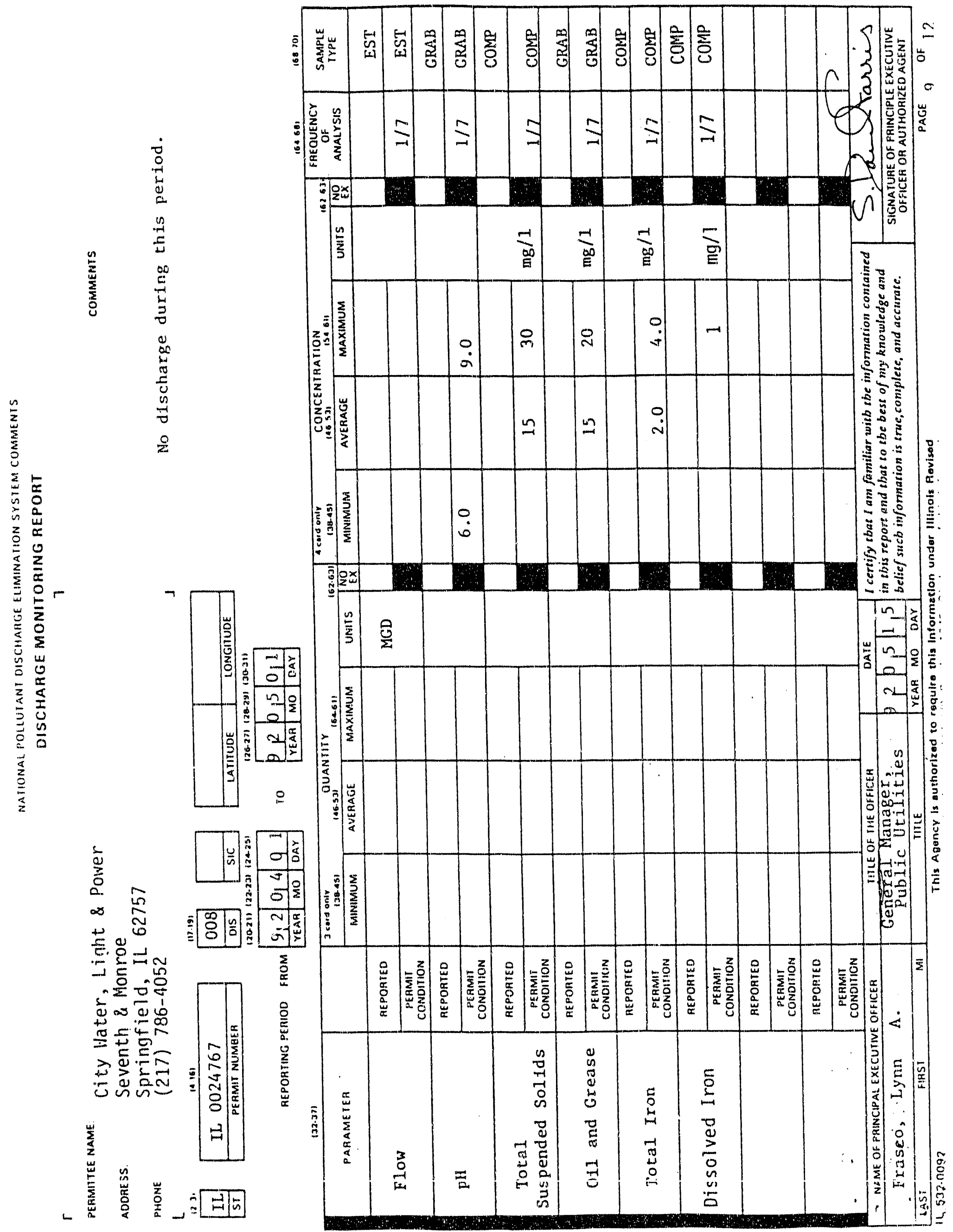




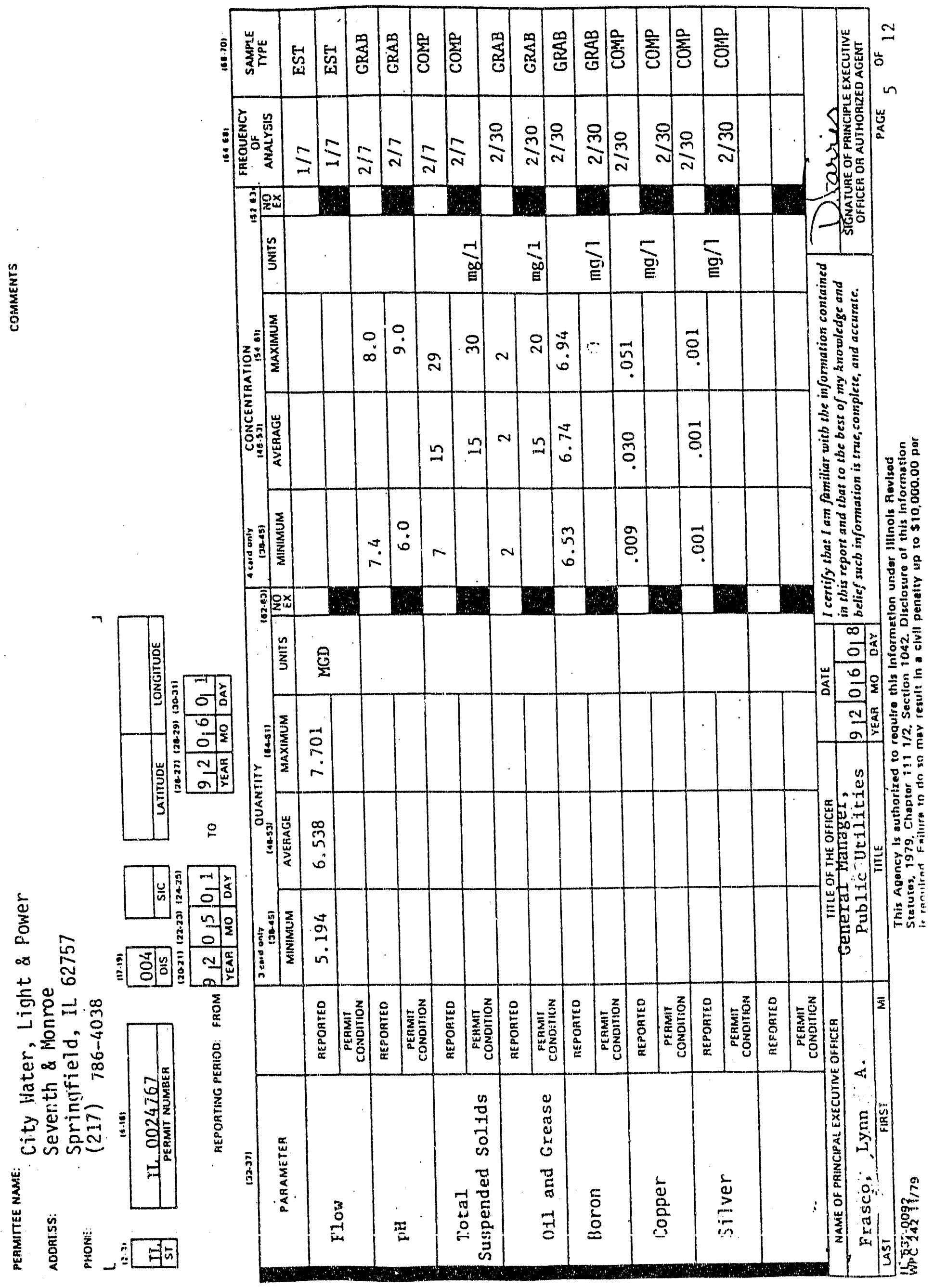




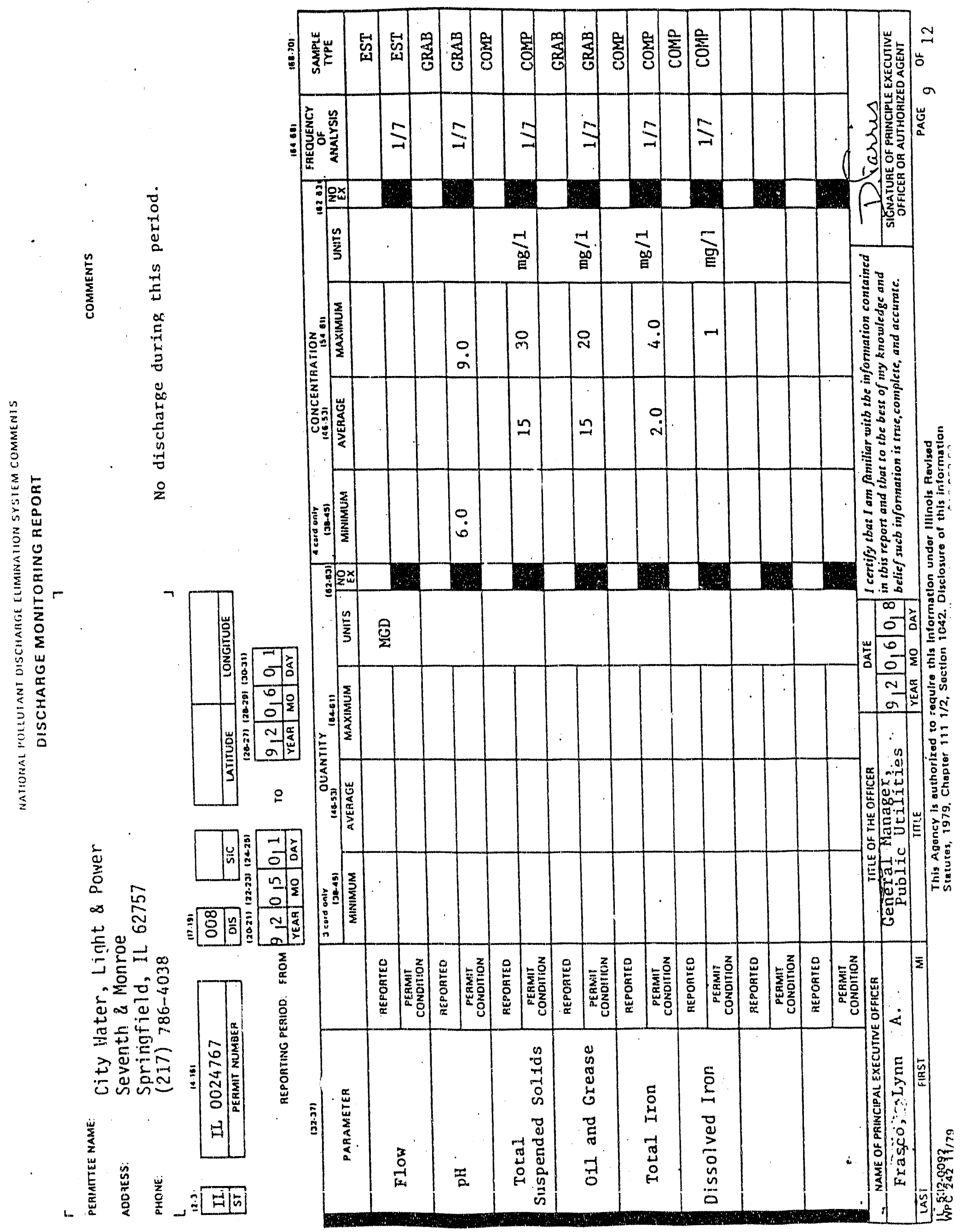




\section{CITY WAIEI, TIGKI \& POWIR \\ 3100 stevenson dxive \\ UNIY OPACITY CEM \\ DOWNTIML IREPORT}

Unit: Lakeside

Month/Year: April 1992

Day Start-End Component Cause $\quad \cdot \quad$ Corrective Action Taken
4/1/92 12:00A Clock Board Loose Wire
4/30/92 $\quad 23: 54$ Transmissometer

IEPA contacted \& notified on inoperativeness of Lakeside Opacity Monitor. 


\section{CITY WAIIR, IIGIT \& POWIER \\ 3100 steveran DIive}

\section{IXCESS OPACITY RIPORT}

Unit: Lakeside

Month/Year: April 1992

BOILER HOURS

Opacity Limit: $30 \%$

Unit $\$ 7-720$

Unit $\$ 8-720$ 


\section{CIMY WATER, IIGXIY \& POWER \\ 3100 stevensom Dxive \\ UNII OPACITY CDM \\ DOWNTIME FEPORY}

Jnit: $\quad 31$

Month/Year: April 1992

ay Start-End Component Cause Corrective Action Taken

11/92 12:00-08:50 Chart Recorder Paper Jam 


\section{CIIY WATER, LIGIT" \& POWFR \\ 3100 stevenaon Dxive}

\section{FRCESS OPACITY REM URT}

Unit: $\quad 31$

Month/Year: April 1992

Opacity Limit: $30 \%$

\begin{tabular}{|c|c|c|c|c|}
\hline Day & start & Opacity $\%$ & Cause & Corrective Action Taken \\
\hline $4-6$ & $\begin{array}{l}08: 54 \\
09: 00\end{array}$ & $\begin{array}{l}42.0 \\
42.0\end{array}$ & $\begin{array}{l}\text { Unknown } \\
\text { " }\end{array}$ & \\
\hline $4-11$ & $\begin{array}{l}02: 24 \\
02: 30 \\
02: 36 \\
03: 12\end{array}$ & $\begin{array}{l}68.0 \\
44.0 \\
31.0 \\
41.0\end{array}$ & $\begin{array}{l}\text { Maintenance Repair } \\
\text { on boiler forcing } \\
\text { precip. off. }\end{array}$ & \\
\hline $4-12$ & $\begin{array}{r}12: 00 \\
:: 06 \\
: 12 \\
: 18 \\
: 24 \\
: 30 \\
: 36 \\
: 42 \\
: 48 \\
: 54 \\
13: 00 \\
: 06 \\
: 12 \\
: 18 \\
: 24 \\
: 30 \\
: 36 \\
: 42 \\
: 48 \\
: 54 \\
14: 00 \\
: 06 \\
: 12 \\
: 18 \\
: 24 \\
: 30 \\
: 36 \\
: 42 \\
: 48 \\
: 54 \\
15: 00 \\
: 06 \\
: 12 \\
: 18 \\
: 24\end{array}$ & $\begin{array}{l}63.0 \\
54.0 \\
45.0 \\
61.0 \\
65.0 \\
67.0 \\
68.0 \\
71.0 \\
73.0 \\
75.0 \\
76.0 \\
78.0 \\
75.0 \\
79.0 \\
78.0 \\
77.0 \\
76.0 \\
74.0 \\
72.0 \\
69.0 \\
66.0 \\
63.0 \\
60.0 \\
57.0 \\
53.0 \\
50.0 \\
47.0 \\
44.0 \\
42.0 \\
39.0 \\
36.0 \\
48.0 \\
67.0 \\
59.0 \\
44.0\end{array}$ & 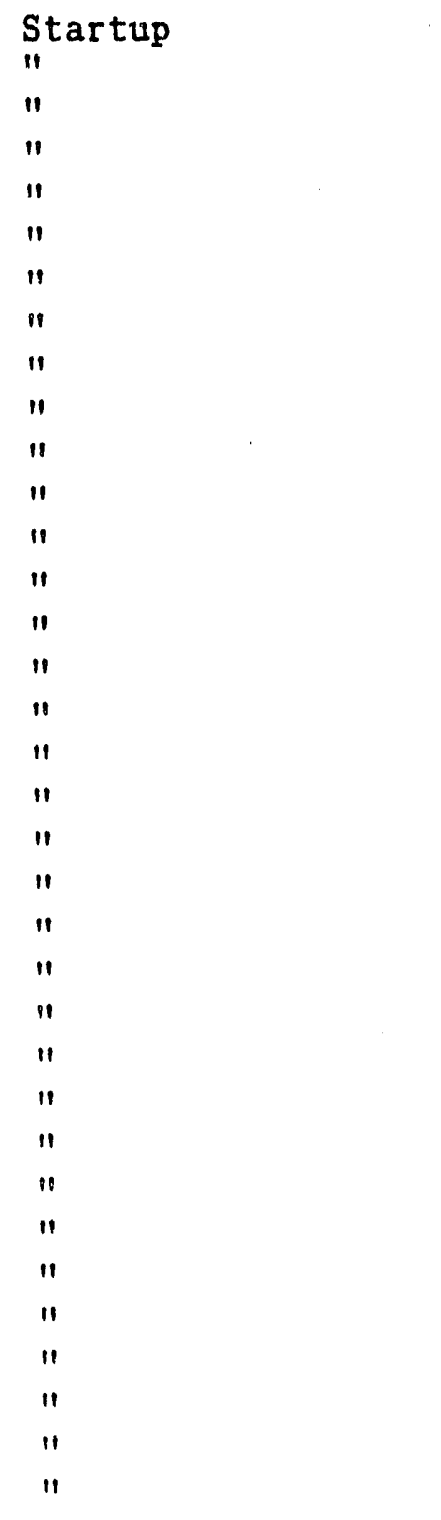 & \\
\hline
\end{tabular}


xcess Opacity Report

nit 31 - April 1992

age -2-

\begin{tabular}{|c|c|c|c|c|}
\hline Day & Start & Opacity $\%$ & Cause & Corrective Action Taken \\
\hline+-12 & $\begin{array}{r}15: 30 \\
: 36 \\
: 42 \\
: 48 \\
: 54 \\
16: 00 \\
: 06 \\
: 42 \\
: 48 \\
18: 42 \\
: 48 \\
: 54 \\
19: 00 \\
: 06 \\
: 12 \\
: 18 \\
:: 24 \\
: 30 \\
: 36 \\
; 44 \\
: 48 \\
: 54 \\
20: 00 \\
: 06 \\
: 112 \\
: 148 \\
: 24 \\
: 30 \\
: 36\end{array}$ & $\begin{array}{l}46.0 \\
43.0 \\
40.0 \\
50.0 \\
41.0 \\
43.0 \\
32.0 \\
43.0 \\
35.0 \\
47.0 \\
43.0 \\
32.0 \\
46.0 \\
71.0 \\
60.0 \\
66.0 \\
64.0 \\
64.0 \\
65.0 \\
68.0 \\
77.0 \\
74.0 \\
71.0 \\
67.0 \\
67.0 \\
67.0 \\
67.0 \\
67.0 \\
57.0\end{array}$ & 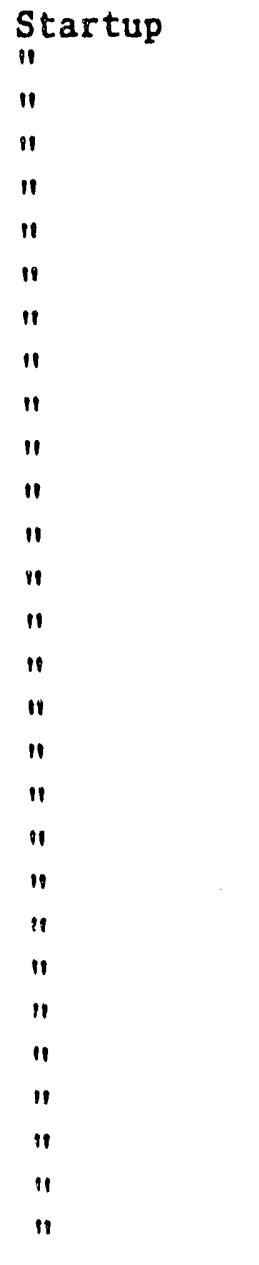 & ' \\
\hline $4-24$ & $20: 36$ & 37.0 & Shutdown & \\
\hline $4-26$ & $\begin{array}{r}01: 00 \\
: 06 \\
: 12 \\
: 18 \\
: 24 \\
: 30 \\
: 36 \\
: 42 \\
: 48 \\
: 54\end{array}$ & $\begin{array}{l}45.0 \\
79.0 \\
85.0 \\
53.0 \\
37.0 \\
35.0 \\
34.0 \\
33.0 \\
32.0 \\
31.0\end{array}$ & 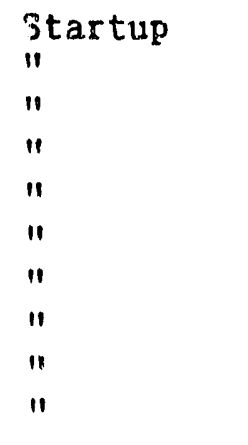 & . \\
\hline
\end{tabular}


xcess Opacity Report

nit 31 - April 1992

age $-3-$

Day Start Opacity $\%$ Cause Corrective Action Taken

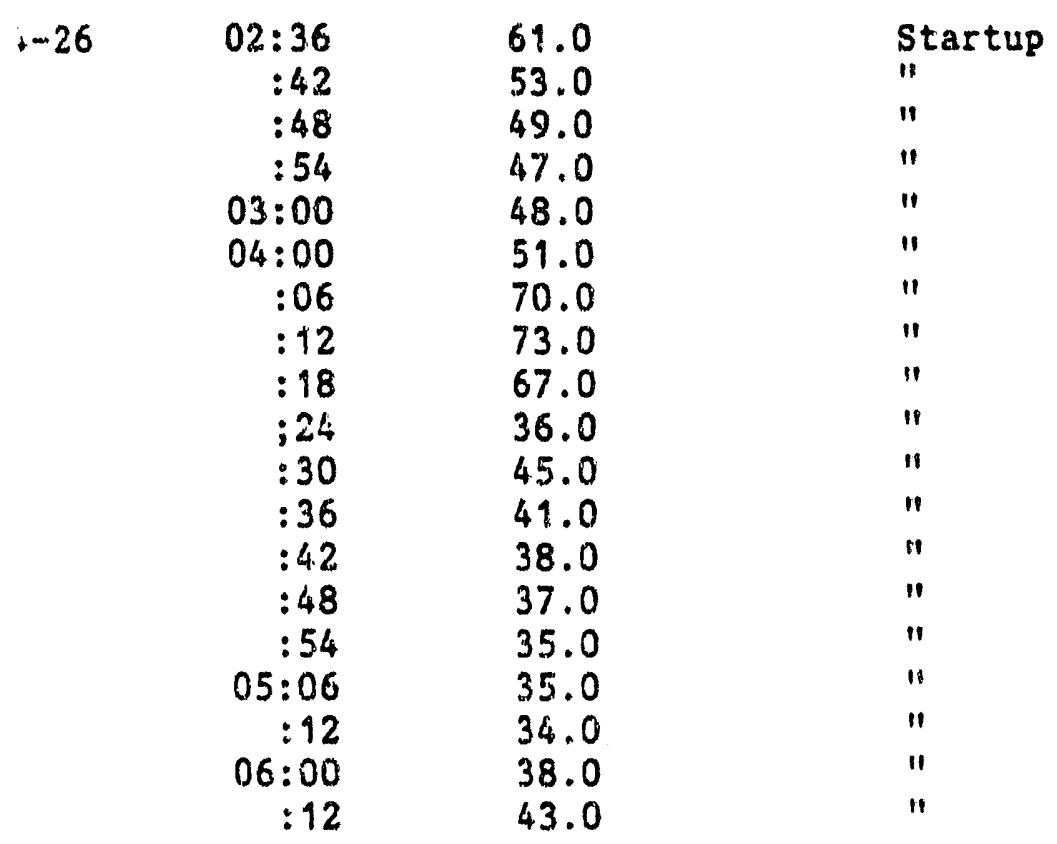




\section{CITY WATIE, IIGEI \& POWDR}

3100 steveraon Dxive

UnII OPACIYY CWM

DORNIIIME TEEPORT

Unit: $\quad 32$

Month/Year: April 1992

Day Start-End Component Cause $\quad \cdot \quad$ Corrective Action Taken

NON E 


\section{CIPY WAIIER, IIGIYT a POWER \\ 3100 stereanon Drive \\ PRCIFS ORACITY IREPORT}

Unit: $\quad 32$

Month/Year: April 1992

Opacity Limit: $30 \%$

Day Start opacity $\%$ Cause $\quad$ Corrective Action Taken

$\begin{array}{rrrl}4-6 & 08: 30 & 72.0 & \text { Precipitator Malfunction } \\ & : 36 & 72.0 & " 1 \\ : 42 & 72.0 & " 1 \\ & : 48 & 72.0 & \\ & : 54 & 72.0 & \\ & & & \\ 4-27 & 16: 42 & 55.0 & \text { Unknown } \\ & 24: 36 & 40.0 & \end{array}$




\section{CIWY WATIR, TIGET \& POWTAR \\ 3100 steveremon Drive \\ UNII OPACITX CDM \\ DONATIMA REPORT}

Unit: $\quad 33$

Month/Year: April 1992

Day Start-End Component

UNIT OUTAGE 


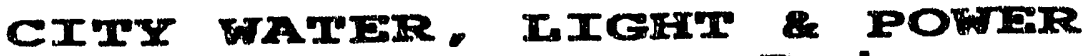 \\ 3100 stevenson Drive \\ EXCESS OPACIYY IREPORT}

Unit: $\quad 33$

Month/Year: April 1992

Opacity Iimit: $30 \%$

Jay Start Opacity $\%$

Cause

Corrective Action Taken

UNIT ODTAGE 


\section{CIIY WATER, LIGIY \& FOWIBR}

3100 stevermon Drive

\section{UNIT OPACITY CFM}

DOWNTIME REPORT

Unit: Lakeside

Month/Year: May 1992

\begin{tabular}{lccc}
$: y$ & Start-End & Component & Cause \\
\hline 1 & $12: 00 \mathrm{~A}-$ & Transmissometer & Cal Fail \\
4 & $07: 30$ & &
\end{tabular}
29
$09: 24-11: 00$
Opacity Recorder
Stuck in $\mathrm{Cal}$

\author{
Measured stack \& recali- \\ brated instrument
}




\section{CIMY WAMWR, IIGIIT \& POWTER \\ 3100 stevenson Dxive \\ FXCFSS OPACIMY REFORT}

Init: Lakeside

lonth/Year: April 1992

BOTLRR HOURS

Jpacity Limit: $30 \%$

Onit $\| 7-619$

Unit \#8-608

\begin{tabular}{|c|c|c|c|c|}
\hline ay & start & Opacity $\%$ & Cause & Corrective Action Taken \\
\hline-13 & $\begin{array}{r}18: 54 \\
19: 00 \\
: 06 \\
: 12\end{array}$ & $\begin{array}{l}41.0 \\
74.0 \\
74.0 \\
66.0\end{array}$ & $\begin{array}{l}\text { Unit } 7 \text { Trip } \\
" 1 " \\
" 1\end{array}$ & . \\
\hline-17 & $\begin{aligned} 12 & : 18 \\
: & 24 \\
: & 30\end{aligned}$ & $\begin{array}{l}64.0 \\
76.0 \\
32.0\end{array}$ & $\begin{array}{l}\text { Precip. field tripped } \\
" 1\end{array}$ & Reset field \\
\hline-18 & $11: 18$ & 31.0 & Unknown & \\
\hline-21 & $\begin{aligned} 21: & : 36 \\
: & 42 \\
& : 48 \\
22 & : 00 \\
& : 06 \\
: & : 12 \\
: & 24 \\
: & 48 \\
: & 54 \\
23: & 00 \\
: & 06 \\
: & : 12 \\
: & 18\end{aligned}$ & $\begin{array}{l}41.0 \\
46.0 \\
42.0 \\
57.0 \\
76.0 \\
61.0 \\
36.0 \\
47.0 \\
37.0 \\
31.0 \\
42.0 \\
55.0 \\
40.0\end{array}$ & $\begin{array}{l}\text { Equip. Malf./Air } \\
\text { Damper } \\
\text { " } \\
\text { " } \\
\text { " } \\
\text { " } \\
\text { " } \\
\text { " } \\
\text { " } \\
\text { " }\end{array}$ & $\begin{array}{l}\text { Placed in manual } \\
\text { control until repairs } \\
\text { were completed } \\
\text { " } \\
\text { " } \\
" \\
" \\
" 1 \\
" \\
" 1 \\
"\end{array}$ \\
\hline-29 & $\begin{array}{r}18: 54 \\
19: 30 \\
: 36 \\
: 42 \\
: 48 \\
: 54 \\
20: 00 \\
: 06 \\
: 12 \\
: 18 \\
: 24 \\
: 30 \\
: 36 \\
: 42 \\
: 48 \\
: 54\end{array}$ & $\begin{array}{l}63.0 \\
74.0 \\
80.0 \\
80.0 \\
78.0 \\
71.0 \\
75.0 \\
75.0 \\
76.0 \\
78.0 \\
79.0 \\
71.0 \\
63.0 \\
61.0 \\
55.0 \\
52.0\end{array}$ & $\begin{array}{l}\text { Startup } 7 \& 8 \\
" 1 \\
" 1 \\
" 1 \\
" 1 \\
" 1 " \\
" \\
" \\
" \\
" \\
" \\
" \\
"\end{array}$ & \\
\hline
\end{tabular}


cess Opacity Report

keside - May 1991

ge -2-

\begin{tabular}{|c|c|c|c|c|}
\hline Day & Start & Opacity $\%$ & Cause & Corrective Action Taken \\
\hline 29 & $\begin{array}{r}21: 00 \\
: 06 \\
: 12 \\
: 18 \\
: 24 \\
: 30 \\
: 36 \\
: 42 \\
: 48 \\
22: 24 \\
: 30 \\
: 36 \\
: 42 \\
: 48 \\
: 54 \\
23: 00 \\
: 24 \\
: 36 \\
: 42 \\
: 54\end{array}$ & $\begin{array}{l}52.0 \\
34.0 \\
31.0 \\
45.0 \\
47.0 \\
42.0 \\
37.0 \\
41.0 \\
34.0 \\
40.0 \\
57.0 \\
57.0 \\
48.0 \\
54.0 \\
42.0 \\
35.0 \\
39.0 \\
32.0 \\
50.0 \\
34.0\end{array}$ & 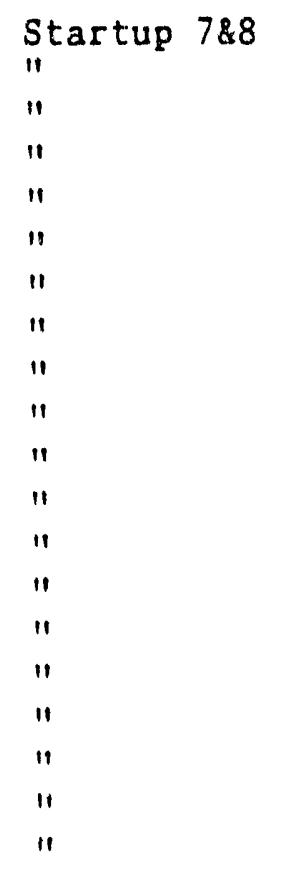 & \\
\hline-30 & $\begin{aligned} 00 & : 36 \\
& : 54 \\
01: & 12 \\
: & 30 \\
: & 54\end{aligned}$ & $\begin{array}{l}37.0 \\
43.0 \\
32.0 \\
45.0 \\
42.0\end{array}$ & $\begin{array}{l}\text { Startup } 7 \& 8 \\
" 1 \\
" 1 \\
\text { " }\end{array}$ & \\
\hline
\end{tabular}




\section{CIMY MATEIR. IIGXIT \& POWPR}

3100 sterenson Drive

\section{UNIX OPACIMY CEM}

DOWNTIMTE IREPORT

Jnit: $\quad 31$

Ionth/Year: May 1992

ay Start-End Component

Cause

Corrective Action Taken

N $O N E$ 


\section{CITY WATER, IIGFT \& POWIFR}

3100 stevenaon Dxive

\section{FXCESS OPACITY IRPORT"}

Unit: $\quad 31$

Month/Year: May 1992

Opacity Limit: $30 \%$

\begin{tabular}{rrrl} 
ay & Start & Opacity $\%$ & \multicolumn{1}{c}{ Cause } \\
\hline-11 & $00: 06$ & 49.0 & Switched Precip.Fields \\
& $: 12$ & 58.0 & "
\end{tabular}

Corrective Action Taken 


\section{CITY WATER, IIGFI \& POWER}

3100 steveraon Dxive

UNII OPACIMY CEM

DOWNTIMEF REPORT

nit: $\quad 32$

onth/Year: May 1992

y Start-End 


\section{CITY WATER, IIGITI \& POWLPR}

\section{0 stevenson Dxive}

FXCFSS OPACIMY RPPORI

Jnit: $\quad 32$

1onth/Year: May 1992

Opacity Limit: $30 \%$

\begin{tabular}{|c|c|c|c|c|}
\hline ay & Start & Opacity \% & Cause & Corrective Action Taken \\
\hline-12 & $\begin{aligned} 00: & : 06 \\
& : 12\end{aligned}$ & $\begin{array}{l}50.0 \\
89.0\end{array}$ & Switched Precip. Fields & \\
\hline-21 & $\begin{array}{r}02: 48 \\
03: 00 \\
: 06 \\
06: 54 \\
07: 00 \\
: 06 \\
: 12 \\
: 18 \\
: 24 \\
: 30 \\
: 36 \\
: 42 \\
: 48 \\
: 54 \\
08: 00 \\
: 06 \\
: 42 \\
09: 12 \\
: 18 \\
10: 36\end{array}$ & $\begin{array}{l}62.0 \\
33.0 \\
36.0 \\
60.0 \\
64.0 \\
54.0 \\
47.0 \\
52.0 \\
63.0 \\
65.0 \\
59.0 \\
55.0 \\
53.0 \\
53.0 \\
53.0 \\
35.0 \\
59.0 \\
70.0 \\
89.0 \\
35.0\end{array}$ & $\begin{array}{l}\text { Startup } \\
\text { " } \\
" \\
" \\
" \\
" \\
" \\
" \\
" \\
" \\
" \\
" \\
" \\
" \\
" \\
" \\
" \\
" \\
"\end{array}$ & . \\
\hline $5-25$ & $\begin{aligned} & 08: 54 \\
& 09: 00 \\
&: 06 \\
&: 12 \\
&: 18 \\
&: 24 \\
&: 30 \\
&: 36 \\
& 11: 12\end{aligned}$ & $\begin{array}{l}71.0 \\
40.0 \\
49.0 \\
49.0 \\
46.0 \\
43.0 \\
40.0 \\
36.0 \\
31.0\end{array}$ & $\begin{array}{l}\text { Startup } \\
\text { " } \\
\text { " } \\
" \\
" \\
" \\
" \\
"\end{array}$ & \\
\hline $5-29$ & $\begin{aligned} & 10: 00 \\
&: 06 \\
&: 12 \\
&: 18 \\
&: 24 \\
&: 30 \\
&: 36\end{aligned}$ & $\begin{array}{l}79.0 \\
73.0 \\
58.0 \\
53.0 \\
49.0 \\
41.0 \\
49.0\end{array}$ & $\begin{array}{l}\text { Startup } \\
\text { " } \\
" 1 \\
" \\
" \\
\text { " }\end{array}$ & \\
\hline
\end{tabular}


:ess. Opacity Report

it 32 - May 1992

ze -2-

Jay Start Opacity $\%$

Cause

Corrective Action Taken

29

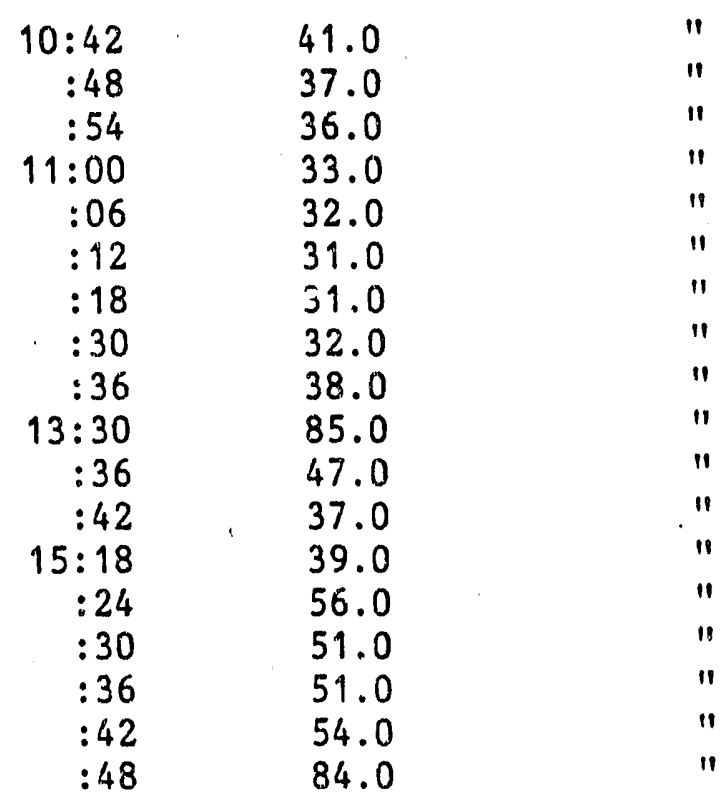




\section{CITY WATER, IIGEIS \& POWDR}

3100 stevenson Dxive

UNIT OPACITY CDAX

DOWNTIME REPORT

Unit: $\quad 33$

Month/Year: May 1992

iay Start-End Component

Cause

Corrective Action Taken

120 15:00-20:00 Opacity Recorder Paper Jam

$\begin{array}{llll}123 & \text { Malfunctioning chopper } & \text { Replaced motor \& } \\ \text { 125 } & \text { Transmissometer } & \text { motor } & \text { recalibrated }\end{array}$




\section{CIIY WMIBR, IICITY a POWIR \\ 3100 Btevenson Dxive}

DXCESS OPACITY REPORT

nit: 33

Inth/Year: May 1992

Pacity Limit: $30 \%$

Start Opacity \%

Cause

Corrective Action Taken

1

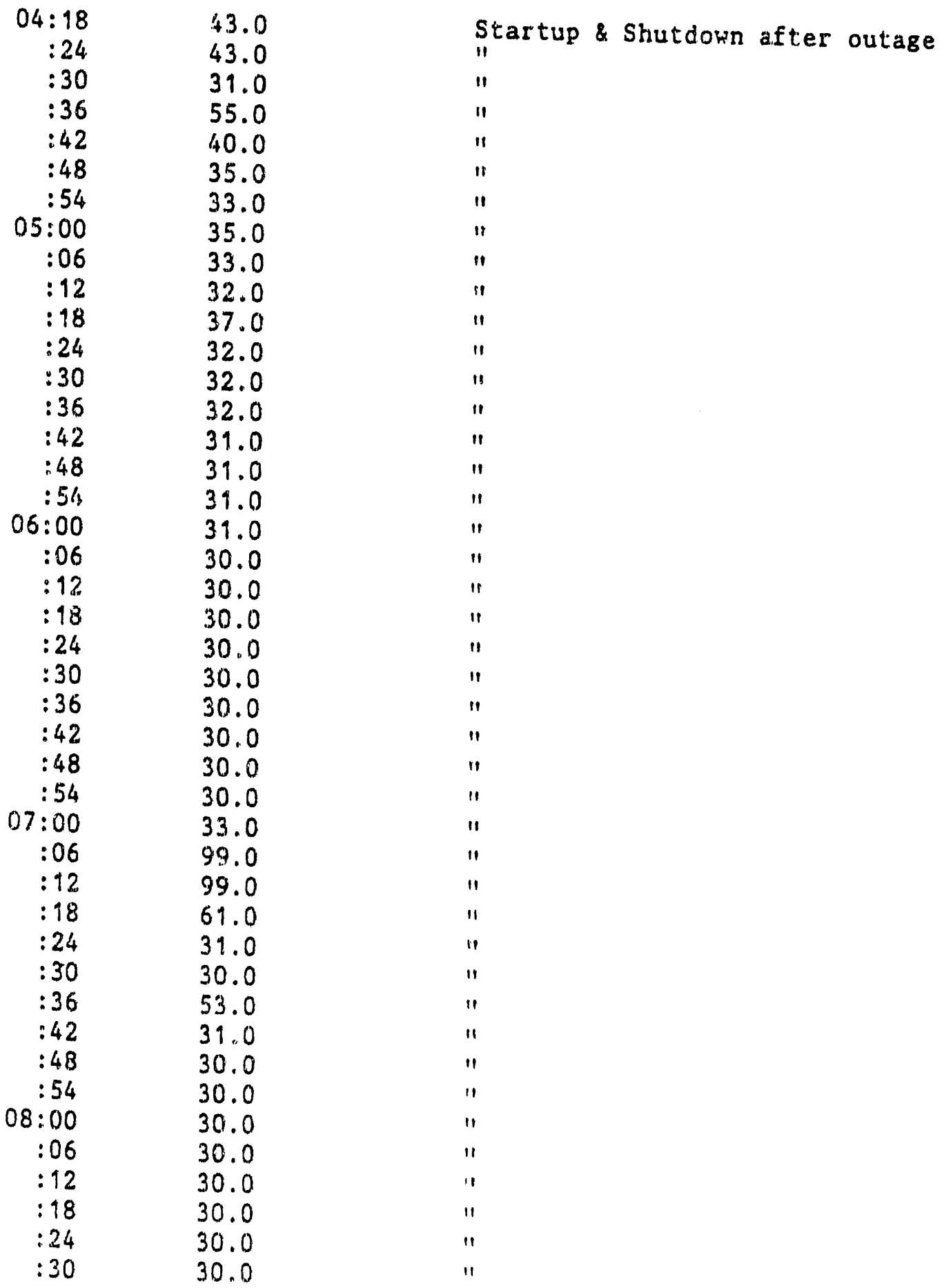


zess Opacity Report

it 33.- May 1992

se $-2-$

Jay Start Opacity $\%$ Cause Corrective Action Taken

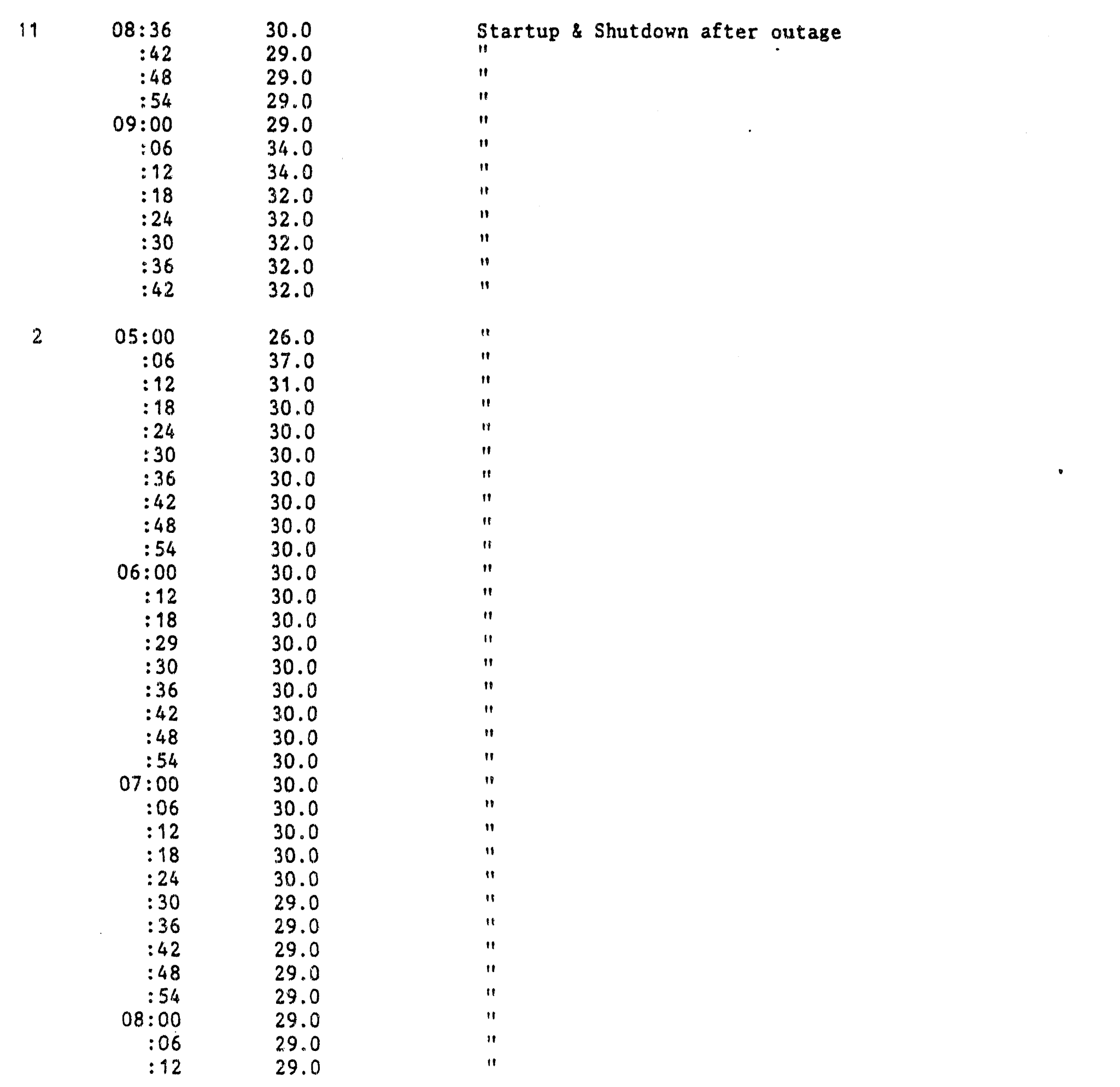


:cess Opactity Report

iit 33 - May 1992

ige -3-

Day Start Opacity $\%$ Cause Corrective Action Taken

\begin{tabular}{|c|c|c|}
\hline $\begin{aligned} & 08: 18 \\
&: 29 \\
&: 30 \\
&: 36 \\
&: 42 \\
&: 48 \\
&: 54 \\
& 09: 00 \\
&: 06 \\
&: 12 \\
&: 18 \\
&: 24 \\
&: 30\end{aligned}$ & $\begin{array}{l}29.0 \\
29.0 \\
29.0 \\
29.0 \\
29.0 \\
29.0 \\
29.0 \\
29.0 \\
29.0 \\
29.0 \\
29.0 \\
29.0 \\
29.0\end{array}$ & $\begin{array}{l}\text { Startup \& Shutdown after outage } \\
" 1 \\
" \\
" \\
" \\
" \\
" \\
" \\
" \\
" \\
"\end{array}$ \\
\hline
\end{tabular}

\begin{tabular}{|c|c|c|c|}
\hline-15 & $\begin{array}{r}12:: 18 \\
: 24 \\
: 30 \\
: 36 \\
;: 42 \\
:: 48 \\
14: 42 \\
: 48 \\
18: 24 \\
19: 48 \\
20: 00 \\
21: 36 \\
:: 42 \\
22: 18 \\
: 54\end{array}$ & $\begin{array}{l}47.0 \\
82.0 \\
73.0 \\
33.0 \\
27.0 \\
23.0 \\
23.0 \\
25.0 \\
21.0 \\
21.0 \\
24.0 \\
21.0 \\
22.0 \\
23.0 \\
21.0\end{array}$ & 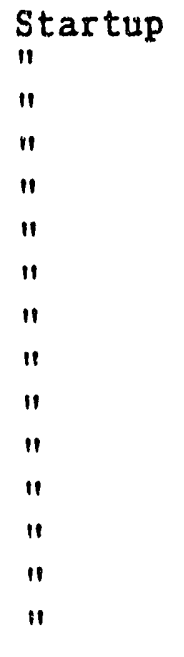 \\
\hline-16 & $\begin{aligned} & 00: 06 \\
&: 12 \\
&: 18 \\
& 01: 42 \\
&:: 48 \\
&: 54 \\
& 02: 00 \\
& 12: 18 \\
&:: 24 \\
&: 30 \\
&: 36 \\
&: 42 \\
&: 48 \\
&: 54\end{aligned}$ & $\begin{array}{l}22.0 \\
22.0 \\
22.0 \\
21.0 \\
21.0 \\
21.0 \\
21.0 \\
23.0 \\
23.0 \\
23.0 \\
23.0 \\
23.0 \\
23.0 \\
23.0\end{array}$ & $\begin{array}{l}\text { Startup } \\
" 1 \\
" 1 \\
" 1 \\
" 1 \\
" 1 \\
" 1 \\
" 1 \\
" 1 \\
" 1 \\
" 1\end{array}$ \\
\hline
\end{tabular}


zess Opacity Report

it 33 - May 1992

se $-4-$

Day start Opacity $\%$

16

$6 \quad 13: 00$

23.0

23.0

$: 12$

22.0

$14: 06$

23.0

\section{Startup}

i1

18

$$
\begin{array}{r}
21: 36 \\
: 42 \\
: 48 \\
: 54
\end{array}
$$

$22: 00$

$: 06$

$: 12$

$: 18$

$: 24$

$: 30$

$: 36$

$: 42$

$: 48$

$: 54$

$23: 00$

$: 06$

$: 12$

$: 18$

$: 24$

$: 30$

$: 36$

$: 42$

$: 48$

$: 54$

31.0

82.0

77.0

73.0

68.0

75.0

51.0

45.0

47.0

42.0

42.0

36.0

35.0

47.0

48.0

33.0

30.0

30.0

32.0

29.0

26.0

28.0

76.0

59.0

19

$00: 00$
$: 06$
$: 12$
$: 18$

$: 24$

$03: 30$

$: 36$

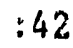

$: 48$

$: 54$

$04: 00$

$: 06$

$: 12$

:18

;24

$04: 48$

$: 54$

35.0

40.0

43.0

32.0

21.0

24.0

23.0

41.0

41.0

41.0

41.0

45.0

46.0

44.0

42.0

45.0

45.0 
cess Opacity Report

it 3,3. - May 1992

ge -5-

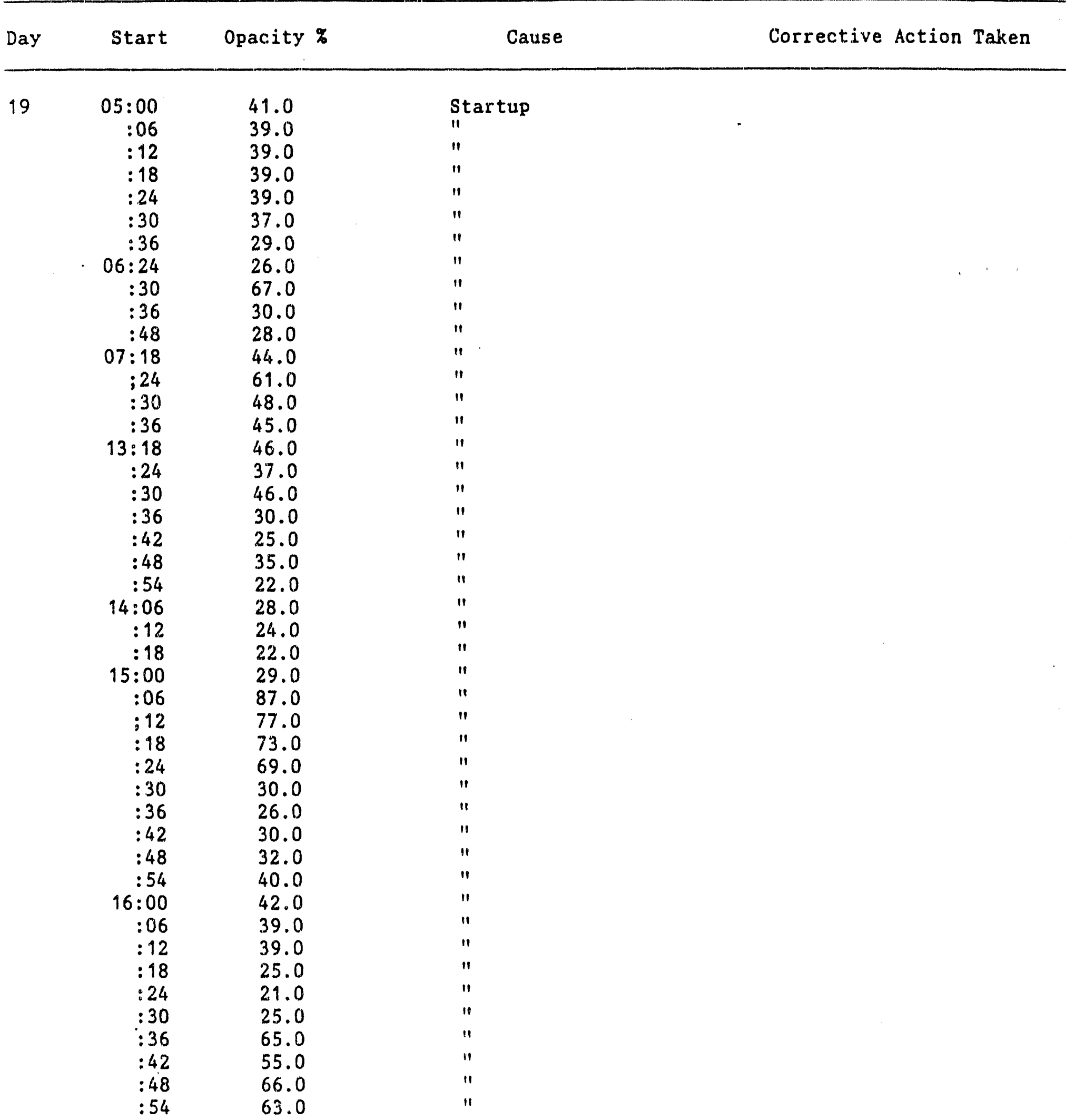


ess Opaci'ty Report

t 33 - May 1992

e $-6-$

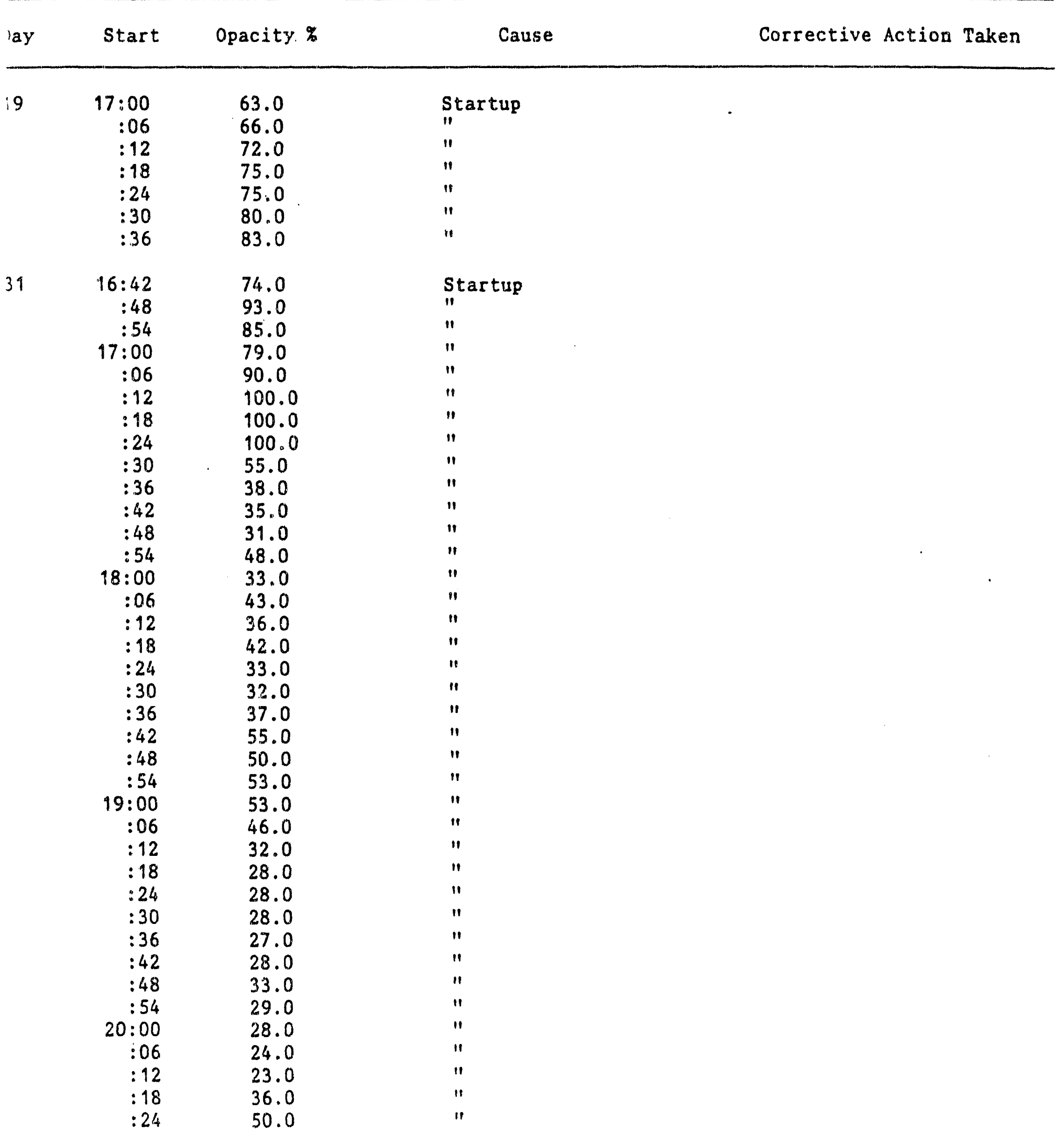


zess Opacity Report

it 33 - May 1992

ge -7-

\begin{tabular}{|c|c|c|c|}
\hline Day & Start & Opacity \% & $\mathrm{Ca}$ \\
\hline 31 & $\begin{array}{r}20: 30 \\
: 36 \\
: 42 \\
: 48 \\
: 54 \\
21: 00 \\
: 06 \\
: 30 \\
: 36 \\
: 42 \\
: 48 \\
: 54 \\
22: 00 \\
: 06 \\
: 12 \\
: 18 \\
: 24\end{array}$ & $\begin{array}{l}88.0 \\
83.0 \\
25.0 \\
22.0 \\
22.0 \\
22.0 \\
21.0 \\
21.0 \\
21.0 \\
22.0 \\
22.0 \\
44.0 \\
51.0 \\
88.0 \\
90.0 \\
96.0 \\
30.0\end{array}$ & 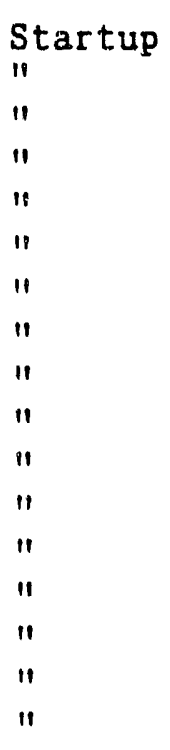 \\
\hline
\end{tabular}




\section{CITY WATER, IIGIT \&: POWER}

3100 steveneob dxive

\section{UNII OPACUTY CDM}

DOWNTIMAS RUPORI

Unit: Lakeside

Month/Year: June 1992 


\section{CITY WATER, TIGIT \& POWPR \\ 3100 stevenson Dxive. \\ FXCISS OPACITY REPORT}

Jnit: Lakeside

Month/Year: June 1992

Opacity Limit: $30 \%$
BOILER HOURS

Unit 非 - 199

Unit $\$ 8-141$

ay Start Opacity $\%$ Cause Corrective Action Taken

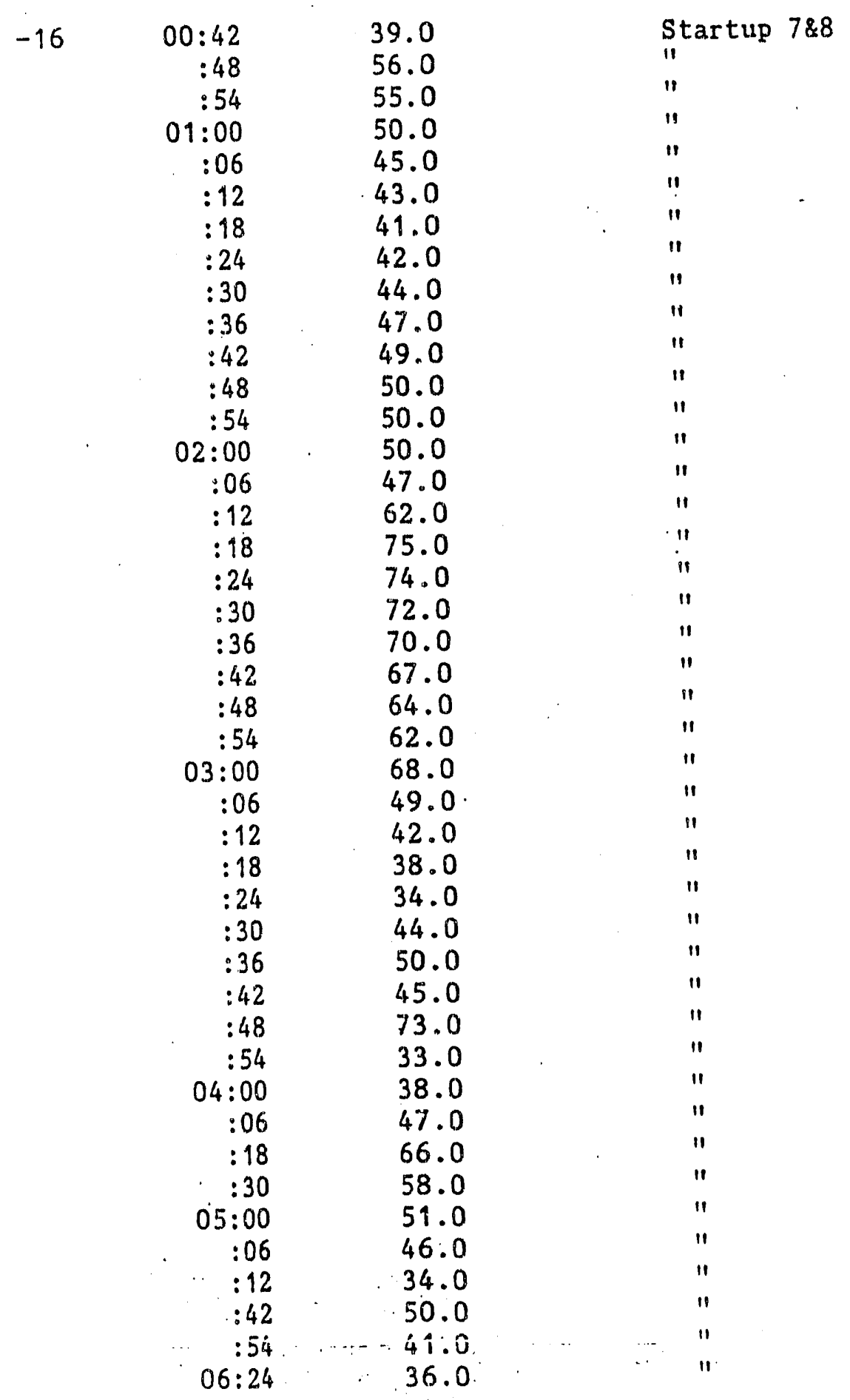


cess Opadity Report

keside - June 1992

ge -2-

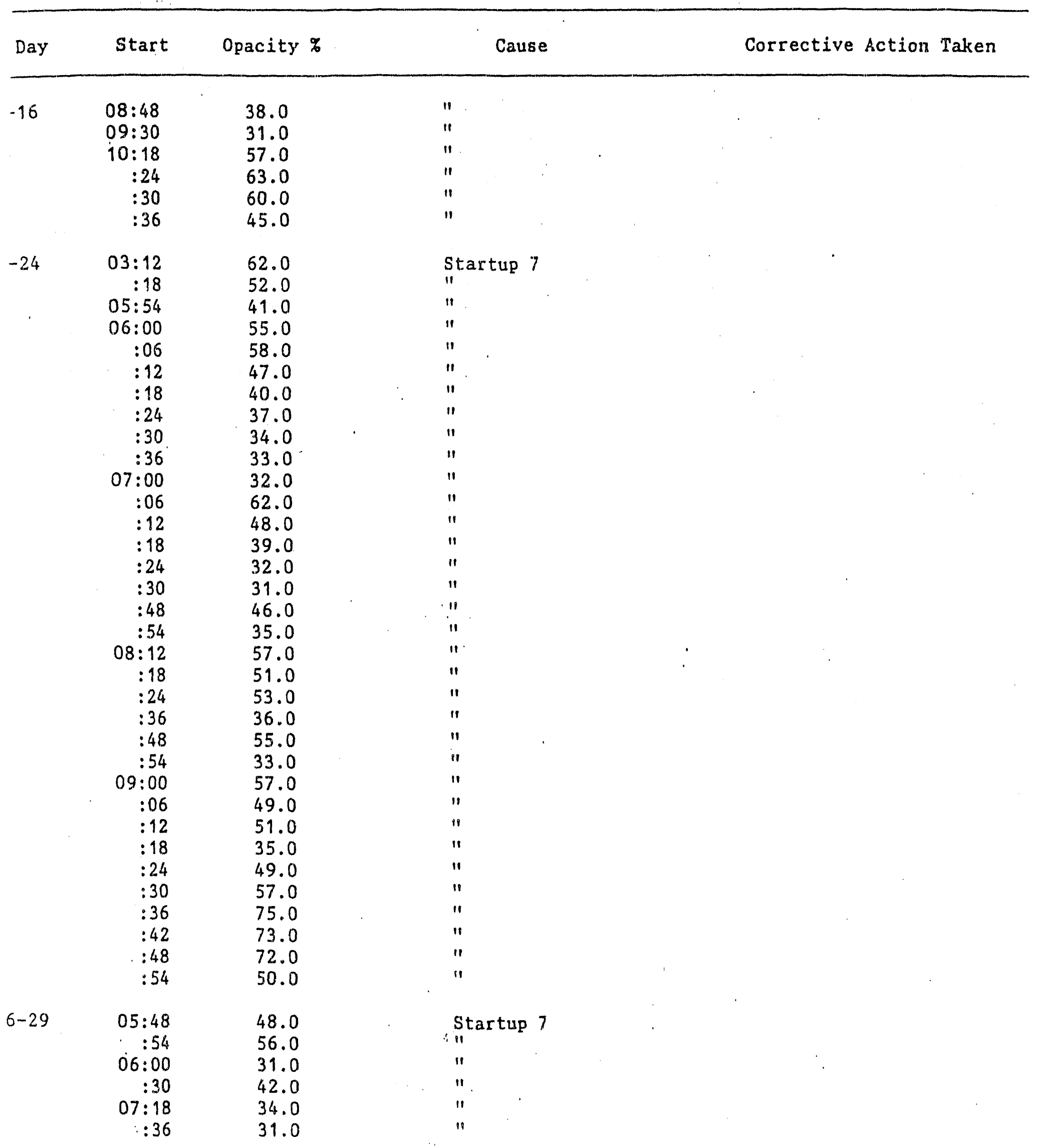


xcess Opąity Report

akes1de - June 1992

age -3-

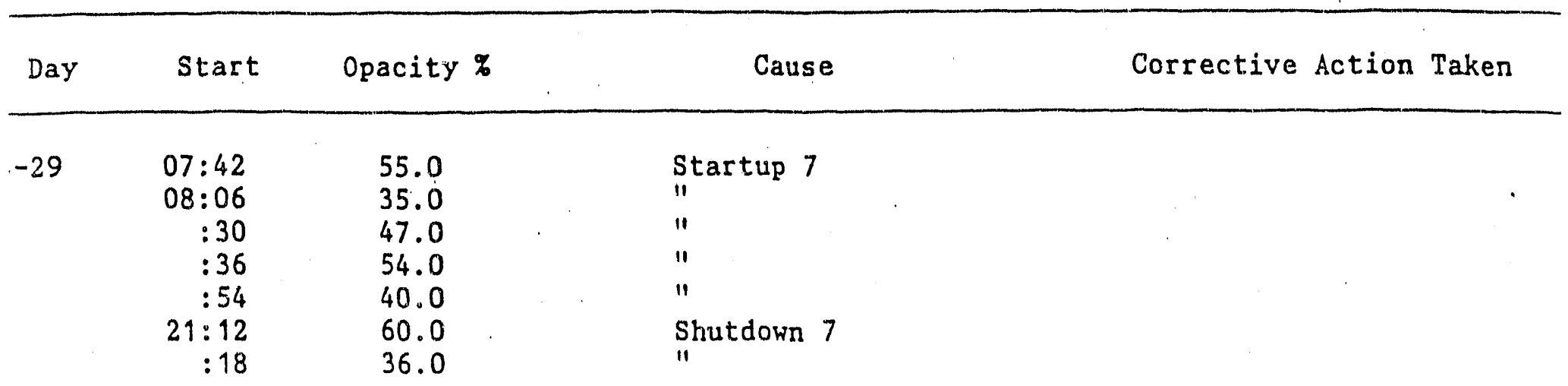




\section{CITY WATER, IIGEII \& POWNR}

3100 stevensol Dxive

\section{UNII OPACITY CIM}

DOWNIIME REPORT

\section{Unit: $\quad 31$}

Month/Year: June 1992 


\title{
CIIY WAWIER IIGEIE \& POWER \\ 3100 stevenson Drive \\ FXCBSS OPACITY TREPORT
}

\begin{abstract}
Unit: $\quad 31$
Month/Year: June 1992

Opacity Limit: $30 \%$
\end{abstract}

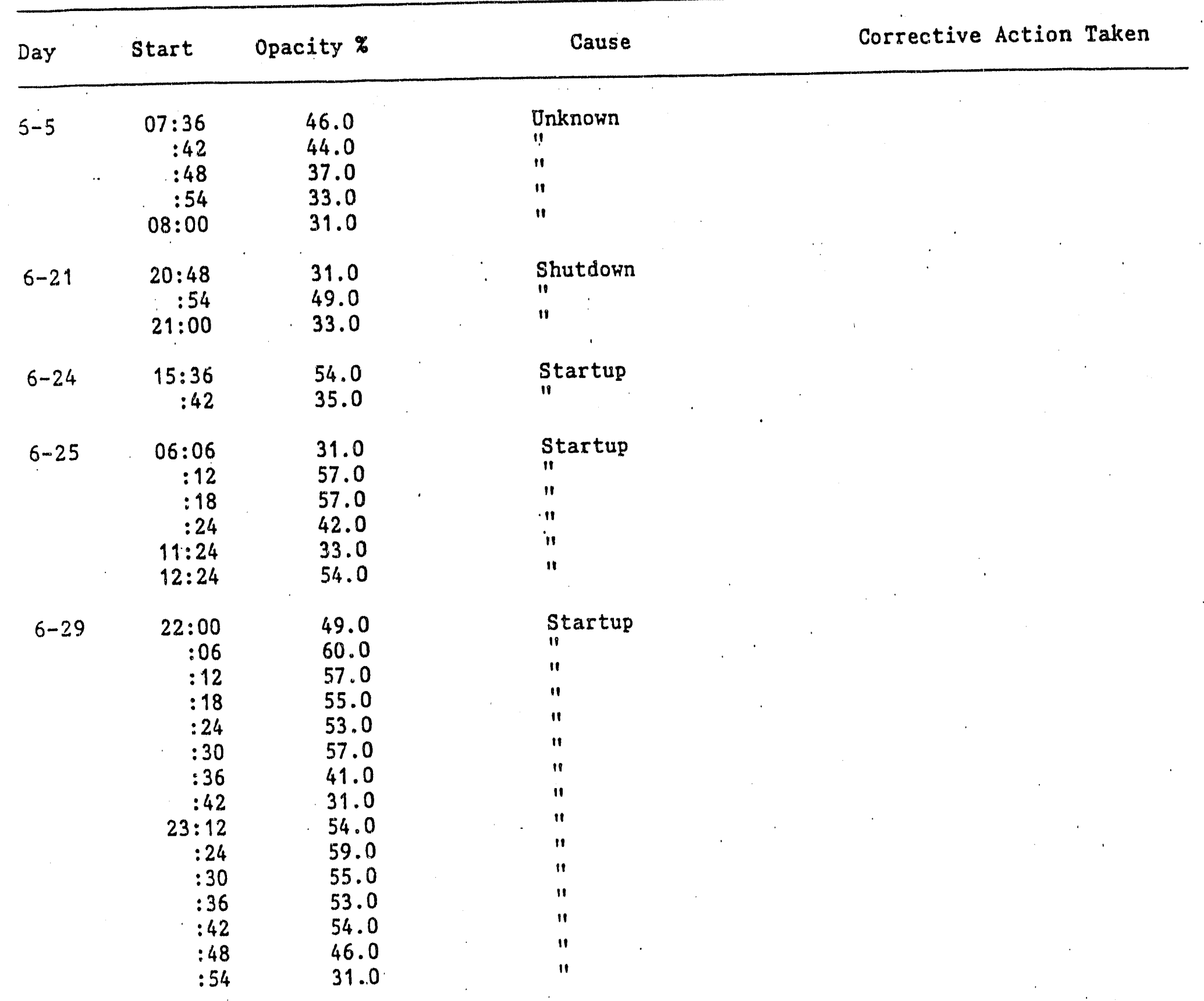


Excess Opacity Report

Unit 31 - June 1992

Page -2--

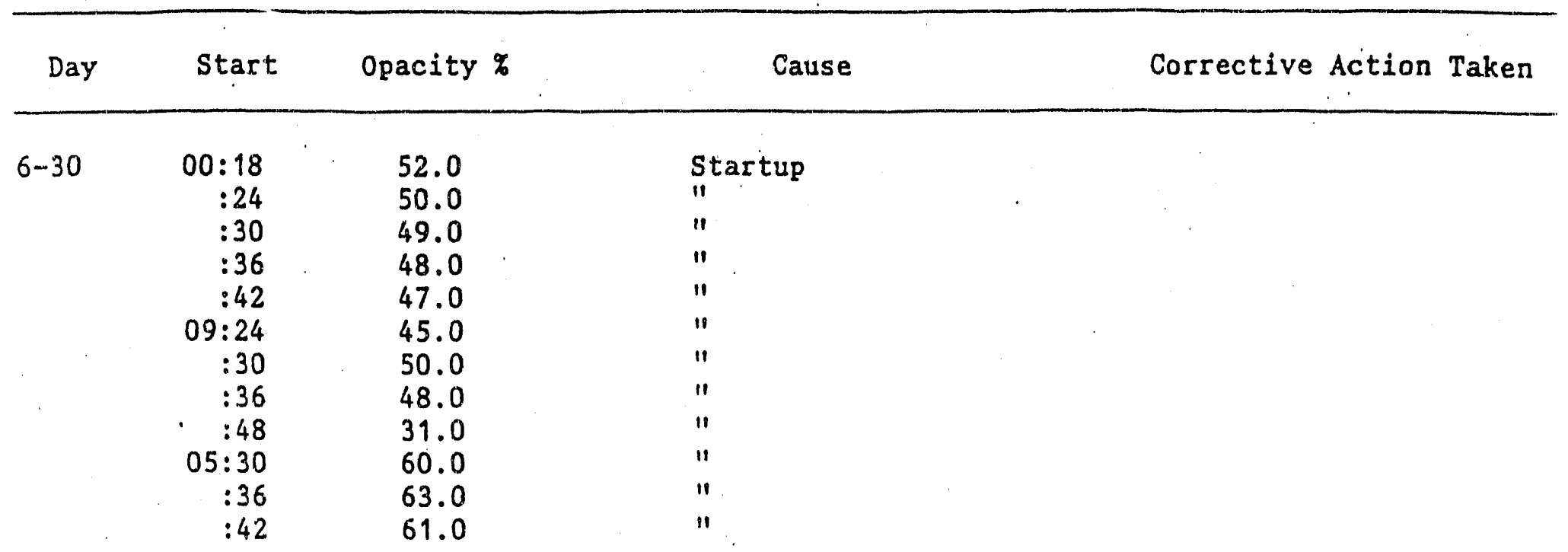




\section{CIIY WATIER, IIGFT \& POWTER}

3100 stevenaor Drive

UNIx OPRCITY CWM

DOWNTIMIS IREPOIRI

Unit: $\quad 32$

Month/Year: June 1992

Day Start-End Component Cause Corrective Action Taken

NONE 


\section{CITY WATER: IIGET \& POWDER \\ 3100 stevenaon Dxive \\ FXCESS OPACITY RTPORT}

Unit: $\quad 32$

Month/Year: June 1992

Opacity Limit: $30 \%$

\begin{tabular}{|c|c|c|c|c|}
\hline Day & start & Opacity \% & Cause & Corrective Action Taken \\
\hline
\end{tabular}

$\begin{array}{rrrl}6-2 & 14: 36 & 42.0 & \text { Startup } \\ : 42 & 40.0 & " 1 \\ : 48 & 41.0 & " 1 \\ : 54 & 36.0 & "\end{array}$

\begin{tabular}{|c|c|c|c|}
\hline $6-5$ & $\begin{array}{r}06: 36 \\
: 42 \\
07: 12 \\
: 18 \\
:: 24 \\
: 30 \\
: 36 \\
15: 00 \\
: 06 \\
: 12 \\
: 18\end{array}$ & $\begin{array}{l}42.0 \\
42.0 \\
45.0 \\
52.0 \\
61.0 \\
56.0 \\
46.0 \\
55.0 \\
79.0 \\
79.0 \\
35.0\end{array}$ & $\begin{array}{l}\text { Shutdown } \\
\text { " } \\
\text { " } \\
" 1 \\
" 1 \\
" 1 \\
" 1 \\
" 1 \\
\text { " }\end{array}$ \\
\hline
\end{tabular}




\section{CIYY WATER, IIGIII \& POWDR \\ 3100 stevensom Dxive \\ UNIT OPACIMY CMM \\ DOWNTIME IREORT}

Unit: $\quad 33$

Month/Year: June 1992

Day Start-End Component $\quad$ Cause Corrective Action Taken

$\begin{array}{llll}6 / 22 & 09: 00-16: 00 & \text { 0pacity Recorder } & \text { Stuck in Cal. } \\ 6 / 26 & 00: 000 & & \text { Malfunctioning } \\ 6 / 29 & -10: 00 & \text { Transmissometer } & \end{array}$

Replaced Board 


\section{CIIY WATER, IIGKIY \& POWDER \\ 3100 steveman Drive}

\section{FXCESS OPACITY REPORT}

it: $\quad 33$

nth/Year: June 1992

acity Limit: $30 \%$

Start Opacity \%

Cause

Corrective Action Taken

\begin{tabular}{|c|c|c|}
\hline $\begin{array}{r}00: 06 \\
: 12 \\
: 18 \\
: 24 \\
: 30 \\
: 36 \\
: 42 \\
: 48 \\
: 54 \\
01: 00 \\
: 06 \\
: 12 \\
: 18 \\
: 24 \\
: 30 \\
: 36 \\
: 42 \\
: 48 \\
: 54 \\
02: 00 \\
: 06 \\
: 12 \\
: 18 \\
: 30 \\
: 36 \\
: 42 \\
: 48 \\
: 54 \\
03: 00 \\
: 06 \\
: 12 \\
: 18 \\
: 24 \\
: 30 \\
: 36 \\
: 44 \\
: 48 \\
: 54\end{array}$ & $\begin{array}{l}21.0 \\
21.0 \\
21.0 \\
21.0 \\
21.0 \\
22.0 \\
22.0 \\
23.0 \\
23.0 \\
23.0 \\
23.0 \\
23.0 \\
24.0 \\
24.0 \\
24.0 \\
24.0 \\
24.0 \\
24.0 \\
24.0 \\
24.0 \\
24.0 \\
24.0 \\
24.0 \\
24.0 \\
35.0 \\
25.0 \\
25.0 \\
25.0 \\
24.0 \\
25.0 \\
25.0 \\
25.0 \\
25.0 \\
25.0 \\
25.0 \\
25.0 \\
25.0 \\
25.0\end{array}$ & $\begin{array}{l}\text { The load had dropped } \\
\text { after boiler adjust- } \\
\text { ments, the opacity } \\
\text { alarms did not seem } \\
\text { to be functioning. } \\
\text { Therefore, the Operator } \\
\text { did not realize the } \\
\text { opacity had exceeded } 20 \%\end{array}$ \\
\hline
\end{tabular}

The procedures have been changed for the Operators to inspect the opacity strip charts. 
cess Opacity Report

it 33 - June 1992

ge -2 -

\begin{tabular}{|c|c|c|c|c|}
\hline Day & start & Opacity $\%$ & Cause & Corrective Action Taken \\
\hline
\end{tabular}

\begin{tabular}{|c|c|c|c|}
\hline $\begin{array}{r}04: 00 \\
: 06 \\
: 12 \\
: 18 \\
: 24 \\
: 30 \\
: 36 \\
: 42 \\
: 48 \\
: 54 \\
05: 00 \\
: 06 \\
: 12 \\
: 18 \\
: 24 \\
: 30 \\
: 36 \\
: 42 \\
: 48 \\
: 54 \\
06: 06 \\
: 12 \\
: 24 \\
: 30 \\
: 36 \\
: 42 \\
: 48 \\
07: 00\end{array}$ & $\begin{array}{l}25.0 \\
25.0 \\
25.0 \\
25.0 \\
25.0 \\
25.0 \\
25.0 \\
25.0 \\
25.0 \\
25.0 \\
25.0 \\
25.0 \\
25.0 \\
25.0 \\
26.0 \\
26.0 \\
24.0 \\
24.0 \\
24.0 \\
22.0 \\
22.0 \\
22.0 \\
22.0 \\
22.0 \\
21.0 \\
22.0 \\
29.0 \\
21.0\end{array}$ & $\begin{array}{l}\text { The load had dropped } \\
\text { after boiler adjust- } \\
\text { ments, the opacity } \\
\text { alarms did not seem } \\
\text { to be functioning. } \\
\text { Therefore, the operator } \\
\text { did not realize the } \\
\text { opacity had exceeded } 20 \%\end{array}$ & $\begin{array}{l}\text { The procedures have been } \\
\text { changed for the Operators } \\
\text { to inspect the opacity } \\
\text { strip charts. }\end{array}$ \\
\hline $\begin{aligned} 17 & : 42 \\
: & : 48 \\
18 & : 00\end{aligned}$ & $\begin{array}{l}23.0 \\
23.0 \\
22.0\end{array}$ & $\begin{array}{l}\text { Precip. Malfunction } \\
" 1\end{array}$ & $\begin{array}{l}\text { Reset field } \\
\text { " } \\
\text { " }\end{array}$ \\
\hline
\end{tabular}

\begin{tabular}{|c|c|c|}
\hline $\begin{array}{r}05: 00 \\
: 06 \\
: 12 \\
: 24 \\
: 30 \\
: 36 \\
: 42 \\
: 48 \\
: 54 \\
06: 00 \\
: 06 \\
: 12 \\
: 18 \\
: 24 \\
: 30 \\
: 36\end{array}$ & $\begin{array}{l}65.0 \\
65.0 \\
88.0 \\
63.0 \\
63.0 \\
22.0 \\
22.0 \\
61.0 \\
61.0 \\
66.0 \\
66.0 \\
63.0 \\
63.0 \\
73.0 \\
73.0 \\
61.0\end{array}$ & 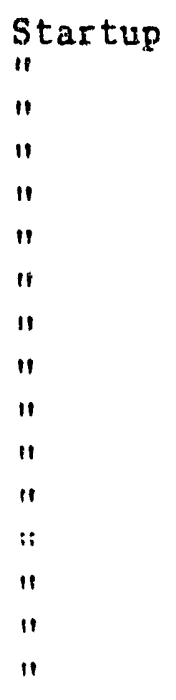 \\
\hline
\end{tabular}


sess Dpacity Report

it 33 - June 1992

ie $-3-$

Day Start Opacity $\% \quad$ Cause $\quad$ Corrective Action Taken

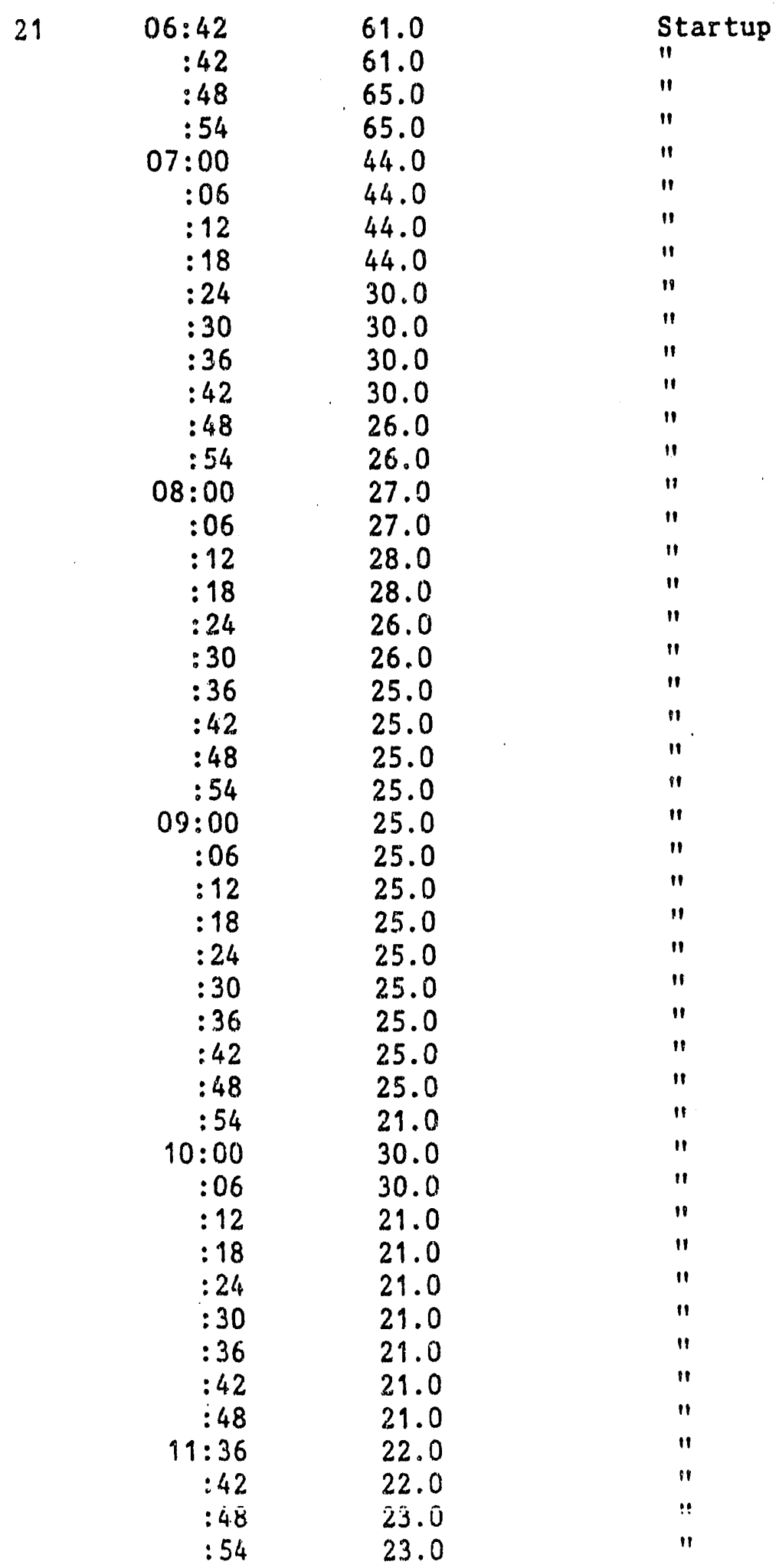


zess Qpacity Report

it 33 - June 1992

se $-4-$

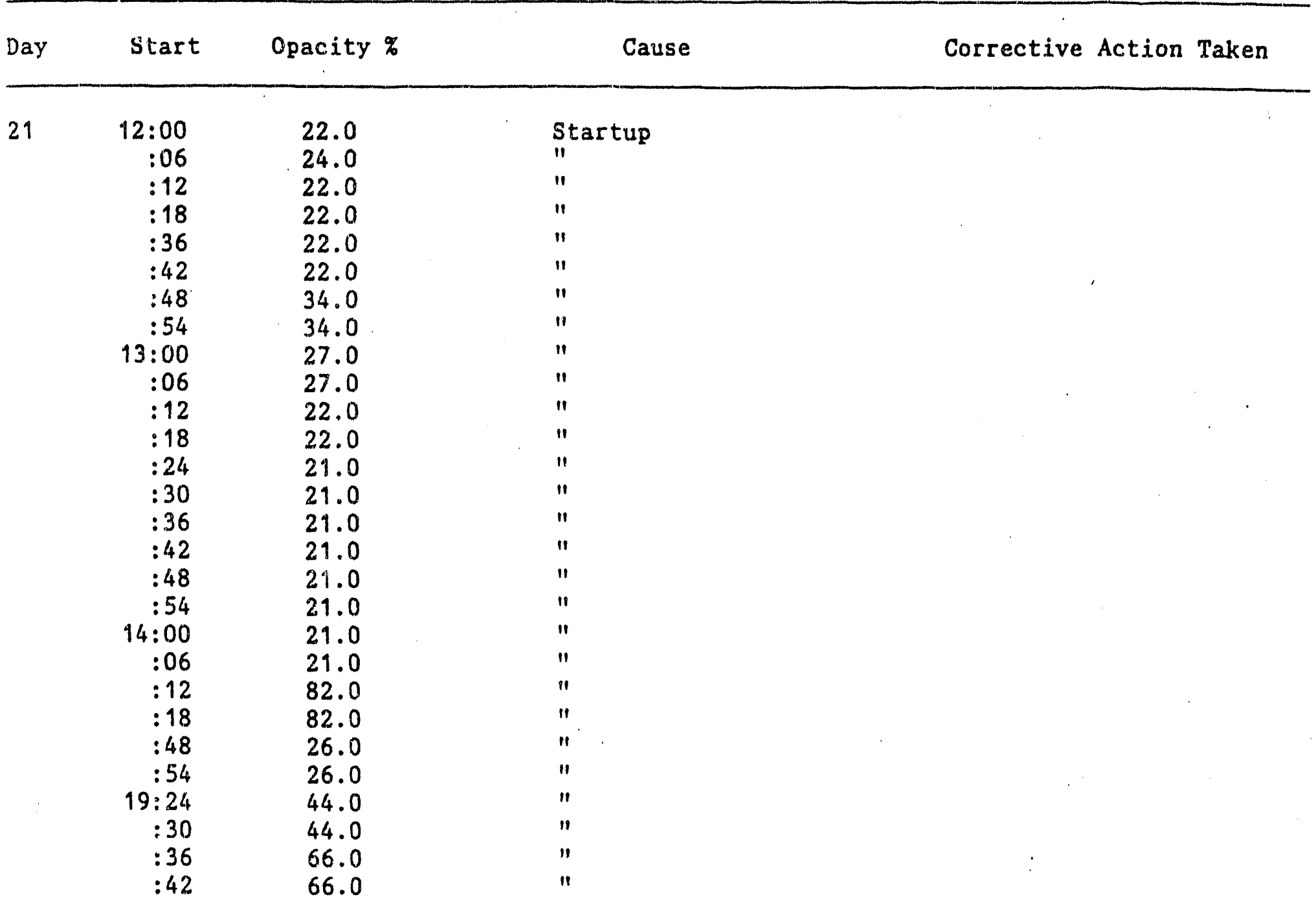


APPENDIX C 
NPOES Permit No. ILO001554

Illinois Environmental Protection Agency

Division of Hater Pollution Control

2200 Churchill Road

P.O. Box 19276

Springfield, Illinois 62794-9276

NATIONAL POLLUTANT DISCHARGE ELIMINATION SYSTEM

Reissued (NPOES) Permit

Expiration Date: July 1, 1997

Name and Address of Permittee:

Illinois Power Company

500 South 27 th Street

Post Office Box 511

Decatur, Illinois 62525-1805

Discharge Number and Name:

No. 001 Condenser Cooling Water

No. DOI(a) Boiler 81 lowdown

No. 001 (b) Intake Screen Backwash

No. D0I(c) Roof Drain Discharge

No. 003 Ash Lagoon \#2 and \#4 Discharge

No. 005 Ash Lagoon $\# 9$ and $\$ 3$ Discharge

No. 005(a) Chemical Metal Cleaning Waste Treatment System Effluent

In compliance with the provisions of the IlTinois Environmental Protection Act, Subtitle C RuTes and Regulations of the Illinois Pollution Control Board, and the FWPCA, the above-named permittee is hereby authorized to discharge at the above location to the abovemamed receiving strean in accordance with the standard conditions and attachments herein.

Permittee is not authorized to discharge afker the above expiration date. In order to receive authorization to discharge beyond the expiration date, the permittee shall submit the proper application as required by the 111 inois Environmental Protection Agency (IEPA) not 1 ater than 180 days prior to the expiration date.
Issue Date:

Effective Date:

Facility Name and Address:

Illinois Power Company

Hennepin Power Plant

Hennepin, Illinois 61327

Putnam County

Receiving Haters

Illinois River
Thomas G. MeSwiggin, P.E.

Manager, Permit Section

Division of Hater Pollution Control

TGM: TRK: $j d / 0495 E / 3$ 
Page 2

NPDES Permit No. ILO001554

Effluent Limitations and Monitoring

\begin{tabular}{|c|c|c|c|c|}
\hline & $\begin{array}{l}\text { LOAO LIMITS } \\
\text { Ibs/day }\end{array}$ & $\begin{array}{c}\text { CONCE } \\
\text { LIN }\end{array}$ & $\begin{array}{l}\text { TION } \\
\text { mg } / 1\end{array}$ & \\
\hline RAMETER & $\begin{array}{ll}30 \text { DAY } & \text { DAILY } \\
\text { AVG. } & \text { MAX. }\end{array}$ & $\begin{array}{l}\text { 30 OAY } \\
\text { AVG. }\end{array}$ & $\begin{array}{l}\text { DAILY } \\
\text { MAX. }\end{array}$ & $\begin{array}{l}\text { SAMPLE } \\
\text { FREQUENCY }\end{array}$ \\
\hline
\end{tabular}

1. From the effective date of this permit until the expiration date, the effluent of the following discharge(s) shall be monitored and limited at all times as follows:

Outfall(s): 001 Condenser Cooling Water

This discharge consists of:

1. Condenser Cooling Water

2. House Service Water

3. Boiler Blowdown

4. Intake Screen Backwash

5. Roof Drain Discharge

Flow

Approximate Flow

$153.26 \mathrm{MGD}$

7.0 MGO

0.027 MGD

$0.258 \mathrm{MGO}$

Intermittent

$\begin{array}{ll}\text { Daily } & 24 \text { Hour } \\ \text { Total }\end{array}$

Total Residual

Chlorine See Special Condition No. 3

INeek *

Temperature See Special Condition No. 4

Daily Continuous

"See Special Condition No. 3

Outfall(s): 001(a) Boiler Blowdown

Approximate Flow 0.027 MGD

Flow

iNeek

Single

Reading-

Estimate

Total Suspended Solids

15.0

30.0

INeek

24-Hour Composito 
Page 3

NPOES Permit No. ILO001554

Effivent Limitations and Monitoring

\begin{tabular}{|c|c|c|c|c|c|c|}
\hline & \multicolumn{2}{|c|}{$\begin{array}{l}\text { LOAD LIMITS } \\
\text { Ibs/day }\end{array}$} & \multicolumn{2}{|c|}{$\begin{array}{l}\text { CONCENTRATYON } \\
\text { LIMITS ma/I }\end{array}$} & \multirow[b]{2}{*}{$\begin{array}{l}\text { SAMPLE } \\
\text { FREQUENCY }\end{array}$} & \\
\hline ARAMETER & $\begin{array}{l}30 \text { DAY } \\
\text { AVG. }\end{array}$ & $\begin{array}{l}\text { DAILYY } \\
\text { MAX. }\end{array}$ & $\begin{array}{l}30 \text { DAY } \\
\text { AVG. }\end{array}$ & $\begin{array}{l}\text { DAILY } \\
\text { MAX. }\end{array}$ & & $\begin{array}{l}\text { SAMPLE } \\
\text { TYPE }\end{array}$ \\
\hline
\end{tabular}

1. From the effective date of this permit until the expiration date, the effluent of the following discharge(s) shall be monitored and limited at all times as follows:

Outfall(s): 001(b) Intake Screen Backwash

Approximate Flow

0.26 MGD

Flow

$\begin{array}{ll}\text { Wheek } & \text { Single } \\ \text { Reading } \\ \text { Estimate }\end{array}$

So as to minimize adverse impacts, for purposes of this permit, the intake structure operation and maintenance shali include, but not be limited to, the following:

1. Outer bar racks shall be routinely cleaned

and collected debris properly disposed.

2. The traveling screens shall commence operating whenever the head loss across

the screens exceeds four (4) inches.

3. The traveling screens shall be operated at least once per $a$ hour shift, provided, however, that this requirement shall be inapplicable when the generating units are not operating.

Outfall(s) $001(c)$ Roof Drain Discharge

This discharge collsists of:

1. Power Block Building Roof Orains

Approximate Flow

See Standard Condition No. 17 
Page 4

NPDES Permit No. ILO001554

Effluent Limitations and Monitoring

PARAMETER

LOAO LIMITS
IbSLDQY OAILY

CONCENTRATION
$\frac{\text { LAMITS mG/I }}{30 \text { DAY DAILY }}$

SAMPLE

SAMPLE

AVG. MAX.

AVG.

FREQUENCY TYPE

1. From the effective date of this permit until the expiration date, the effluent of the following discharge(s) shall be monitored and limited at all times as follows:

Outfal1(s): 003 Ash Lagoon \#2 and \#4 Discharge

This discharge consists of:

1. Unit 2 Bottom Ash and Fly Ash

2. Demineralizer Regenerate Wastes

3. Unit 2 Non-chemical Metal Cleaning Washwater

4. Unit \#1 and Unit \#2 Ash Hopper Overflow"

5. Fly Ash Air Separator Overflow

6. Ash Hopper Tank Emergency Overflow

7. Demineralizer Room Floor Drainage

8. Power Block Building Floor Drains and Sump Discharges

9. Reverse Osmosts Unit Concentrate

Approximate flow
2.9 HGD
0.035 MGD
Intermittent
0.20 HGD
Intermittent
Intermittent
Intermittent
0.1 MGD
0.036 MGD

Flow

See Special Condition No. 1

ineak

pH

Week Grab

Total Suspended Solids

$0 i 1$ and Grease
15.0

15.0

"This wastestream may be discharged to the East (Outfal System.

"-See Special Condition No. 6 
Page 5

NPDES Permit No. ILOOO1554

\section{DRAFT}

Effluent Limitations and Monitoring

\begin{tabular}{l} 
LOAD LIMITS \\
Ibs/daY \\
\hline 30 DAY \\
AVG.
\end{tabular}

$\begin{gathered}\text { CONCENTRATION } \\ \text { LIMIIS mo/I }\end{gathered}$
$\frac{\text { OAILY }}{30 \text { OAY }}$
AVG.

SAMPLE
FREQUENCY SAMPLE
TYPE

PARAMETER

AVG.

AVG.

1. From the effective date of this permit until the expiration date, the effluent of the following discharge(s) shall be monitored and limited at all times as follows:

Outfall(s): 005 Ash Lagoon $\# 1$ and $\# 3$ Discharge

This discharge consists of:

1. Unit \#1 Bottom Ash and Fly Ash Transport Water

2. Unit \#1 and \#2 Ash Hopper Overflow"

3. Coal Breaker Building Drain Sump

4. Illinois River Dredge Spoils

5. Unit $\# 1$ Non-chemicai Metal Cleaning Washwater

6. Chemical Metal Cleaning Waste Treatment System Effivent

7. Coal Pile Runoff

8. Unit $\# 1$ and Unit \#2 Ash Line Low Point Drainoff

9. Crib House Sump

10. Well Water Orain Line

11. Water Treatment Plant Sump

12. Boiler Orum Chemical Tank Orainage

13. Gas Reburning/Sorbent Injection Waste

Flow

pH

See Special Condition No. I

$$
\begin{aligned}
& \text { Approximate Flow } \\
& 0.21 \text { MGD } \\
& 0.2 \text { MGD } \\
& \text { Intermittent } \\
& \text { Intermittent } \\
& \text { Intermittent } \\
& \text { Intermittent } \\
& \text { Intermittent } \\
& \text { Intermittent } \\
& \text { Intermittent } \\
& \text { Intermittent } \\
& \text { Intermittent } \\
& \text { Intermittent } \\
& 0.96 \mathrm{HGD}
\end{aligned}
$$

\begin{tabular}{|c|c|}
\hline INeek & $\begin{array}{l}\text { Singie } \\
\text { Reading } \\
\text { Estimate }\end{array}$ \\
\hline INeek & Grab \\
\hline INeek & $\begin{array}{l}24 \text { Hrninm } \\
\text { Composite }\end{array}$ \\
\hline $2 /$ Month & Grab \\
\hline
\end{tabular}




\section{DRAFT}

Page 6

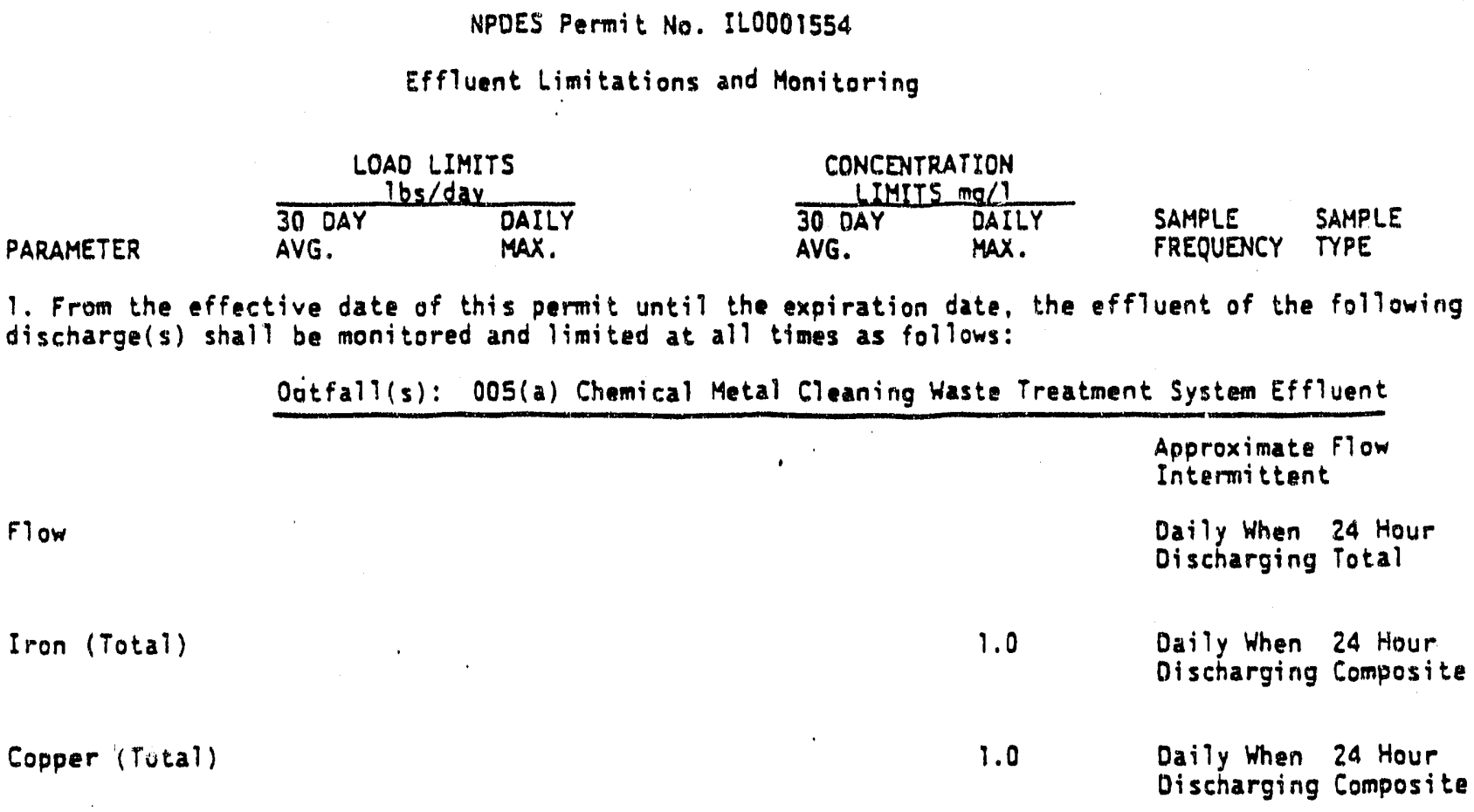


NPOES Permit No. ILOOOI554

\section{Soecial Conditions}

1. The $\mathrm{pH}$ shall be in the range 6.0 to 9.0 .

2. Samples taken in compliance with the ffluent monitoring requirements shall be taken at a point representative of the discharge, but prior to entry into the receiving strieam.

3. A. During times when the condenser cooling water is chlorinated intermittently, botal residual chlorine (TRC) may not be discharged from the station for more than three hours per day or from any single generating unit's main cooling condensers for more than two hours per day. The discharge limit is $0.2 \mathrm{mg} / 1$, measured as an instantaneous maximum.

1. Continuous TRC monitoring throughout a representative chiorination period shall be performed once per week in the cooling water discharge flum during the respective chlorination period of one condenser half allowing for lag time between the initiation of chlorination and the time of sampling. For continuous chlorine monitoring, analytical data from only two representative monitoring periods each month need be reported on the monthly discharge monitoring report. The time of sampling, the time and duration of the chlorine dosing beriod, and the amount of witine applind shall be reported.

2. If continuous monitoring cannot be performed, minimum of three grab samples shall be collected in the discharge flume at five minute intervals or lass, once per week during a representative chlorination period, allowing for lag time between the initiation of chlorination and the time of sampling. to develop a chlorine concentration curve. The time of sampling, the time and duration of the chiorine dosing period, and the amount of chlorine applied shall be reported.

B. During times when the condenser cooling water is chiorinaped continuousiy, the discharge litmit is $0.05 \mathrm{mg} / \mathrm{l}$, measured as an instantaneous maximum.

C. Chlorination of house service water is atthorized by this permit, provided that the discharge limits above are not exceeded at Outfall 001.

4. Discharge of wastewater from this facility must not alone or in combination with other sources cause the receiving stream to violate the following themal limitations at the edge of the mixing zone which is defined by Section 302.211. Iliinois Administrative Code, Title 35. Chapter 1, Subtitle C, as amended:

A. Maximu temperature rise above natural temperature inst not exeed $5 \circ f\left(2.710^{\circ} \mathrm{C}\right)$.

B. Water temperature at representative Tocations $i$. the main river shall not exceed the maximum limits in the following table during more than one (1) percent of the hours in the 12 month period ending with any month. Moreover, at no time shall the water temperature at such locations exceed the maximum limits in the following table by more than $3 \circ \mathrm{F}(1.67 \circ \mathrm{C})$. (Main river temperatures are temperatures of those portions of the river essentially similar to and following the same themal regims as the temperatures of the main flow of the river.)

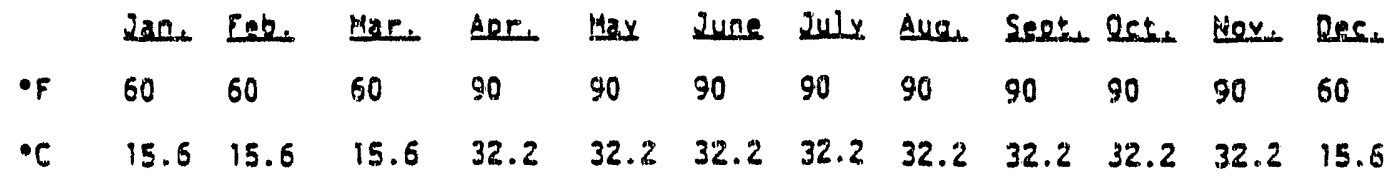

C. At times when the river water intake temperature is less than or equal to $4 l^{\circ} \mathrm{F}$, the distharge temperature shali not axceed t! intake temperature by more than $18 \circ \mathrm{F}$.

5. Thare shall be no discharge of polychlorinated biphenyl compounds.

6. If incizment wecther prohibits the collection of a 24 hour composite sample for five consecutive days, sanpling shall consist of a grab samole.

7. Illinois Power Company has comolied with Section $302.211(f)$ of iitle 35. Chapter 1, Subtitle C: Hater Pollutien Regulations and Section $316(a)$ of the Cha by bemonstrating that thermal discharge from Hennepin power plant has nob caused and cannoq reasonably be expected lo cause significant ecological danace to the dllinois River as aonroves by the iPCB in PCB $78-3$ on 
Page 8

NPOES Permit No. IL0001554

\section{Soecial Conditions}

8. Illinois Power Company's demonstration for the Hennepin Power Plant in accordance with Section $316(6)$ of the CWA has been approved by this Agency by letter dated December 29, 1978. It is determined that no additional intake monitoring or modification is being required for reissuance of this NPDES permit.

9. Standard Condition No. S shall not constitute a waiver of any constitutional rights of the permittee.

10. The provisions contained in Standard Condition No. 17 shall not prejudice permittee's right to obtain or be granted a reasonable time in which to comply, but in no event strail such time be later than any applicable federal or State of Illinois statutory or regulatory compliance date, in connection with any modification made pursuant thereto.

11. Standard Condition $11(a)$ of Attachment $H$ is rewritten as follows:

An application submitted by a corporation shall be signed by a principal executive officer of at least the level of vice president, or his duly authorized representative. if such representative is responsible for the overall operation of the facility from which the discharge deseribed in the application form originates. In the case of a partnership or a sole proprietorship, the application shall be signed by a general partner or the proprietor, respectively. In the case of a publicly owned faciliey, the apelication shall be signed by either the principal executive officer, ranking elected official, or other duly authorized employee.

12. Standard Condition $11(b)$ of Attachment $H$ is rewritten as follows:

Pursuant to 40 CFR $122.22(b)$ all reports required by perinits, other information requested by the Director, and all permit applications submitted for Group II storm water discharges under $122.26(b)(3)$ shail be signed by person described in 40 CFR 122.22 (a), or by a duly authorized representative of that person. A person is a duly authorized representative oniy if :

(1) The authorization is made in writing by a person described in paragraph (3) of this section:

(2) The authorization specifies either an individual or a position having responsibility for the overall operation of the regulated facility or activity such as the position of plant manager, operator of a well or a well field. superintendent, position of equivalent responsibility, or an individual or position having overall responsibility, or an individual or position having overall responsibility for environmental matters for the company. (A duly authorized rapresentative may thus be either a maned individual or any individual occupying naned position.) and

(3) The witten authorization is submitted to the Director.

13. Disposal of GR-SI Residues in the West Ash Pond System shall be monitored and limited in accordance with the following Best Management Practices Plan:

a) Authorization to dispose GR-SI Residues in the West Ash Pond System is granted only for the term of the demonstration project. The Agency shall be notified on the date the demonstration project begins.

b) The raw residu eransport water shall be of adjusted to approximately 9.0 prior to discharge into the West. Ash Fond System using carbon dioxide, acetic acid or other approved chemicals which will not contribute chlorides or sulfates to the wastestream.

c) Groundwater moritoring shell be conducted during the denonstration profect and far six membs theresftel. Quarterly Iampling of all four existing downgradient wells for Boron, Cicium. Chioride. Manganese. Nitrate. Nitrite, PH, Sulfate, Sulfite and Total Dissolved Solids is required. Analytical data shall be submitted to the Agency within 60 days after sample collection. The first samples shall be collected upon conemencement of the demonstration project.

d) Modeling or dye tracing to identify the West Ash Pond System effluent mixing characteristics with the Illinois kiver shall be conducico and the point in the river where the sulfate water quality standard will be met identified. This information sha? be submitid to the Agency within six monehs after commencement of the demonstration 
NPDES Permit No. ILODO1554

Serecial Conditions

14. Sludge generated by the pretreatment of chemical metai cleaning waste in the chemical metal cleaning waste treatment tank at Hernepin Power Plant may be disposed of on the active area of the coal pile at the Hennepin Power plant within the following guidelines:

1. Chemical metal cleaning waste treatment tank sludge shall not exceed the Toxicity Characteristic regulatory levels when subjected to the Taxicity Characteristic Leaching Procedure for the 25 organic constituents and 8 metals regulated under the Toxicity Characteristic Rule (FR Vol. 55, No. 61/March 29, 1990, 11998-11877.

2. Sludge shall be applied on the active area of the coal pile.

3. Siudge shall be applied on the active area of the coal pile at a controlled rate to prevent coal pile runoff.

4. Sludge application shall not be permitted if the coal pile has been wetted by rainfall within the 24-hour period preceding the intended application time.

5. Sludge application shall not be permitted on the coal pile during precipitation or when precipitation is imminent.

6. The filter cake from trigo portable sock filter may be disposed on site with the sludge generated by the chemical netal cleaning wastewater treatment process.

7. Siudge or filter cake which is a hezardous waste shail not be piaced on the caal pile.

This Special Condition does not relieve the permittes of any State or faderal requirements for management of hazardous waste. Documentation to support a hazardous waste deturmination pursuant to $\triangle 0$ CFR 262.11 shall be maintained by the permittee.

15. The permittee shall record monitoring results on Discharge Monitoring Report Forms using one such form fur each discliarge each month.

The completed Discharge Monitoring Report forms shall be submitted to IEPA no later than the 15th day of the following month, unless otherwise specified by the permitting authority.

Discharge Monitoring Reports shall be mailed to the IEPA at the following addrass:

Illinois Environmental Protection Agency

Division of Water Poliution Control

2200 Churchill Road

Springfield, Illinois 62706

Attention: Compliance Assurance Section

16. Within 180 days after the beginning of discharge from the ash ponds (Outfalls 003 and 005 ), a 2 C application form shall be completed as required by 40 CFR $122.21(\mathrm{~g})(7)$ and submitted to IEPA. The monitoring must include at least three samples for the required metais, ammonia, and cyanide. This permit ma be modified. following public notice and opportunity for hearing, based on the monitoring results if necessary to prevent violations of water quality standards.

17. Ouring the time period of the GR-SI demonstration project, the following additional monitoring shall be done on Outfall 005 if discharge frow this outfall octura:

a. Chemical-specific Testing

On a quarterly basis, a grab sample of the discharge shall be analyzed for the following parameters, and the results reported with the oischarge Monitoring Report for the month

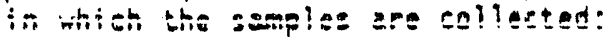

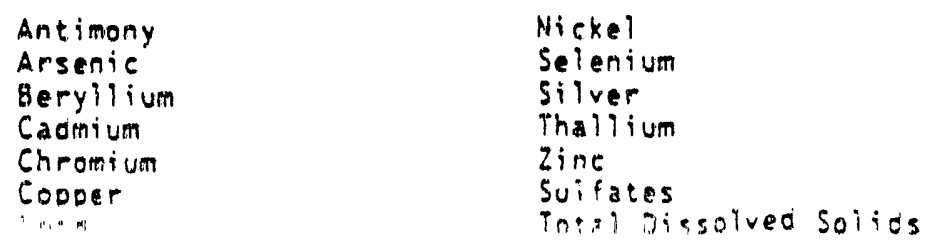


The permittee shall prepare a preliminary plan for biomonitoring and submit the plan to IEPA for review and approval within 90 days of the effective date of this permit. The pennittee shall begin biomonitoring of the effluent discharge within 90 days after approval of the biomonitoring plan or other such date as contained in the Agency's notification letter.

1. Acute. Toxicity - Standard definitive acute toxicity tests shall be run on two trophic leveis of aquatic species representative of the aquatic conmunity of the receiving strean. Except as noted here and in the IEPA document "Effluent Biomonitoring and Toxicity Assessinent." testing must be consistent with Hettods for Messuring the Acute Toxicity of Effluents to Aquatie Organisms EPA-500/4-85-013. Untess suostitute tests are pre-approved, the following tests are required:
a. Invertebrate 48 -hour static $L C_{50}$ Bioassay using Ceriodaphnia.
b. Fish 96-hour static renewal LC 50 Bioassay using fathead minnow.

2. Testing Frequency - The above tests stiall be conducted on a monthly basis for three months within 90 days following approval of the biomonitoring plan or other such date as contained in the Agency's notification (approval) letter. Results shall be reported according to EPA/600/4-85/014, Section 10, Report Preparation, and shall be submitted to IEPA within 1 week of becoming available to the permittee.

Should the results of any two months of sampling indicate toxicity for each month, the permittee shall submit within 90 days of the second toxicity event a plan for a toxicity identification evaluation (TIE) to the Agency. The Agency should be contacted at that time.

3. Toxicity Assessment - Should the review of the results of the biomonitoring program identify toxicity, the Agency may require that the pennittee prepare a plan for toxicity reduction evaluation and identification. This plan shall include an evaluation to determine which chemicals have a potential for being discharged in the plant wastewater, a monitoring program to determine their presence or absence and to identify other compounds which are not being removed by treatment, and other measures as appropriate. The permittee shail submit to the Agency its plan for toxicity reduction evaluation within 90 days following notifieation by the Agency. The permitse shall implement the plan within 90 days or other such date as contained in a notification letter received from the Agency.

The Agency may modify this permit during its term to incarporate additiond requirements or limitations bastd on the results of the biomonitoring. In addition, after review of the monitoring results, the Agency may modify this permit ta include numerical limitations for specific pollutants. Modifications under this condition shall follow public notice and opportunity for hearing.

c. Benthic and Sediment Monitoring

Within 90 days aftar the effective date of this permit, the permittee shall prepare a plan for benthic and sediment monitoring of the receiving stream in the imediate vicinity of Qutfall 005, and submit the plan to IEPA for review and approval. The monitoring program should be designed to document any instream chemical or biological impacts from the discharge, and should include monitoring prior to, during, and after discharges associated with the GR-SI project.

18. During the winter season (December I - March 15), a surmary of winter opepating conditions shall be prepared. The sumary shall include daily average and maximum "Oelta pr" and discharge temperatures. and shall be submitted to IEPA for review by May 15 of each year. 
$i$

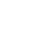
(2)

\section{ATTACHMENT H}

\author{
stevere Covertions
}

Delinierions

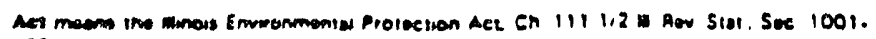
1051 a Amonoded

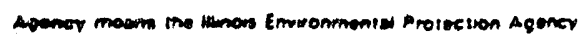

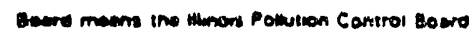

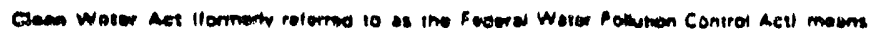

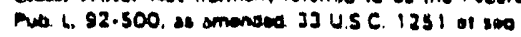

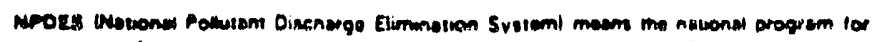

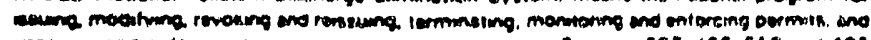

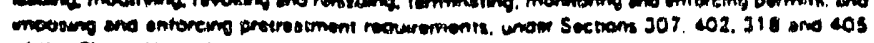
of the Claen wores act.

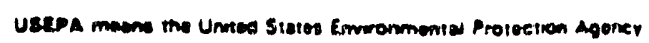

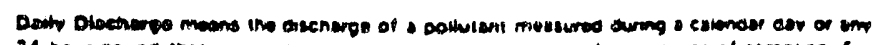

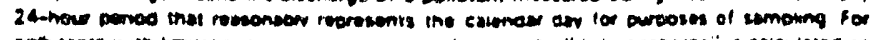

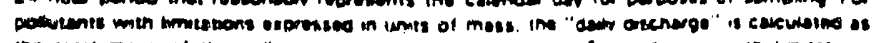

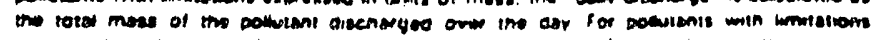

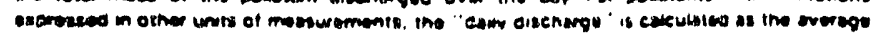

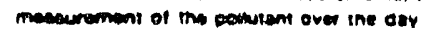

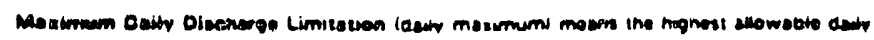
conomes.

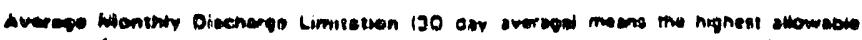

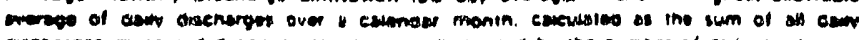

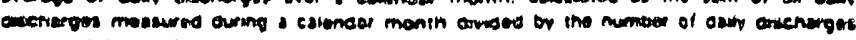
mosures ouns inot monin

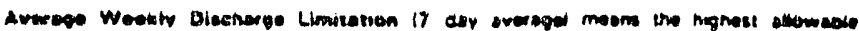

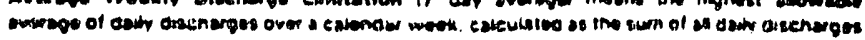

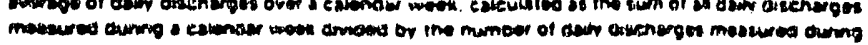
mat mont.

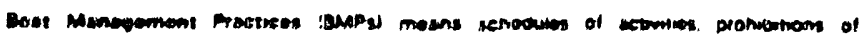

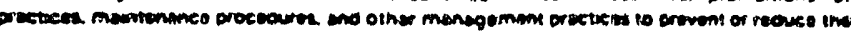

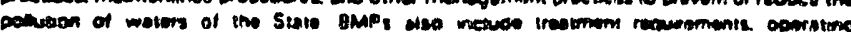

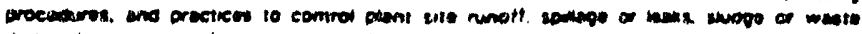

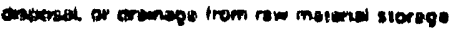

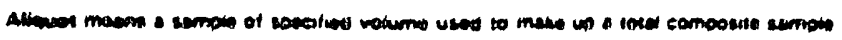

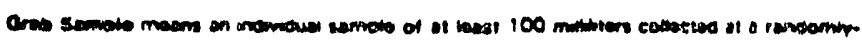

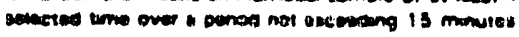

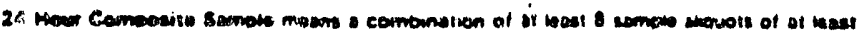

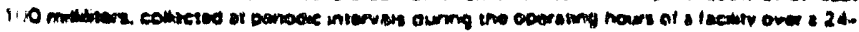
nour pourat

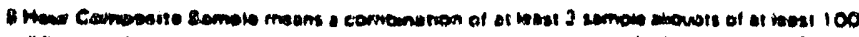

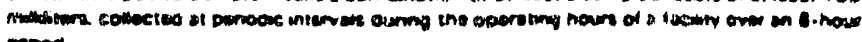
Denod

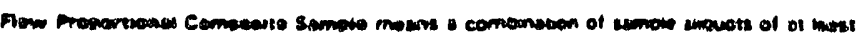

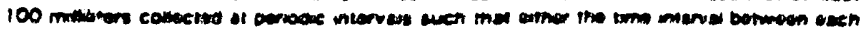

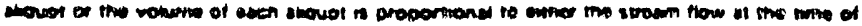

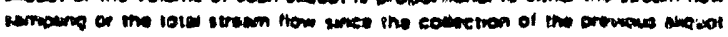

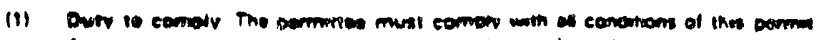

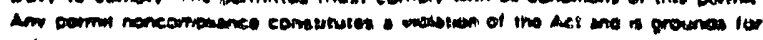

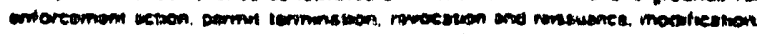

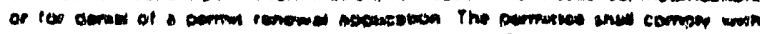

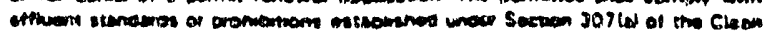

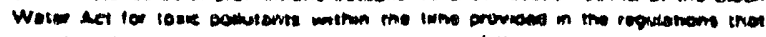

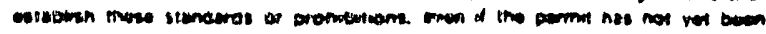

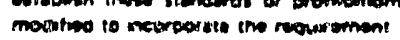

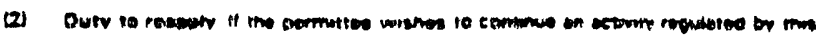

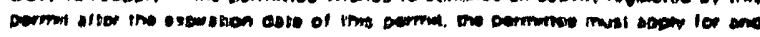

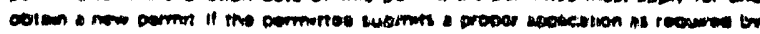

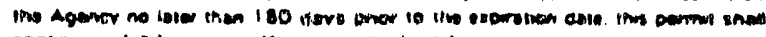

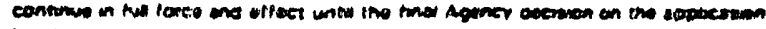
nim been mex

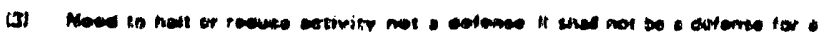

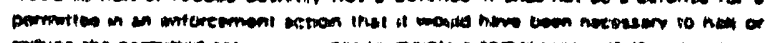

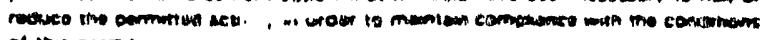
of ine dormin

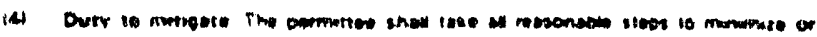

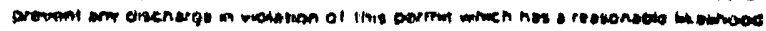

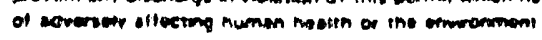

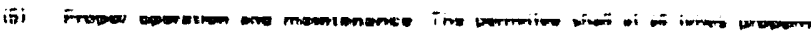

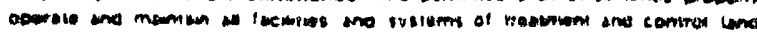

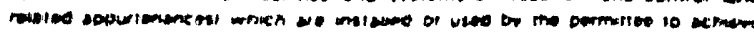

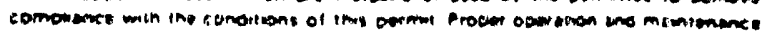

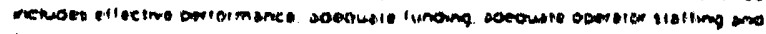

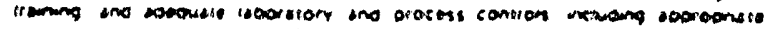

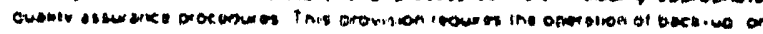

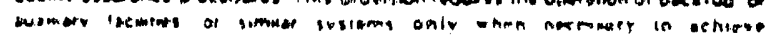

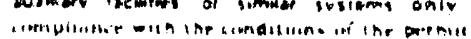

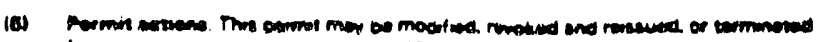

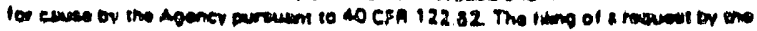

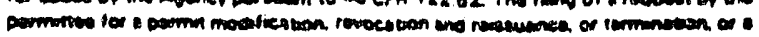

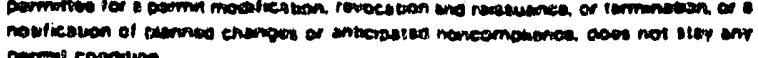

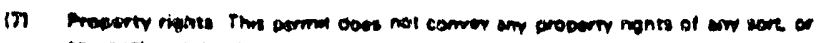

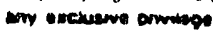

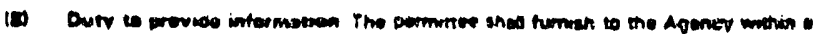

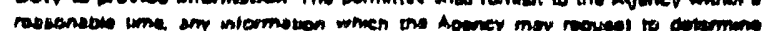

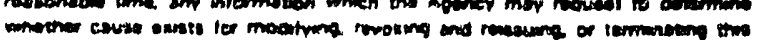

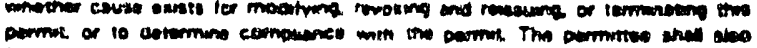

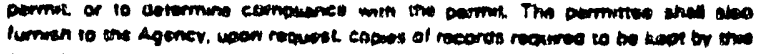
Derme

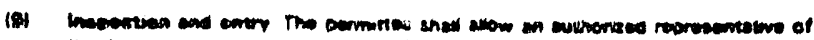

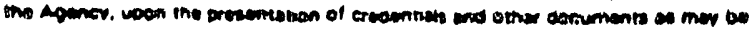
reoumes by tow. 10.

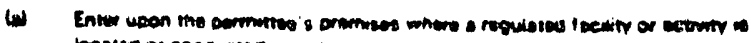

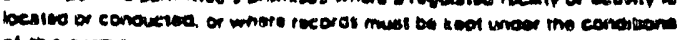
of the owmet.

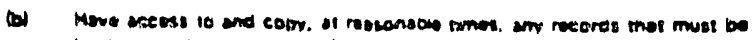
heot unoer ine conctions of this permet:

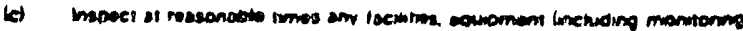

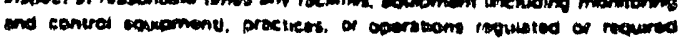

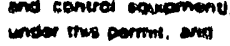

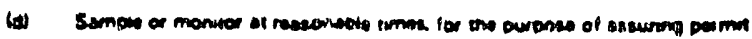

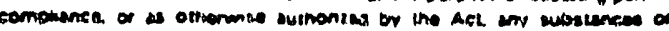

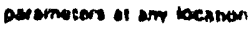

\section{Monstoring and recores}

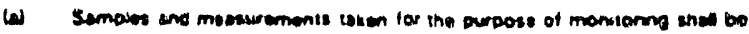

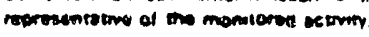

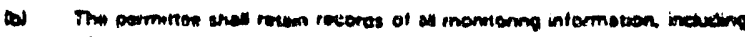

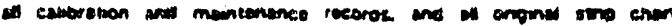

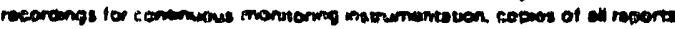

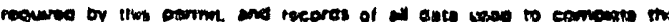

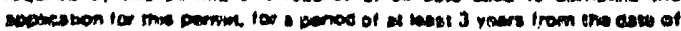

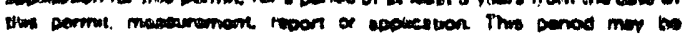

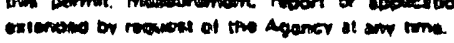

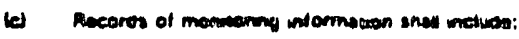

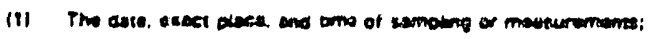

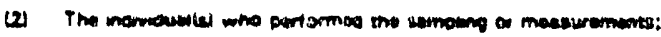

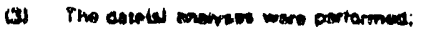

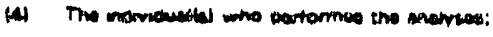

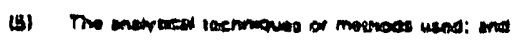

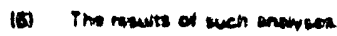

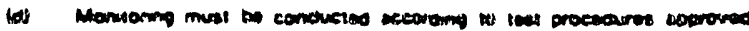

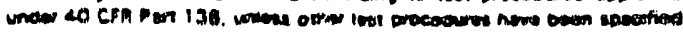

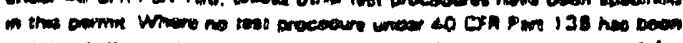

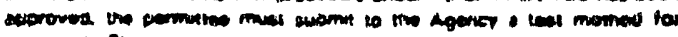

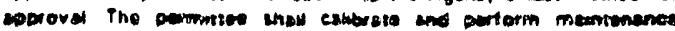

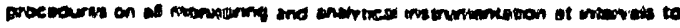

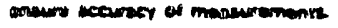

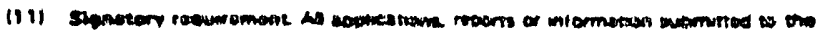

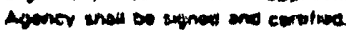

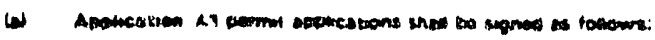

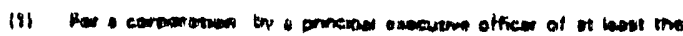

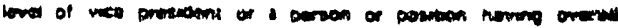

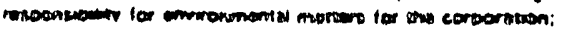

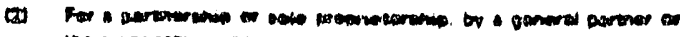

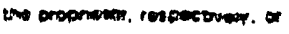

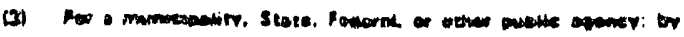

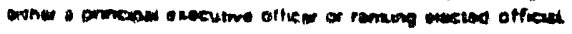

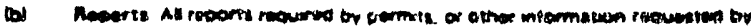

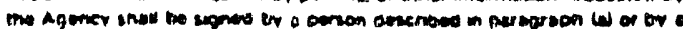

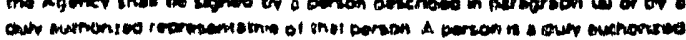

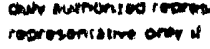

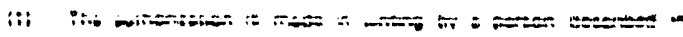

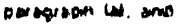

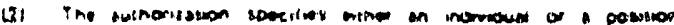

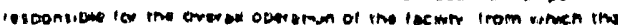

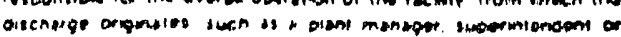

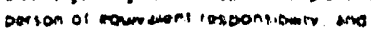

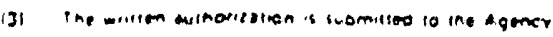




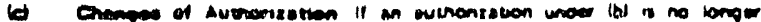

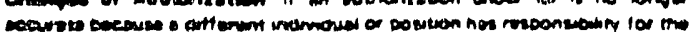

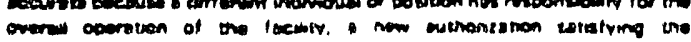

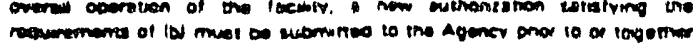

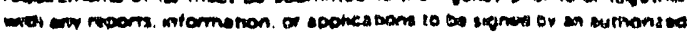
normonom

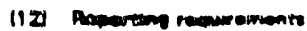

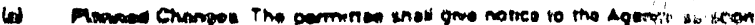

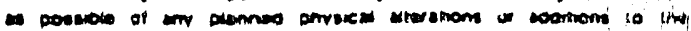
pumorted tecuers

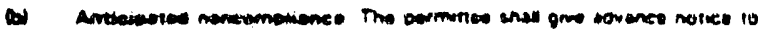

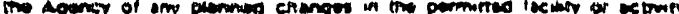

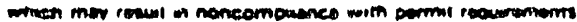

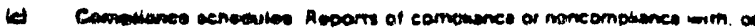

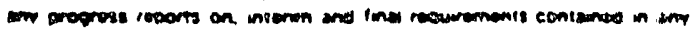

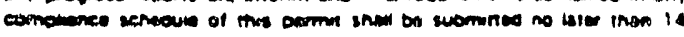

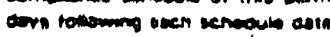

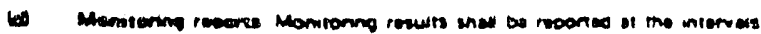

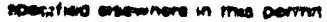

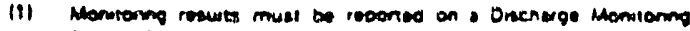
Rooun OMAI

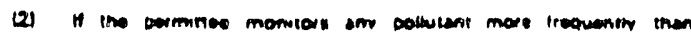

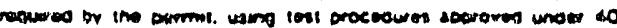

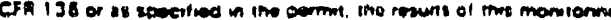

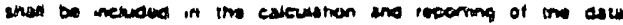
ueminteo on mo ONR

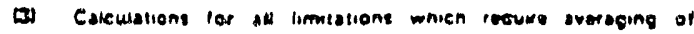

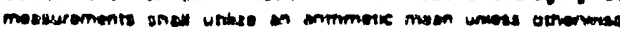

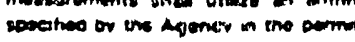

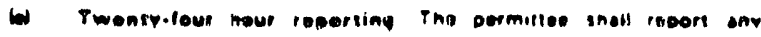

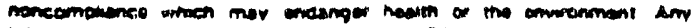

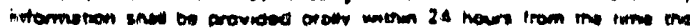

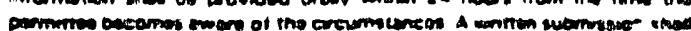

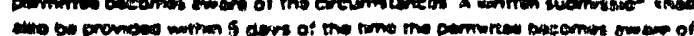

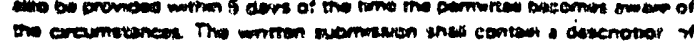

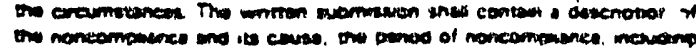

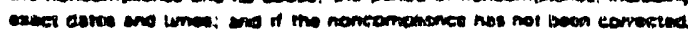

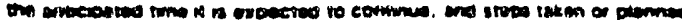

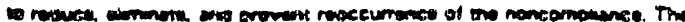

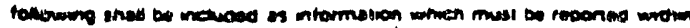
24 nours:

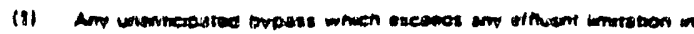
me permen.

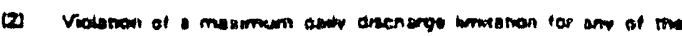

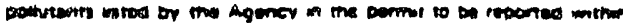
24 mours.

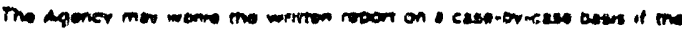

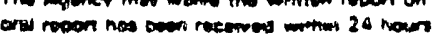

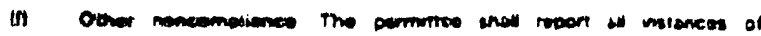

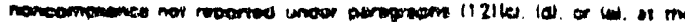

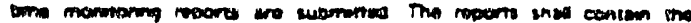

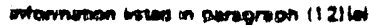

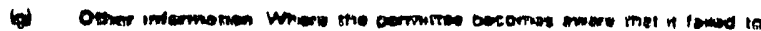

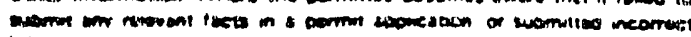

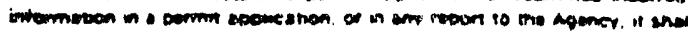

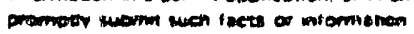

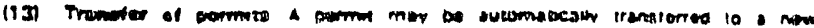
Troment

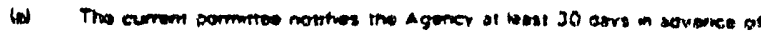

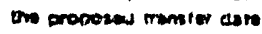

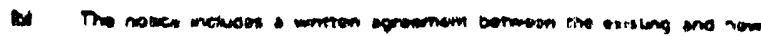

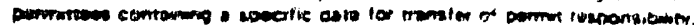

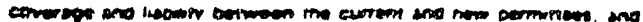

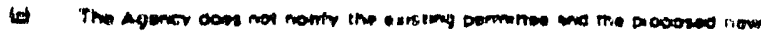

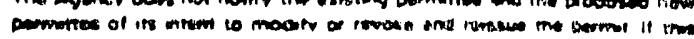

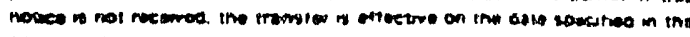
nomomen

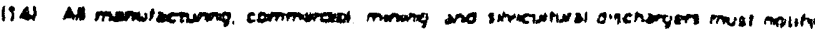

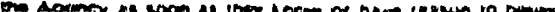

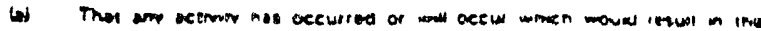

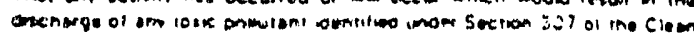

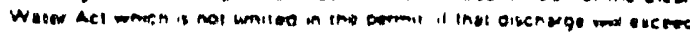

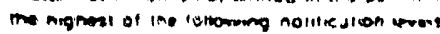

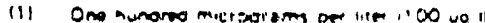

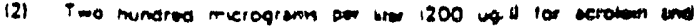

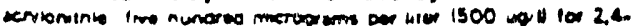

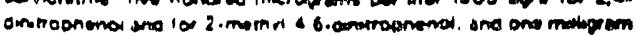
De lite 11 ma to immom

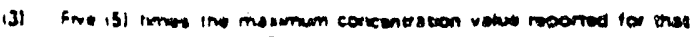

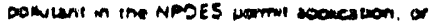

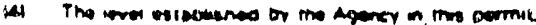

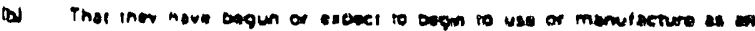

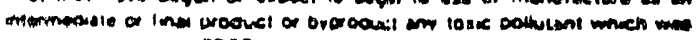

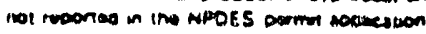

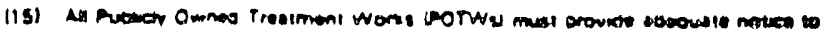
the Apencer of the lolloweming

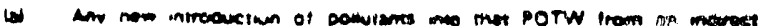

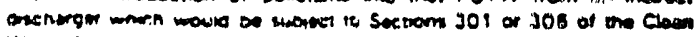

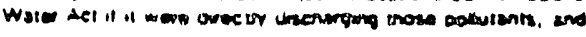

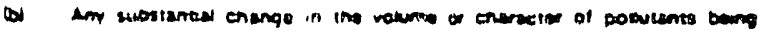

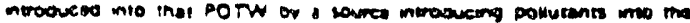

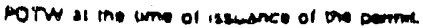

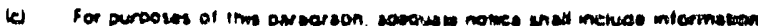

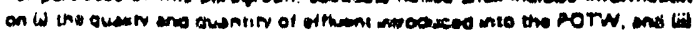

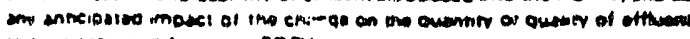
to oc aisenerged liam ine porw

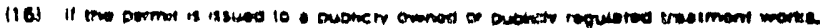

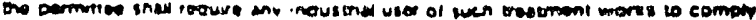

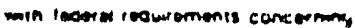

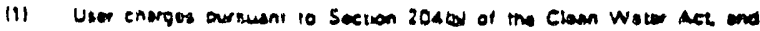

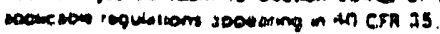

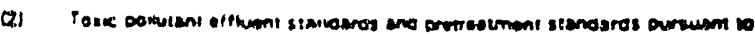
sactoon 307 of the Claen water atei. int

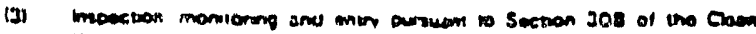
Woter Ast

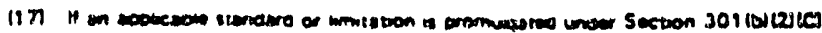

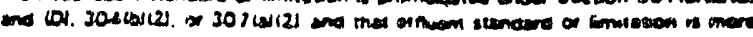

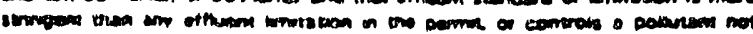

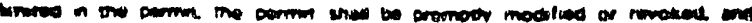

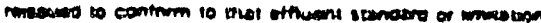

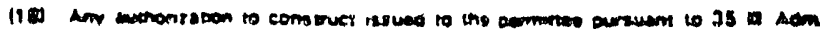

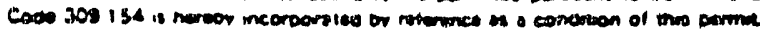

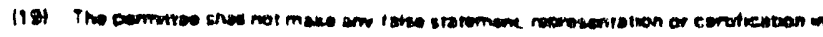

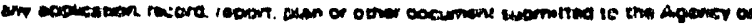

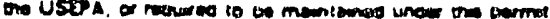

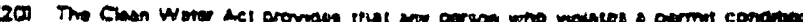

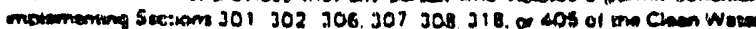

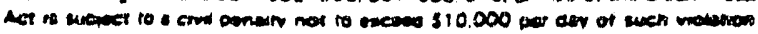

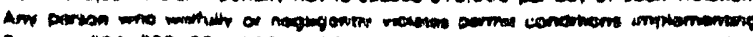

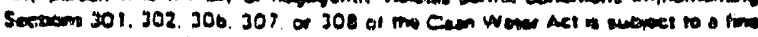

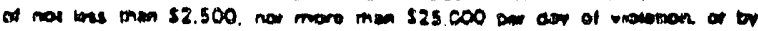

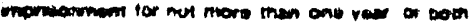

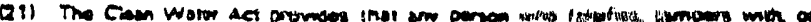

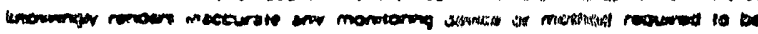

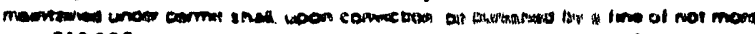

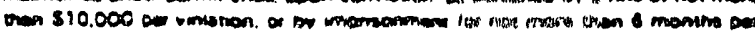
moxpmon of or com

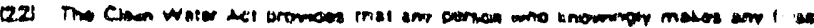

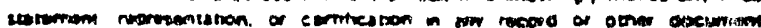

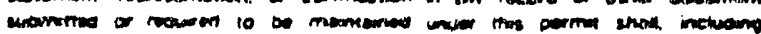

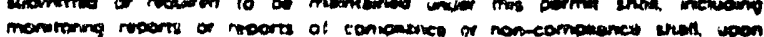

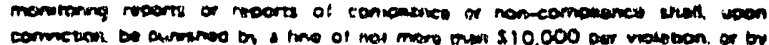

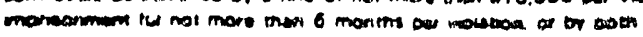

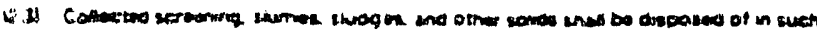

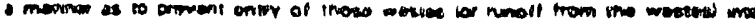

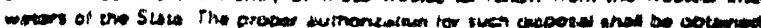

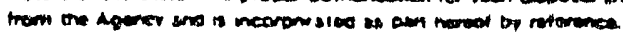

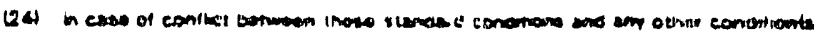

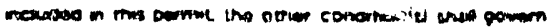

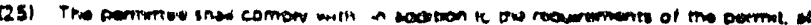

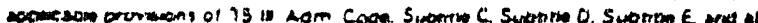

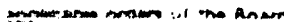

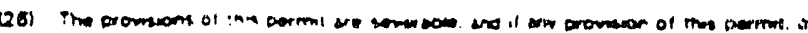

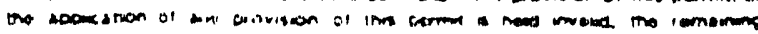

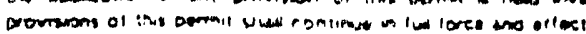

Ron $121.8 \mathrm{~A}$ 

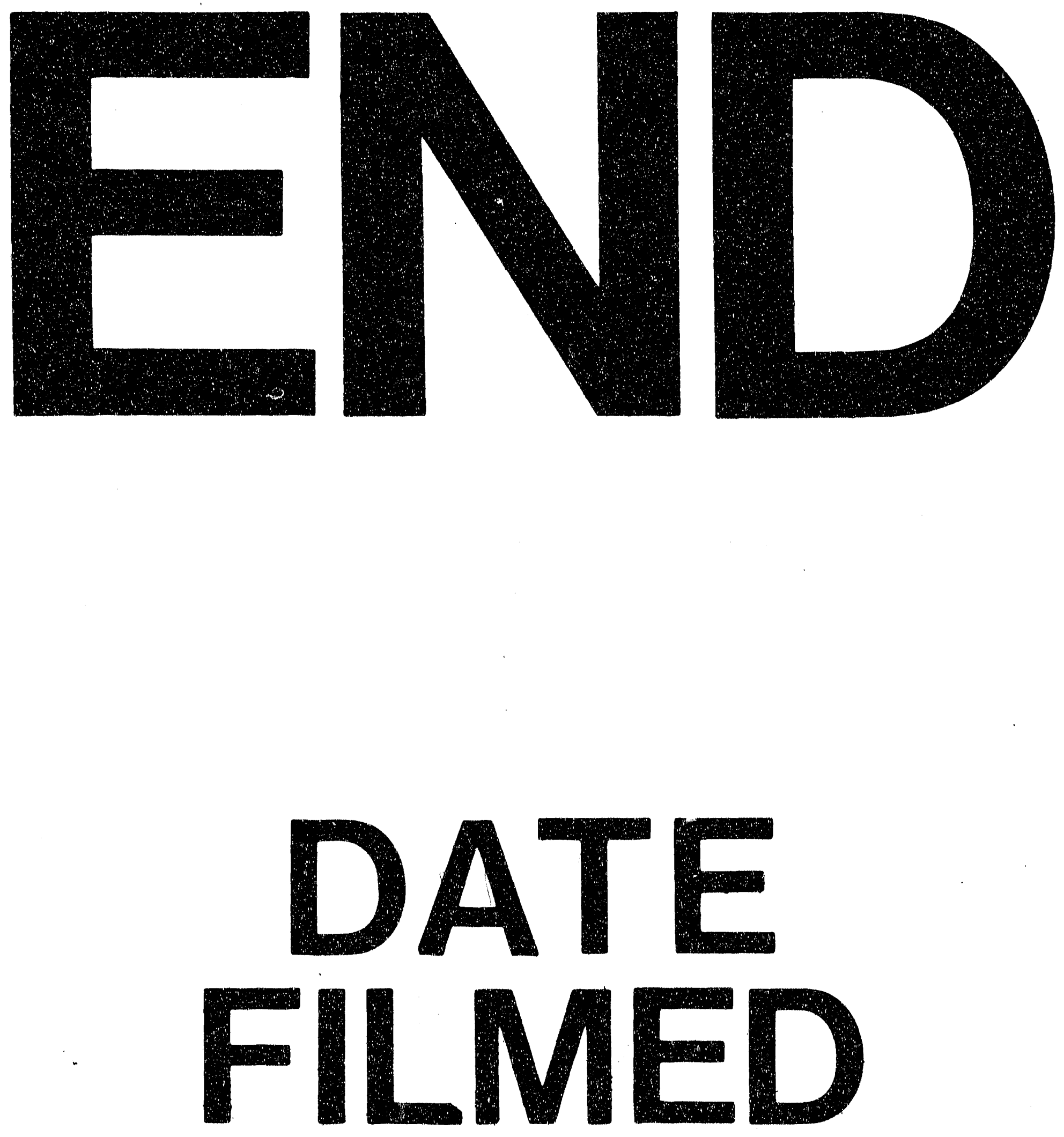

产

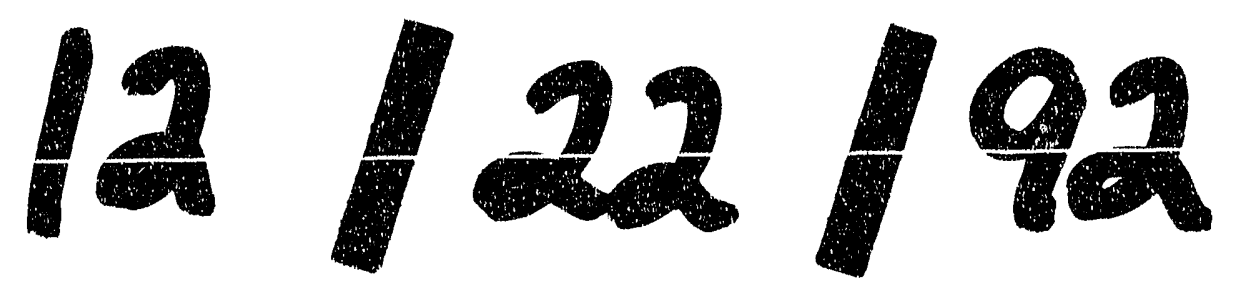




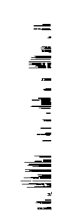

